\title{
INDEPENDENT SOURCE EXTRACTION APPLIED TO RADAR IMAGING
}

\author{
by \\ JEFFREY BRANDON HALL \\ Presented to the Faculty of the Graduate School of \\ The University of Texas at Arlington in Partial Fulfillment \\ of the Requirements \\ for the Degree of
} MASTER OF SCIENCE IN ELECTRICAL ENGINEERING THE UNIVERSITY OF TEXAS AT ARLINGTON August 2009 
Copyright $($ ) by Jeffrey Hall 2009

All Rights Reserved 


\section{ACKNOWLEDGEMENTS}

I would like to thank Dr. Tjuatja for kindling my interest in Synthetic Aperture Radar and Radar Imaging. His exuberance and principled guidance has led to my successful emersion into the topic at hand.

I would also like to thank the team at the Wave Scattering Research Center at the University of Texas at Arlington for their many discussions regarding this work. I would like to specially thank Mr. Suman Gunnala for all of his hard work in obtaining high quality measurement data and Mr. Luis Camacho for his enlightening conversations regarding this work.

Special thanks to Dr. Bredow and Dr. Aktosun for their participation in review of this work. Finally and most importantly, I would like to dedicate this work to my Dad who went to be with the Lord during its completion. I will always be grateful for his exemplification of hard work and perseverance which continues to motivate me.

July 10, 2009 


\title{
ABSTRACT \\ INDEPENDENT SOURCE EXTRACTION APPLIED \\ TO RADAR IMAGING
}

\author{
Jeffrey Brandon Hall, M.S. \\ The University of Texas at Arlington, 2009 \\ Supervising Professor: Saibun Tjuatja
}

Multiple scattering and random interactions among scattering elements and between the scatterers and the background adversely affect the radar image quality and target detection capability. In radar images formed using physical optics (PO) based techniques, multiple scattering and interactions appear as non-physical scattering centers. A method to enhance the performance of PO based radar imaging systems by extracting independent scattering centers is investigated in this thesis. Independent Component Analysis (ICA) is applied to returns of a radar system to extract independent scattering elements based on their non-Gaussianity. As an example, this method of target extraction is implemented in inverse synthetic aperture radar (ISAR) imaging of a variety of targets. Results of this study show that the application of this radar signal processing technique has allowed extraction of independent components which are related to scattering elements within the target. 


\section{TABLE OF CONTENTS}

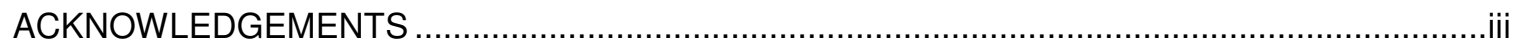

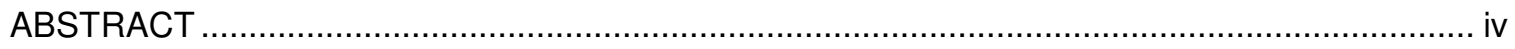

LIST OF ILLUSTRATIONS .........................................................................................

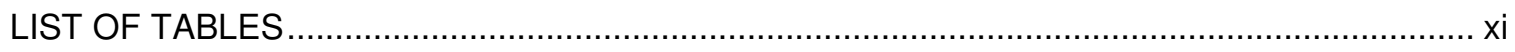

$\begin{array}{lll}\text { Chapter Page } & \text { Pas }\end{array}$

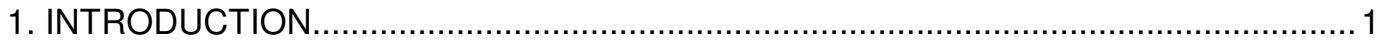

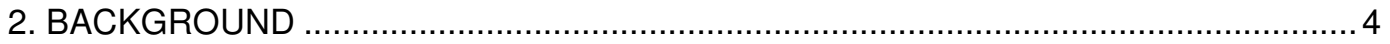

2.1 Inverse Synthetic Aperture Radar (ISAR) Background .................... 4

2.2 Radar Multipath Physical Development....................................... 8

2.3 Independent Component Analysis (ICA) Background ..................... 10

2.4 ICA Application to Radar Scattering …......................................... 13

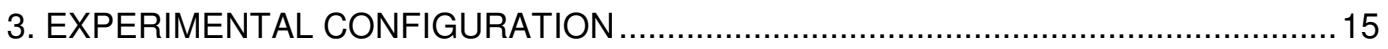

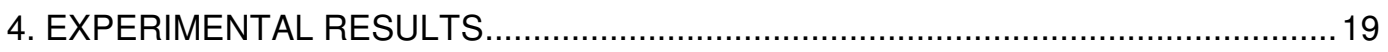

4.1 Orthogonalization Approach Parameter Selection......................................24

4.2 Whitening Matrix Dimension Selection .................................................. 42

4.3 Independent Component Quantity Parameter Selection .............................. 48

4.4 Nonlinear Estimator Parameter Selection............................................ 48

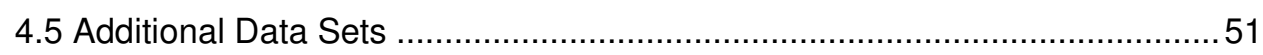

4.5.1 Offset 4" Sphere................................................................... 51

4.5.2 Four Inch Sphere and Six Inch Sphere: 8" Separation ...................54

4.5.3 Four Inch Sphere and Six Inch Sphere: 16" Separation................58

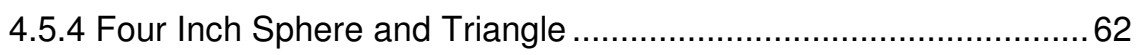

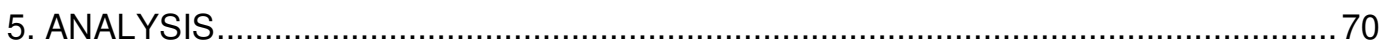




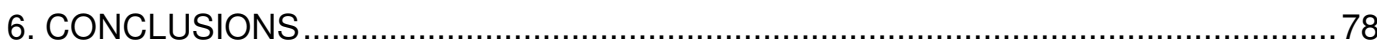

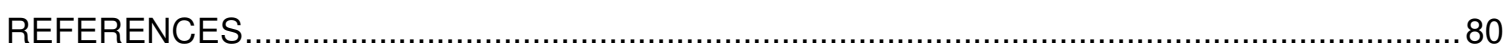

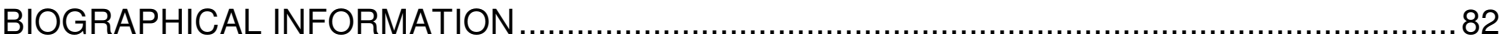




\section{LIST OF ILLUSTRATIONS}

Figure

Page

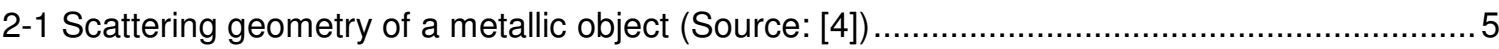

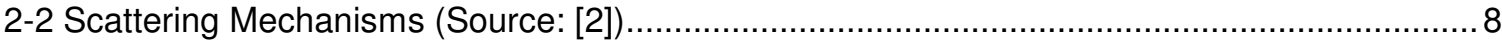

2-3 FastICA algorithm for estimating several ICs with deflationary orthogonalization

2-4 FastICA algorithm for estimating several ICs with symmetric orthogonalization

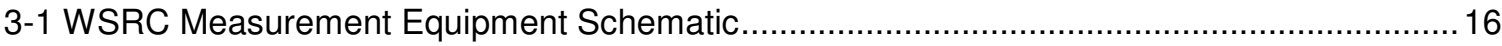

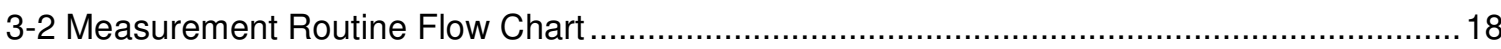

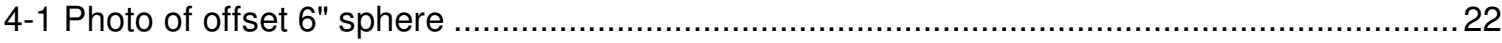

4-2 (a) ISAR image formed with raw scattering data

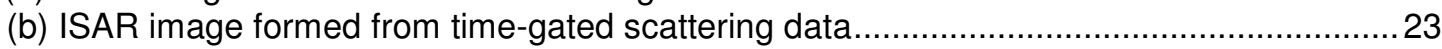

4-3 Difference between non-time gated image and time gated image ......................................24

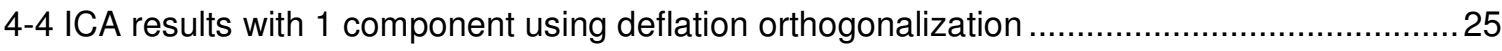

4-5 ICA results with 1 component using symmetric orthogonalization .....................................26

4-6 ICA results using deflation orthogonalization (a) component \#1

(b) component \#2

4-7 ICA results using symmetric orthogonalization (a) component \#1

(b) component \#2

4-8 ICA results using deflation orthogonalization (a) component \#1

(b) component \#2 (c) component \#3

4-9 ICA results using symmetric orthogonalization (a) component \#1

(b) component \#2 (c) component \#3

4-10 ICA results using deflation orthogonalization (a) component \#1

(b) component \#2 (c) component \#3 (d) component \#4

4-11 ICA results using symmetric orthogonalization (a) component \#1

(b) component \#2 (c) component \#3 (d) component \#4

4-12 ICA results using deflation orthogonalization (a) component \#1

(b) component \#2 (c) component \#3 (d) component \#4 (e) component \#5. 
4-13 ICA results using symmetric orthogonalization (a) component \#1

(b) component \#2 (c) component \#3 (d) component \#4 (e) component \#5

4-14 ICA results using deflation orthogonalization (a) component \#1

(b) component \#2 (c) component \#3 (d) component \#4 (e) component \#5

(f) component \#6

4-15 ICA results using symmetric orthogonalization (a) component \#1

(b) component \#2 (c) component \#3 (d) component \#4 (e) component \#5

(f) component \#6

4-16 ICA results using deflation orthogonalization (a) component \#1

(b) component \#2 (c) component \#3 (d) component \#4 (e) component \#5

(f) component \#6 (g) component \#7

4-17 ICA results using symmetric orthogonalization (a) component \#1

(b) component \#2 (c) component \#3 (d) component \#4 (e) component \#5

(f) component \#6 (g) component \#7

4-18 ICA results using deflation orthogonalization (a) component \#1

(b) component \#2 (c) component \#3 (d) component \#4 (e) component \#5

(f) component \#6 (g) component \#7 (h) component \#8...

4-19 ICA results using symmetric orthogonalization (a) component \#1

(b) component \#2 (c) component \#3 (d) component \#4 (e) component \#5

(f) component \#6 (g) component \#7 (h) component \#8

4-20 ICA results using deflation orthogonalization (a) component \#1

(b) component \#2 (c) component \#3 (d) component \#4 (e) component \#5

(f) component \#6 (g) component \#7 (h) component \#8 (i) component \#9.

4-21 ICA results using symmetric orthogonalization (a) component \#1

(b) component \#2 (c) component \#3 (d) component \#4 (e) component \#5

(f) component \#6 (g) component \#7 (h) component \#8 (i) component \#9. 42

4-22 Eigenvalue retention metric for offset 6" sphere 44

4-23 ICA results with 2 ICs and 2 EVs (a) component \#1 (b) component \#2

4-24 ICA results with 9 ICs and 12 EVs (a) component \#1

(b) component \#2 (c) component \#3 (d) component \#4 (e) component \#5

(f) component \#6 (g) component \#7 (h) component \#8 (i) component \#9. 46

4-25 ICA results with $9 \mathrm{ICs}$ and $15 \mathrm{EVs}$ (a) component \#1

(b) component \#2 (c) component \#3 (d) component \#4 (e) component \#5

(f) component \#6 (g) component \#7 (h) component \#8 (i) component \#9.

4-26 Nonlinear estimator comparison (a) gaus: component \#1

(b) gaus: component \#2 (c) gaus: component \#3, (d) pow3: component \#1

(e) pow3: component \#2 (f) pow3: component \#3 (g) skew: component \#1

(h) skew: component \#2 (i) skew: component \#3 (j) tanh: component \#1

(k) tanh: component \#2 (I) tanh: component \#3 .50

4-27 Photo of offset 4" sphere 
4-28 (a) ISAR image formed with raw scattering data

(b) ISAR image formed from time-gated scattering data ................................................... 52

4-29 Eigenvalue retention metric for offset 4" sphere ................................................................53

4-30 ICA results with 8 ICs and 8 EVs (a) component \#1

(b) component \#2 (c) component \#3 (d) component \#4 (e) component \#5

(f) component \#6 (g) component \#7 (h) component \#8 …..................................................... 54

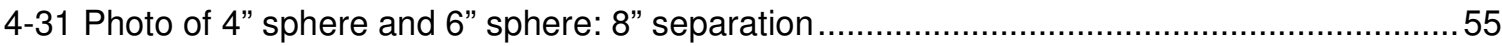

4-32 (a) ISAR image formed with raw scattering data

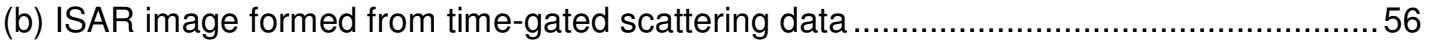

4-33 EV retention metric for 4" sphere and 6" sphere @ 8" separation ........................................57

4-34 ICA results with $8 \mathrm{ICs}$ and $8 \mathrm{EVs}$ (a) component \#1

(b) component \#2 (c) component \#3 (d) component \#4 (e) component \#5

(f) component \#6 (g) component \#7 (h) component \#8 ...

4-35 Photo of 4" sphere and 6" sphere: 16" separation

4-36 (a) ISAR image formed with raw scattering data

(b) ISAR image formed from time-gated scattering data

4-37 EV retention metric for 4" sphere and 6" sphere @ 16" separation

4-38 ICA results with $11 \mathrm{ICs}$ and $11 \mathrm{EVs}$ (a) component \#1

(b) component \#2 (c) component \#3 (d) component \#4 (e) component \#5

(f) component \#6 (g) component \#7 (h) component \#8 (i) component \#9

(j) component \#10 (k) component \#11

4-39 Photo of 4" sphere and triangle

4-40 (a) ISAR image formed with raw scattering data

(b) ISAR image formed from time-gated scattering data 64

4-41 EV retention metric for 4" sphere and triangle

4-42 ICA results with 6 ICs and 6 EVs (a) gaus: component \#1

(b) gaus: component \#2 (c) gaus: component \#3 (d) gaus: component \#4

(e) gaus: component \#5 (f) gaus: component \#6

4-43 ICA results with 6 ICs and 6 EVs (a) pow3: component \#1

(b) pow3: component \#2 (c) pow3: component \#3 (d) pow3: component \#4

(e) pow3: component \#5 (f) pow3: component \#6

4-44 ICA results with 6 ICs and 6 EVs (a) skew: component \#1

(b) skew: component \#2 (c) skew: component \#3 (d) skew: component \#4

(e) skew: component \#5 (f) skew: component \#6

4-45 ICA results with 6 ICs and 6 EVs (a) tanh: component \#1

(b) tanh: component \#2 (c) tanh: component \#3 (d) tanh: component \#4

(e) tanh: component \#5 (f) tanh: component \#6 
5-1 Independent components for 6" sphere (a) component \#1

(b) component \#2 (c) component \#3 (d) component \#4 (e) component \#5

(f) component \#6 (g) component \#7 (h) component \#8 (i) component \#9.

5-2 Independent components for 4" sphere (a) component \#1

(b) component \#2 (c) component \#3 (d) component \#4 (e) component \#5

(f) component \#6 (g) component \#7 (h) component \#8.

5-3 Independent components for 6" and 4" spheres with 8" spacing

(a) component \#1 (b) component \#2 (c) component \#3 (d) component \#4

(e) component \#5 (f) component \#6 (g) component \#7 (h) component \#8 .73

5-4 Independent components for 6" and 4" spheres with 16" spacing

(a) component \#1 (b) component \#2 (c) component \#3 (d) component \#4

(e) component \#5 (f) component \#6 (g) component \#7 (h) component \#8

(i) component \#9 (j) component \#10 (k) component \#11 .75

5-5 Independent components for 4" spheres and triangle

(a) tanh: component \#1 (b) tanh: component \#2 (c) tanh: component \#3

(d) tanh: component \#4 (e) tanh: component \#5 (f) tanh: component \#6 


\section{LIST OF TABLES}

Table

3-1 Experimental Measurement Parameters .

4-1 FastICA Parameter Selections
Page

17

20 


\section{CHAPTER 1}

INTRODUCTION

Radio Detection and Ranging (RADAR) is an all weather, day or night remote sensing modality [1]. The enabler, for the robustness of radar, is the use of self-generated emissions to probe targets rather than relying on the internal emissions of a target or scattered emissions from some other source outside the radar's control. The active sensing aspect of radar remote sensing affords the modality with the advantage of sensing at great ranges and the ability to sense through clouds, rain or dust. Because of its robustness, the use of radar has found widespread application [2]. Along with its robustness the radar modality suffers some shortcomings relative to other sensing regimes. One of the major disadvantages of sensing via radar is the proclivity for interference due to interactions of several closely spaced scatters.

The phenomenon of multi-scatterer interaction arises from the multiple reflection paths presented by a complex geometry of closely spaced scatters. A radar return which is a result of multi-bounce or a traveling wave is perceived by the receiving system as a physical target. In actuality the target elements generating the multiple bounce or traveling wave return may not even be at the same angle relative to the interrogating system as the perceived target. The multiple bounce and traveling wave returns will indefinitely be perceived at a different range then the physical targets. This phenomenological rule is due to the additional path length the radar signal is required to traverse due to the multiple bounces. This scattering interference presents a number of issues in interpreting radar returns. One of the issues specifically related to Inverse Synthetic Aperture Radar (ISAR) will be addressed in this thesis. The issue is that false scattering centers complicate the discernment of the true scattering centers. A scattering center may be 
defined as the phase center of a portion of the target. The phase center for an object is the point at which the energy being returned to the sensing system appears to emanate. It is the point at which the reflections from nearby scattering elements coherently combine at the receiving antenna.

The ability of radar to discern closely spaced scatterers is limited by the resolution of the interrogating radar system as well as the radar cross-section (RCS) of the separate scatters. The composite RCS, created by the interaction of closely spaced scatterers, can present the radar system with a virtual target which competes for detection sensitivity with the actual targets. The composite RCS can be physically described as the system's response to a multipath return. In certain geometrical circumstances, a large target return may be perceived in a spatial area where a physical target is absent. This is due to the temporal delay induced by an additional path length produced by the random multiple-scattering geometry [3][4]. For geometrically variant targets, the challenge of consistently distinguishing a direct reflection "real" return from a multipath "ghost" return is exacerbated [4][5]. This paper attempts to present a method for overcoming the misperception of an electromagnetic sensor system, through the use of the statistics of the target return, to distinguish between returns generated by multiple scattering elements when presented with this physical circumstance. This method involves the application of a well known statistical signal processing technique, Independent Component Analysis (ICA), to electromagnetic scattering data. The results of this study show that it is possible to separate the independent components which have some relationship to the physical scattering mechanisms of a target.

To enable ready visualization of the effect of this approach to scattering center separation, an Inverse Synthetic Aperture Radar (ISAR) system is used. For this study, measurements were conducted inside the anechoic chamber at the University of Texas at Arlington Wave Scattering Research Center.

This thesis is organized in the following manner. Chapter 2 will introduce the fundamental behind ISAR and geometric optics based radar imaging. Chapter 2 will also outline the physics behind multiple scattering phenomena in radar sensing. Chapter 3 will provide an overview of the concept of ICA and the mathematical preliminaries relative to its application to ISAR data. Chapter 4 introduces the reader to the measurement system at the Wave Scattering Research 
Center at UTA on which all of the measurements used for this thesis were taken. Chapter 5 presents the results of the application of ICA to the ISAR returns from multiple target sets.

Chapter six concludes the paper with a summary of the work and inference of the analysis presented in this thesis. 


\section{CHAPTER 2}

BACKGROUND

The first section of this chapter is directed at introducing the concept of inverse synthetic aperture radar and the physics associated with geometrics optics based radar imaging techniques. Of particular interest are the approximations necessary to enable image generation through the use of a 2-dimensional Fourier transform and the implications of these approximations when faced with real-world targets which violate them.

The second half of the chapter is dedicated to development of the concept of radar multipath. This section establishes a distinction between the multiple scattering elements of a single target. A graphic to illustrate the multitude of scattering elements which can be present on a single target is presented. Finally a mathematical model for superposition of the multipath returns in a radar measurement is given.

\subsection{Inverse Synthetic Aperture Radar (ISAR) Background}

Inverse synthetic aperture processing allows a human access to the space domain representation of a target that one is accustomed to when using modalities of higher frequency such as photographs. To coerce a target representation into this format it is necessary to provide adequate resolution to capture the target detail. Radar systems typically have good resolution in the down range direction but poor resolution in the crossrange direction. This lack of resolution in the crossrange is due to the limited available aperture size for wavelengths within the microwave region. In order to enhance the crossrange resolution a post detection processing scheme known as aperture synthesis is performed. This technique applies coherent processing of radar returns from a diversity of look angles. This process is akin to aperture processing which is performed in 
a predetection domain on antenna arrays. The ability to perform postprocessing of the detected returns allows for the use of a simple antenna system which can achieve high crossrange resolution.

The geometry of a generic scattering geometry is shown in Figure 2-1. This geometry depicts a most general case for any relative location of the transmitting and receiving antennas. In this thesis we will focus on a pseudo-monostatic scenario in which the transmitting and receiving antennas are located in very close proximity to one another. The distinction between a monostatic and a pseudo-monostatic arrangement lies in the number of physical antennas used. For a monostatic arrangement, a single antenna is used for transmit and receive functions and for the pseudo-monostatic case these functions are performed by separate antennas. The antennas in the latter case are arranged such that target returns perceived by the receiving antenna very closely approximate the range and angle which would be sensed by a single antenna. This arrangement allows for the exclusion of microwave hardware associated with switching a single channel between transmit and receive states in a timely manner.

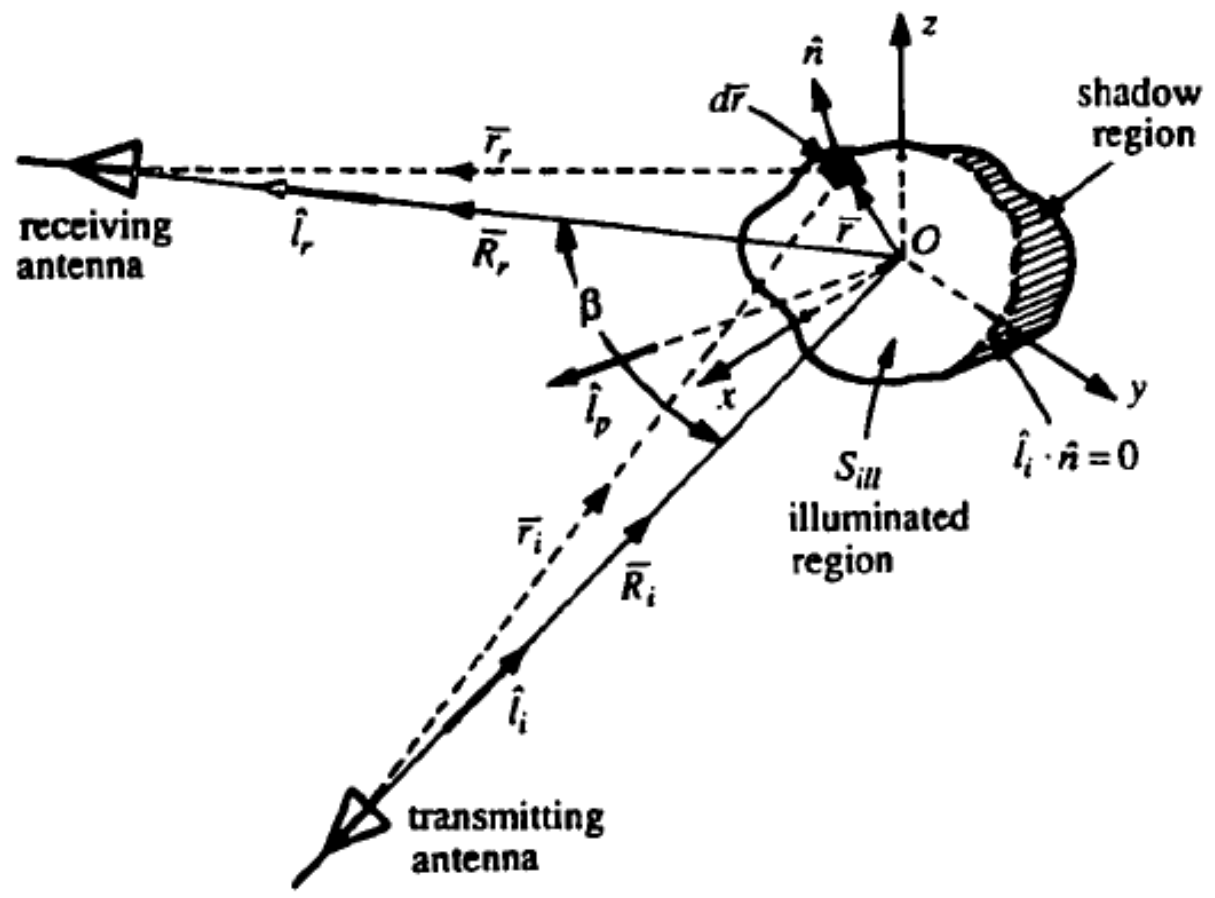

Figure 2-1 Scattering geometry of a metallic object (Source: [4]) 
Using the geometry outlines in Figure 2-1, it is helpful to define the scattering problem in terms of incident and scattered waves. This definition yields

$$
\begin{aligned}
& \bar{E}^{\mathrm{t}}=\bar{E}^{\mathrm{i}}+\bar{E}^{\mathrm{s}} \\
& \bar{H}^{\mathrm{t}}=\bar{H}^{\mathrm{i}}+\bar{H}^{\mathrm{s}}
\end{aligned}
$$

Images formed from radar scattering data are done so based on a number of assumptions which allow the Stratton-Chu integral equations to be simplified [6]. The complete Stratton-Chu integral equations for the scattered fields are given by

$$
\begin{aligned}
& \bar{E}^{\mathrm{s}}\left(\bar{R}_{r}\right)=\frac{1}{4 \pi} \int\left[\left(\hat{n} \times \bar{E}^{t}\right) \times \nabla \Psi_{0}-j \omega \mu\left(\hat{n} \times \bar{H}^{t}\right) \Psi_{0}+\left(\hat{n} \cdot \bar{E}^{t}\right) \nabla \Psi_{0}\right] d s \\
& \bar{H}^{\mathrm{s}}\left(\bar{R}_{r}\right)=\frac{1}{4 \pi} \int\left[\left(\hat{n} \times \bar{H}^{t}\right) \times \nabla \Psi_{0}-j \omega \varepsilon\left(\hat{n} \times \bar{E}^{t}\right) \Psi_{0}+\left(\hat{n} \cdot \bar{H}^{t}\right) \nabla \Psi_{0}\right] d s
\end{aligned}
$$

where the integration range is defined by the limits of the objects surface.

The first assumption made is in regard to the target media. If we assume that the target is a perfect conductor we know $\hat{n} \times \bar{E}^{t}=\hat{n} \cdot \bar{H}^{t}=0$ on the object surface. This allows equations (2-3) and (2-4) to be simplified to

$$
\begin{gathered}
\bar{E}^{\mathrm{s}}\left(\bar{R}_{r}\right)=\frac{1}{4 \pi} \int\left[-j \omega \mu\left(\hat{n} \times \bar{H}^{t}\right) \Psi_{0}+\left(\hat{n} \cdot \bar{E}^{t}\right) \nabla \Psi_{0}\right] d s \\
\bar{H}^{\mathrm{s}}\left(\bar{R}_{r}\right)=\frac{1}{4 \pi} \int\left[\left(\hat{n} \times \bar{H}^{t}\right) \times \nabla \Psi_{0}\right] d s
\end{gathered}
$$

The next few assumptions which allow the Stratton-Chu integral equations to be simplified are collectively known as the physical optics (PO) approximation. There are three key assumptions that make up the PO approximation. The first assumption is that the fields within the shadowed region, as shown in Figure 2-1, are set to zero. The second assumption is that the scattered fields are being observed in the far-field region. This assumption allows for simplification of the free space Green's function to

$$
\Psi_{0}=\frac{e^{-j k R_{r}} e^{j k \hat{l}_{r} \cdot \bar{r}}}{R_{r}}
$$

where $\hat{l}_{r}$ represents the direction of the between the target and the receiving antenna. 
The last assumption is known as the tangent plane approximation which requires that the radii of curvature at all points on the target are much greater than a wavelength of the interrogating signal. This approximation allows one to make use of Snell's law for a perfectly conducting plane.

$$
\hat{n} \times \bar{E}^{i}=0 ; \quad \hat{n} \times \bar{H}^{i}=2 \hat{n} \times \bar{H}^{i}
$$

Using the second assumption applied to the incident field portion of problem, we will now define these fields through their approximation as a local plane wave.

$$
\begin{gathered}
\bar{E}^{i} \approx \bar{E} \frac{e^{-j k R_{i}} e^{-j k \hat{l}_{i} \cdot \bar{r}}}{R_{i}} \\
\bar{H}^{i} \approx \bar{H} \frac{e^{-j k R_{i}} e^{-j k \hat{l}_{i} \cdot \bar{r}}}{R_{i}}
\end{gathered}
$$

Upon application of the physical optics assumptions, we can now define the scattered fields in terms of the incident fields and Equations (2-5) and (2-6) become

$$
\begin{gathered}
\bar{E}^{\mathrm{s}}\left(\bar{R}_{r}\right)=\frac{-j \omega \mu}{2 \pi R_{r}} e^{-j k R_{r}} \iint\left[\left(\left(\hat{n} \times \bar{H}^{i}\right)-\left(\hat{l}_{r} \cdot \hat{n} \times \bar{H}^{i}\right)\right) \hat{l}_{r}\right] e^{j k \hat{l}_{r} \cdot \bar{r}^{\prime}} d s_{i l l} \\
\bar{H}^{\mathrm{s}}\left(\bar{R}_{r}\right)=\frac{j k}{2 \pi R_{r}} e^{-j k R_{r}} \int\left[\left(\hat{n} \times \bar{H}^{i}\right) \times \hat{l}_{r}\right] e^{j k \hat{l}_{r} \cdot \bar{r}} d s_{i l l}
\end{gathered}
$$

In (2-11) and (2-12) the surface upon which the integral operates is the illuminated surface of the target object. The surface is defined as the surface of the target which is facing the transmitting antenna and bounded by $\hat{n} \cdot \hat{l}_{r}=0$. In the case of the pseudo-monostatic arrangement this can also be approximated as the surface which is also facing the receiving antenna. This reveals the requirement for the target to be convex to assure a connected surface for the integration.

ISAR image formation is based on the Fourier-transform relationship between scattered fields in k-space and an object's scattering function [7]. Bojarski's identity gives the Fourier transform relationship between scattered field, $\bar{E}_{s}\left(k_{x}, k_{y}\right)$ and the object reflectivity function, $\Gamma(x, y)$ which is related to surface shape of the scattering object [8]. The scattered electric field is a function of a variety of parameters. Some of these parameters, such as frequency and polarization, are set by the radar system. Others, such as the target shape and the material of the object being interrogated, are generally unknown. The scattered field can be measured directly in order to estimate the target properties. 


$$
\overline{\mathrm{E}}_{\mathrm{s}}\left(k_{x}, k_{y}\right)=\int_{-\infty}^{\infty} \int_{-\infty}^{\infty} \Gamma(x, y) \exp \left(-j 2 \pi\left(k_{x} x+k_{y} y\right)\right) d x d y
$$

The object reflectivity function, given by inverse Fourier transform of the measured scattered field, gives the user of the imaging system a sense of the object's geometrical parameters.

$$
\bar{\Gamma}(x, y)=\int_{-\infty}^{\infty} \int_{-\infty}^{\infty} \overline{\mathrm{E}}_{\mathrm{s}}\left(k_{x}, k_{y}\right) \exp \left(j 2 \pi\left(k_{x} x+k_{y} y\right)\right) d k_{x} d k_{y}
$$

The mathematical relationship, between the scattered field and an objects reflectivity function, allows one to construct an image of the re-radiating surface of the target through sensing of the scattered field. Through measurement of the scattered field and digital signal processing over a 2 dimensional space-time set we are able to project an image of the object(s) on to a plane. This allows for image creation using scattered microwave fields.

\subsection{Radar Multipath Physical Development}

A radar return from a target observation can be considered to be a mixture of returns from the target due to the direct reflection of the interrogating radar wave as well as returns presented by scattering from the various scattering mechanisms. Figure 2-2 illustrates some of the various mechanisms of scattering that can result in reflected energy being received by the sensing system.

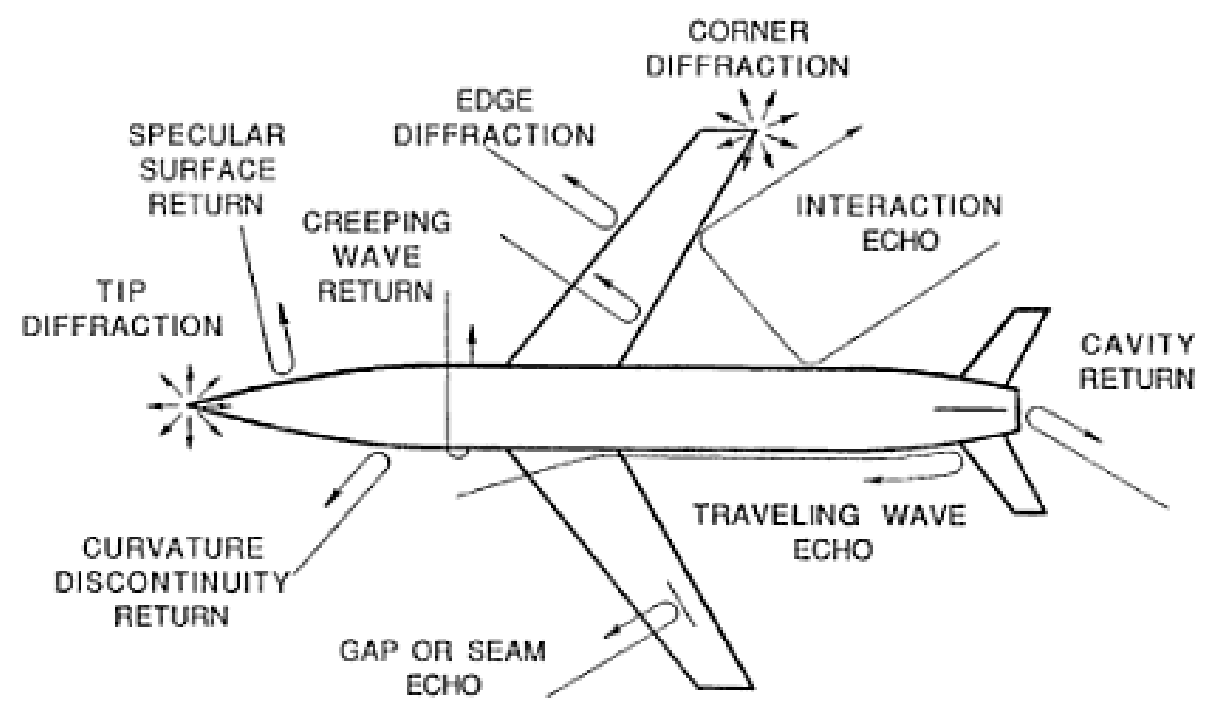

Figure 2-2 Scattering Mechanisms (Source: [2]) 
The first order approximation of the scattered field from a complex object can be expressed as the linear superposition of fields scattered from the diversity of scattering mechanisms present on the target [9]. For the purpose of this thesis, the received mixture can be described by

$$
\begin{aligned}
& x_{1}=a_{11} s_{1}+a_{12} s_{2}+a_{13} s_{3}+\cdots \\
& x_{2}=a_{21} s_{1}+a_{22} s_{2}+a_{23} s_{3}+\cdots \\
& x_{3}=a_{31} s_{1}+a_{32} s_{2}+a_{33} s_{3}+\cdots
\end{aligned}
$$

To generalize the equations shown in (2-15), we will define the subscripts in the following manner: $a_{i j}, s_{j}$ and $x_{i}$. In this general notation, the coefficients $a_{i j}$ represent the mixing coefficient for a particular scatterer $s_{j}$ at a given look angle which corresponds to the index, $i$. The $x_{i}$ values are the radar measurements for a look angle index, $i$. Each $s_{j}$ corresponds to an underlying component in the mixture.

For the first look angle $a_{11}$ is the response coefficient of one scattering element of the target scene, $s_{1}$ and $a_{12}$ is the response coefficient of the second scattering element, $s_{2}$ and so forth. The transfer functions $a_{i j}$ are dependent on parameters such as target geometry, frequency, material and polarization. In radar remote sensing of a single target of interest, ideally there is but a single source of returning signal energy; however, for non-trivial targets in a cluttered environment a plethora of scattering "sources" may exist [10]. The scattering sources are represented in the above formulation by $s_{1}, s_{2}$ and $s_{3}$. The output of the mixing function, $x_{1}, x_{2}$, etc., can be considered an observation of the target response at a look angle index 1, look angle index 2, etc.

This mixture of signal sources presents the radar receiver with a number of undesired target responses, due to the multiple scattering sources, along with the desired target. Rigorous mathematical development of the multipath scattering phenomenon is given in [3] and [5]. It is the objective of this thesis to assess the viability of the separation the scattering sources based on the statistics of their returns. 


\subsection{Independent Component Analysis (ICA) Background}

The process of recovering independent sources, given only sensor observations which are unknown linear mixtures of the unobserved source signals, belongs to a category of methods known as blind source separation (BSS). In general these methods are used to extract information from mixtures of data for which no a priori knowledge of the nature of the data sources is necessary and only the outputs of the system are observable [11][12]. Blind source separation methods have been applied across a wide range of applications. If applied in the proper framework, the affectivity of these techniques is insensitive to the type of data to which they are applied. Applications ranging from analysis of stock market data to brain waves have found utility in BSS methods.

Independent Component Analysis (ICA) is a well known method for revealing hidden factors embedded in sets of random variables [13]. ICA uses the independence of elements of a mixture to extract valuable information from the mixture. ICA is unique within the realm of BSS methods in that the primary criterion for source separation is the independence of the source data elements of the data mixture [14]. A well known second-order BSS methodology is that of Principal Component Analysis (PCA). PCA is capable of producing separation based on full statistical independence of the elements of a mixture only if the elements are of Gaussian distribution [15]. First order scattered signals received by a radar system are generally nonGaussian and therefore PCA is not a well suited solution to the problem of separation of independent scattering centers in radar data [16]. To separate independent non-Gaussian sources, it is necessary to look to statistical signal processing methods based on higher order statistics (HOS) [15]. ICA is a method which belongs to the blind source separation (BSS) class of mathematical techniques and the FastICA algorithm is a particular implementation of the ICA method [13]. There are a number of similar ICA algorithms available [17]. Within this study we have employed the use of the FastICA algorithm. The FastlCA algorithm was chosen based on its ready availability and well documented code. Another motivating factor for the use of the FastICA algorithm was the availability of a text [14] to supplement the understanding of the algorithm function.

For the ICA formulation, we will consider the data generative model: 


$$
x=A s
$$

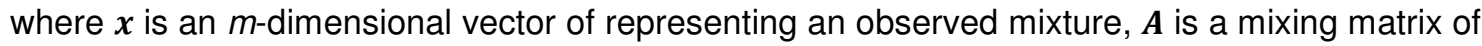
dimension $m$ by $n$ and $s$ is an $n$-dimensional vector of independent components.

The FastICA algorithm employs the information theoretic measure of mutual information to determine the transformation matrix which yields the most independent set of components. By definition the minimization of mutual information maximizes the independence of the components. The algorithm uses negentropy as a cost function. Mutual information can be expressed in terms of negentropy using:

$$
I\left(y_{1}, y_{2}, \ldots, y_{n}\right)=J(\boldsymbol{y})-\sum_{i} J\left(y_{i}\right)
$$

where $I$ is the mutual information for each of the elements of the vector $y$ and the function $J$ represents the negentropy. Negentropy is defined as:

$$
J(y)=H\left(y_{\text {gauss }}\right)-H(y)
$$

The maximization of negentropy results in the minimization of mutual information between the components of the mixture. The classical method of approximation is through the use of higher-order cumulants [14]. This method of approximation usually leads to the use of kurtosis.

$$
J(y)=\frac{1}{12} E\left\{y^{3}\right\}^{2}+\frac{1}{48} \operatorname{kurt}(y)^{2}
$$

However is can be shown that kurtosis is not robust measure of non-gaussianity due to its sensitivity to outliers. A modification to the use of kurtosis is to generalize the higher-order cumulants approximation using expectations of general non-quadratic functions, $G$.

$$
J(y) \propto[E\{G(y)\}-E\{G(v)\}]^{2}
$$

where $G$ is some non-quadratic function and $v$ is a Gaussian variable of zero mean and unit variance. The variable $y$ is assumed to also have zero mean and unit variance. Proper choice of $G$, such that growth is not too fast, yields better estimation of negentropy. Two examples of proven robust estimators are given in (2-21) and (2-22). 


$$
\begin{aligned}
& G_{1}(y)=\frac{1}{a_{1}} \log \cosh a_{1} y \\
& G_{2}(y)=-\exp \left(\frac{-y^{2}}{2}\right)
\end{aligned}
$$

where $1 \leq a_{1} \leq 2$ is some suitable constant usually taken as 1 .

A practical method for approximating negentropy is the use of a gradient algorithm. The algorithm for the gradient maximization of negentropy with respect to $\mathbf{w}$ can be derived from (2-20). It is necessary to include a normalization term $E\left\{\left(\boldsymbol{w}^{T} \boldsymbol{z}\right)^{2}\right\}=\|\boldsymbol{w}\|^{2}=1$ to keep the variance of $\boldsymbol{w}^{T} \boldsymbol{z}$ constant. The algorithm is presented in

$$
\begin{gathered}
\Delta \boldsymbol{w} \propto \gamma E\left\{\boldsymbol{z} g\left(\boldsymbol{w}^{T} \boldsymbol{z}\right)\right\} \\
\boldsymbol{w} \leftarrow \frac{\boldsymbol{w}}{\|w\|}
\end{gathered}
$$

where $\gamma=E\left\{G\left(\boldsymbol{w}^{T} \boldsymbol{z}\right)\right\}-E\{G(v)\}$. The function $\mathrm{g}$ is the derivative of $\mathrm{G}$ used earlier. This gradient algorithm described above is commonly called the "one-unit" algorithm and can be used for finding one maximally non-gaussian direction.

The next step in the ICA process is to estimate several independent components. There are two selectable methods used by the FastICA code. The first method is called deflationary orthogonalization. This method is presented in Figure 2-3. The second of the methods for estimation of multiple independent components is called the symmetric orthogonalization method and is presented in Figure 2-4. It can be seen that both methods contain an orthogonalization step after each step in estimation of unmixing matrices $\mathbf{w}$. This step is necessary due to the fact that the vectors $\mathbf{w}_{\mathrm{i}}$ corresponding to different independent components are orthogonal in the whitened space. As part of the preprocessing the data is whitened to reduce computational complexity. This is accomplished through whitening by reducing the degrees of freedom it is necessary for the algorithm to span during the search for the mixing matrix. It can be shown that in large dimensioned data sets the reduction in the computational load due to whitening is $~ 50 \%$. 


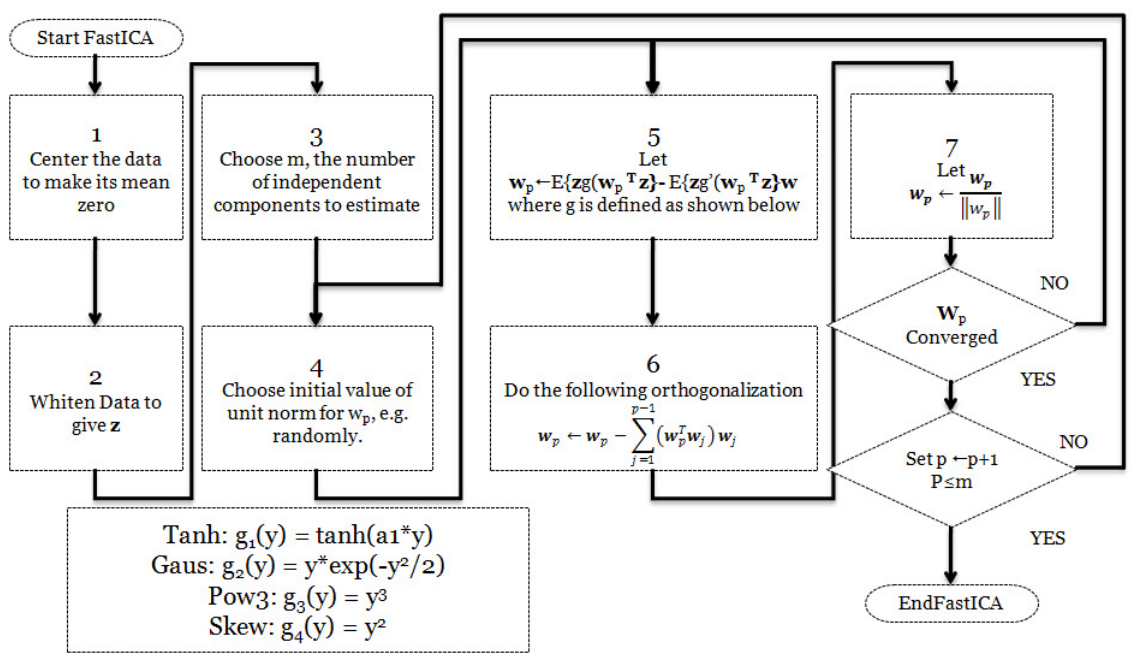

Figure 2-3 FastICA algorithm for estimating several ICs with deflationary orthogonalization

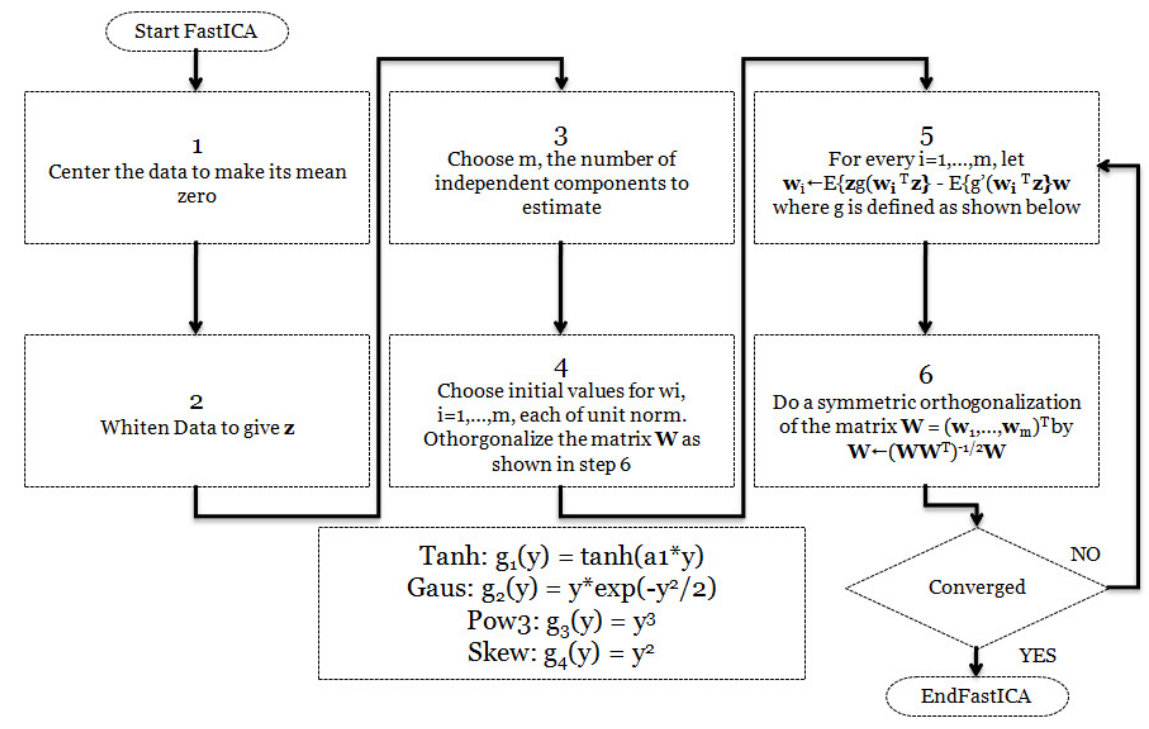

Figure 2-4 FastICA algorithm for estimating several ICs with symmetric orthogonalization

\subsection{ICA Application to Radar Scattering}

For this thesis, the FastICA algorithm is applied to radar return signals over a suite of observation angles. The premise of this undertaking is to observe whether the responses due to scatterers are related to the independent components extracted by ICA.

ISAR imaging techniques have been employed to enable a readily interpretable technique for visualization of the results of the application of ICA. The synthetic array data set 
naturally lends itself to analysis by ICA. The structure of the data being examined is a two dimensional array with rows and columns corresponding to observation angles and observation times. 


\section{CHAPTER 3}

\section{EXPERIMENTAL CONFIGURATION}

This study has been performed using scattering data taken in the anechoic chamber of the Wave Scattering Research Center (WSRC) at the University of Texas at Arlington. For this experiment the chamber was configured as a turntable ISAR system. The heart of the lab's measurement system is a Hewlett Packard (HP) 8510A network analyzer. The frequency range capability of this analyzer is $45 \mathrm{MHz}$ to $26.5 \mathrm{GHz}$. To enable freespace measurements using the analyzer an amplifier is need. The amplifier used in the WSRC configuration is HP 8349B. This device is the frequency limiting factor within the measurement set with an operational range of $2-$ $18 \mathrm{GHz}$. For transmit and receive antennas, the WSRC facility uses dual polarized conical horn antennas, model A6100, manufactured by the Dalmo Victor Division of the Singer Company. A schematic of the equipment configuration is shown in Figure 3-1. 


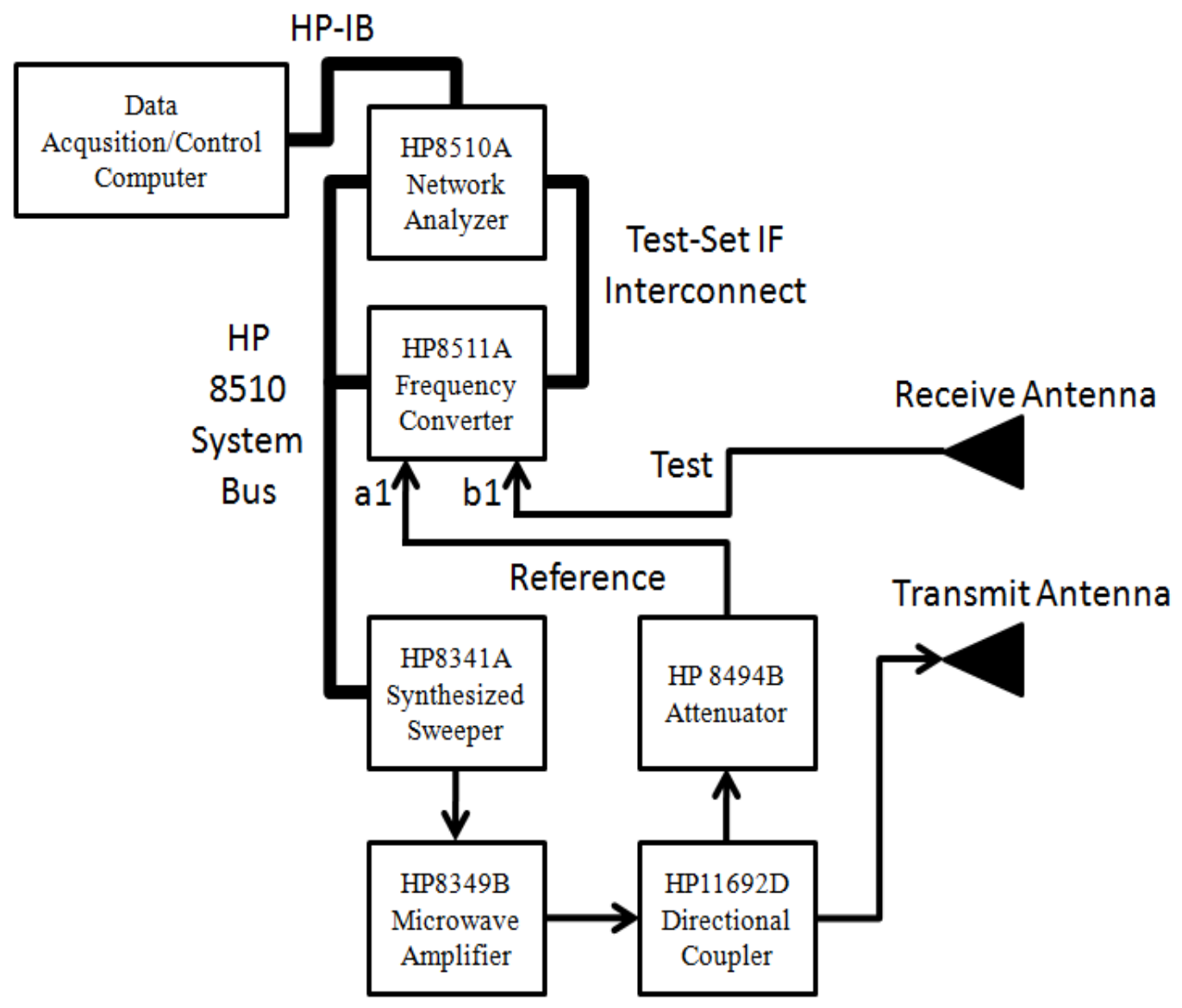

Figure 3-1 WSRC Measurement Equipment Schematic

The measurement parameters used for the experiments presented in this paper are shown in Error! Reference source not found.. For these experiments a single elevation angle was chosen and the motion of the target was constrained about the azimuthal axis of the turntable. A bandwidth of $4 \mathrm{GHz}$ was used along with the maximum number of frequency points that the equipment is capable of acquiring. 
Table 3-1 Experimental Measurement Parameters

\begin{tabular}{|c|c|c|}
\hline \multicolumn{4}{|c|}{ Frequencies } \\
\hline Start Frequency & 4 & $\mathrm{GHz}$ \\
\hline Stop Frequency & 8 & $\mathrm{GHz}$ \\
\hline Num. of Points & 801 & \\
\hline \multicolumn{3}{|c|}{ Elevation Angles } \\
\hline Start Angle & 45 & Degrees \\
\hline Stop Angle & 45 & Degrees \\
\hline Step Size & 0 & Degrees \\
\hline \multicolumn{3}{|c|}{ Azimuth Angles } \\
\hline Start Angle & 0 & Degrees \\
\hline Stop Angle & 360 & Degrees \\
\hline Step Size & 5 & Degrees \\
\hline
\end{tabular}

Before measurement of a target set it is necessary to perform a measurement calibration of the equipment. This calibration technique involves the placement of a target of known radar cross-section onto the turntable and performing a set of measurements over the frequency and angular ranges that are to be used for the target of interest. These measurements are then compared to the expected result for the known target. Any differences as a result of the comparison are used as bias error terms to compensate for imperfections in the measurement setup. For results presented in this paper a 4" sphere was used as the reference target and complex error terms were derived using the well know Mei Series expansion.

Following system calibration, the target being analyzed is placed onto a turntable which has the ability to rotate the target to any azimuthal angle. This rotation of the target is the key to providing the relative motion between the sensor system and the target that is required for synthetic aperture processing. Since it is the target motion about the radar system this configuration is of the Inverse Synthetic Aperture ilk.

The measurements taken in the WSRC chamber are automated using a computer algorithm to position the turntable, trigger the HP8510 system and record the measurement data. A flow diagram outlining the steps in the measurement process is shown in Figure 3-2. 

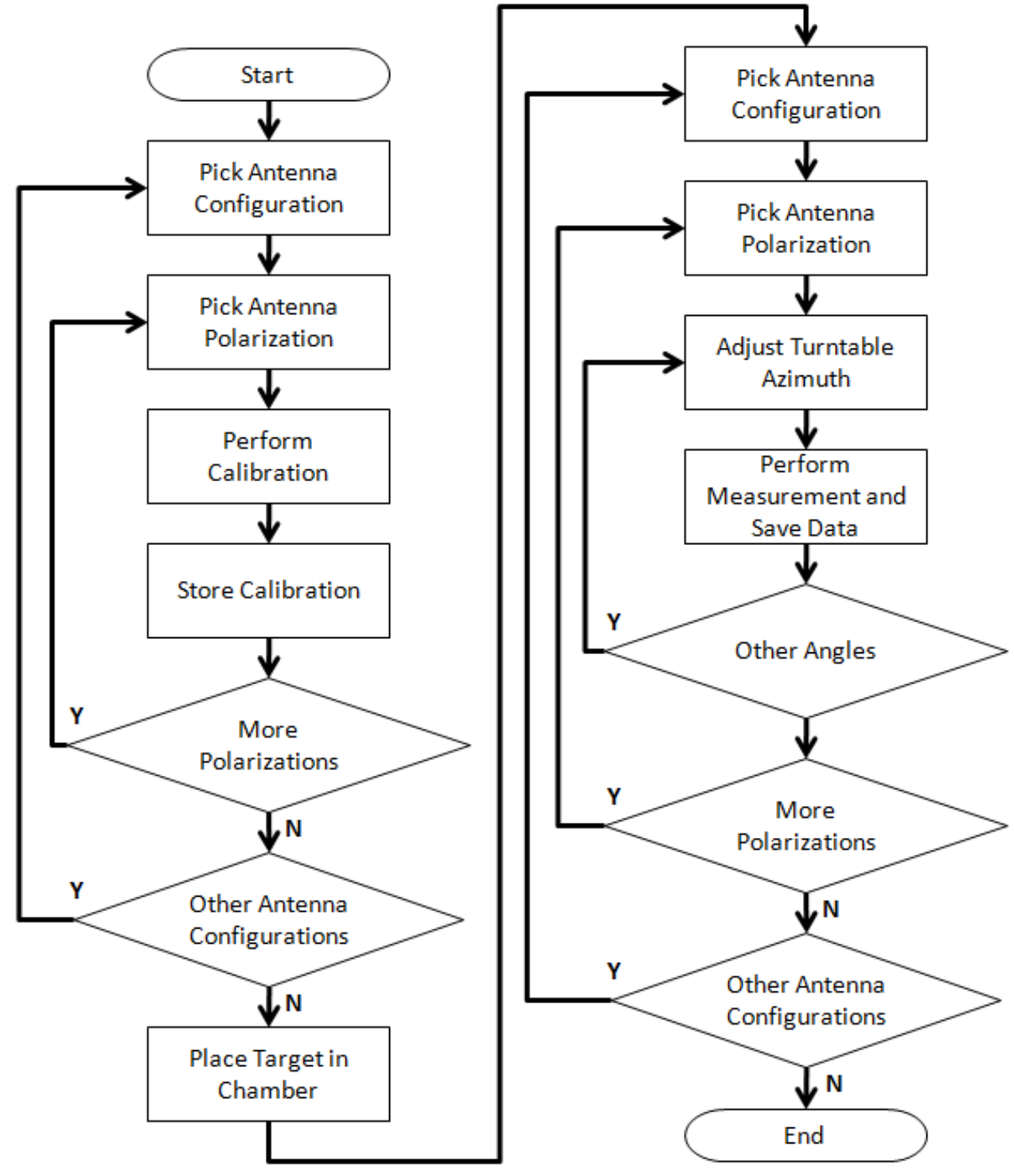

Figure 3-2 Measurement Routine Flow Chart 


\section{CHAPTER 4}

\section{EXPERIMENTAL RESULTS}

The results of this study are shown in the following section. It will be shown that the application of ICA to electromagnetic scattering data has promise in extracting additional information about the underlying components contained in the measurement data. For most targets, the measured radar reflection is a conglomerate due to a variety of scattering mechanisms. The purpose of this study is to assess the ability of a well known source separation technique, Independent Component Analysis (ICA), to decompose the conglomeration of sources present in a radar measurement. The separation of the independent scattering sources, through the application of ICA, has the potential to allow for additional information about a target to be extracted. This study is performed in a qualitative framework as this is the first known study of the application of the ICA to synthetic aperture data. It is expected that later studies will produce a more quantitative measure of the performance of the algorithm in this domain. Therefore the assessments made in this section will be, for the most part, qualitative.

Presented below are the results of the application of ICA to ISAR imaging using multiple targets. In all cases, the target set was interrogated using microwaves in 4-8 GHz frequency range with 801 points. The polarization was vertical in both transmission and reception. These measurements were made on the turntable system at in the anechoic chamber of the Wave Scattering Research Center at the University of Texas at Arlington. A single antenna elevation angle was used and the azimuthal angle was stepped between 0 and 360 degrees in 5 degree increments. 
Frequency domain data was captured over the angular domain and then calibrated in range and phase. Following calibration, the frequency domain data was converted to the time domain [18]. To limit the noise processed by the algorithm, most of the non-target data within the scene was then removed using a rectangular time window of $6.75 \mathrm{~ns}$. The window was centered at $t=0$. This window served to remove any return data which was not within $\pm 3.375 \mathrm{~ns}$ of the calibration reference point. The resulting windowed time domain data was then operated on by the FastICA algorithm. The application of the FastICA algorithm produced a set of unmixing matrices which were applied to the original windowed time domain data. The consequence of the application of the unmixing matrices is the production of sets of independent components. The time-domain independent components are converted back to the frequency domain for ISAR analysis and comparison to the original data.

The FastICA algorithm has selectable options for the orthogonalization approach, nonlinear function (higher-order statistics estimator), number of eigenvalues to be used in the whitening process, and the number of independent components extracted. The range of available choices for these parameters is depicted in Table 4-1.

Table 4-1 FastICA Parameter Selections

\begin{tabular}{|r|l|}
\hline \multicolumn{1}{|c|}{ Selectable Parameter } & \multicolumn{1}{|c|}{ Values } \\
\hline Orthogonalization Approach & Deflation \\
\hline & Symmetric \\
\hline Nonlinear Function & Tanh \\
\hline & pow3 \\
\hline & Gaus \\
\hline & Skew \\
\hline Number of Independent Components & 1 up to Dimension of Data \\
Estimated & \begin{tabular}{l} 
Data \\
\hline
\end{tabular} \\
\hline
\end{tabular}

To establish the effect of the above parameters on the performance of the FastICA algorithm on the ISAR data, a simple offset 6" sphere target will be used. Following the establishment of a parameter selection rational, a wide set of targets will be presented. These 
targets include a 4" offset sphere, a combination of the 6" and 4" spheres and a target made up of a 4" sphere and set of three metallic cylinders arranges in a triangle. Each target set will be presented with a photo to aid in the readers understanding of the configuration of the scattering elements in the target. An image of the first target is presented in Figure 4-1. The target is a 6" diameter sphere offset from the center of the turntable by $4 "$.

Time gating is used to limit the impact of non-target data which can be viewed as noise when considered in the estimating framework of ICA. The effect of this noise is to limit the accuracy with which the underlying components can be estimated. The time gate placed around the target data corresponds to $\pm 1 \mathrm{~m}$ in distance. Of concern with application of the time gate would be the creation of sidelobes in the frequency domain and the potential to gate out data related to the desired target response. The effect of significant sidelobes due to truncation of the time domain data would be seen as periodic circular ripples in the spatial domain of the SAR image. It is known that the scattering response of a sphere has delayed resonant components related to the Mie scattering. The time gate applied in this study allows for transversal of the circumference of a 6 " sphere 4 times. To ensure that the effect of time gating has not impacted the fidelity of the scattering data, an ISAR image prior to time gating is shown in Figure 4-2(a) for comparison to the image created from the time gated data shown in Figure 4-2(b). The difference of the two images is presented in Figure 4-3. 

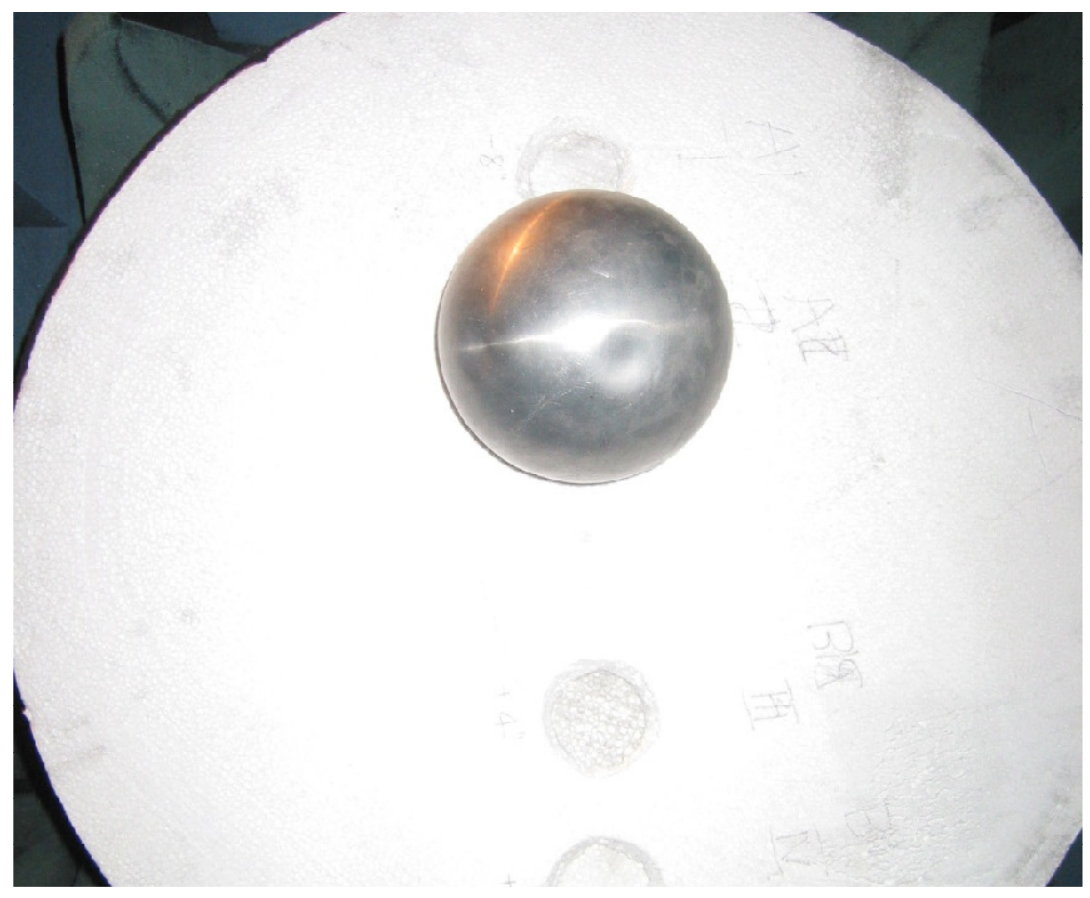

Figure 4-1 Photo of offset 6" sphere 


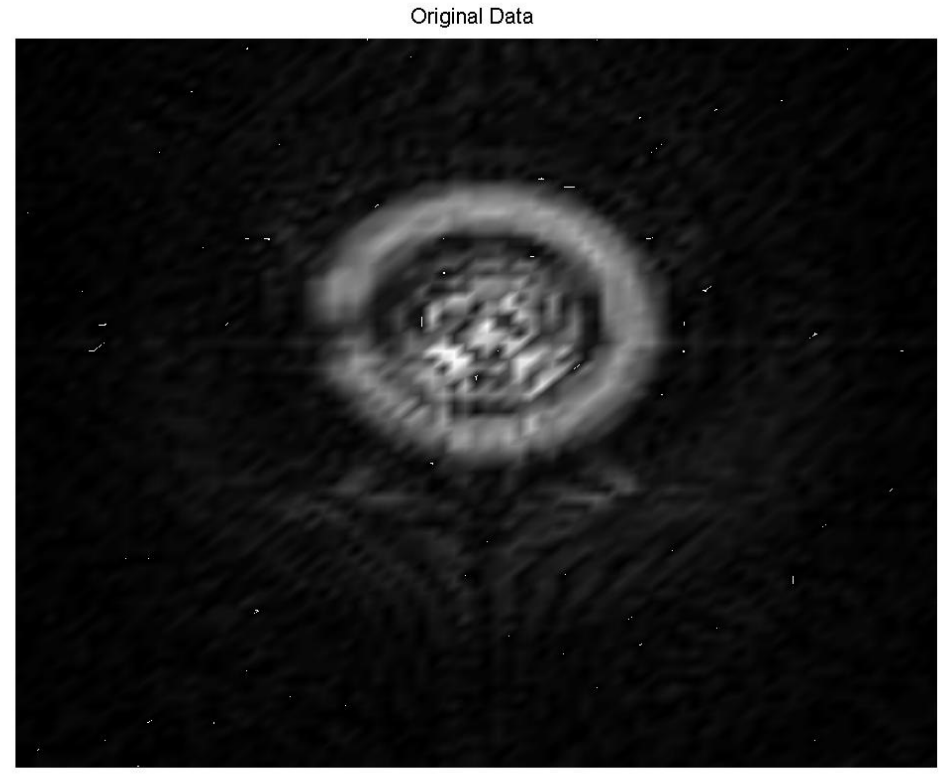

(a)

Time Windowed Data : Window Length $=6.75 \mathrm{e}-009 \mathrm{~s}$

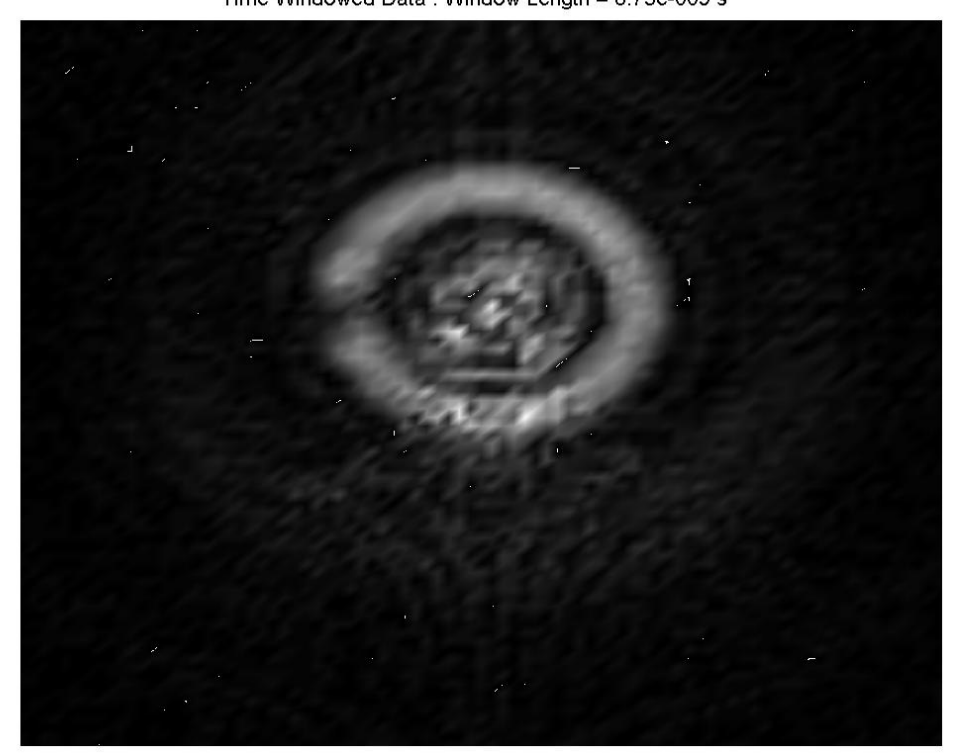

(b)

Figure 4-2 (a) ISAR image formed with raw scattering data (b) ISAR image formed from timegated scattering data 


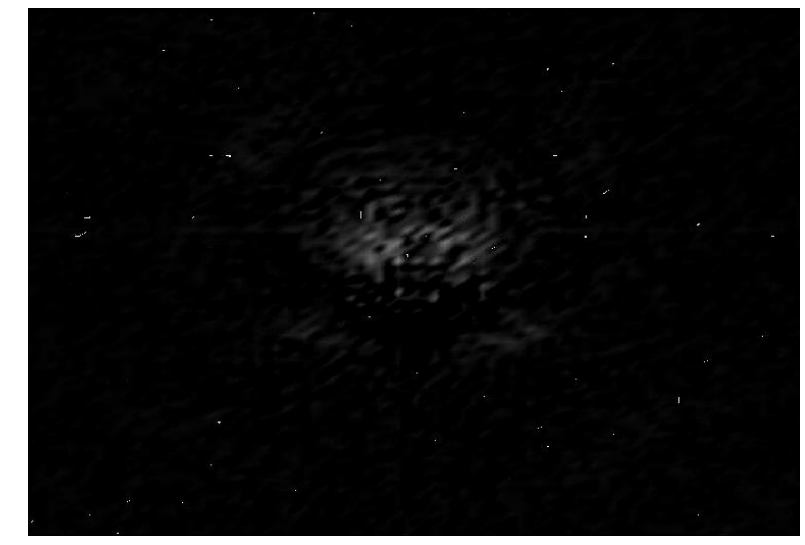

Figure 4-3 Difference between non-time gated image and time gated image

\subsection{Orthogonalization Approach Parameter Selection}

The first parameter selection addressed was the orthogonalization approach. The data presented in Figure 4-4 through Figure 4-21 enables a qualitative comparison of the two approaches. The results of ICA using each of the two approaches are presented. In these cases, the other selectable parameters were held static. For examination of the effect of the approach parameter the nonlinear function was chosen to be the Gaussian function, the Eigenvalues used in the whitening process was chosen to be nine and the independent components to be extracted was varied from one to nine. 
ICA Processed Data: Component \# 1 of 1 / Total Eigenvalues: 9

Window Length $=6.75 \mathrm{e}-009 \mathrm{~s} /$ Method: gaus

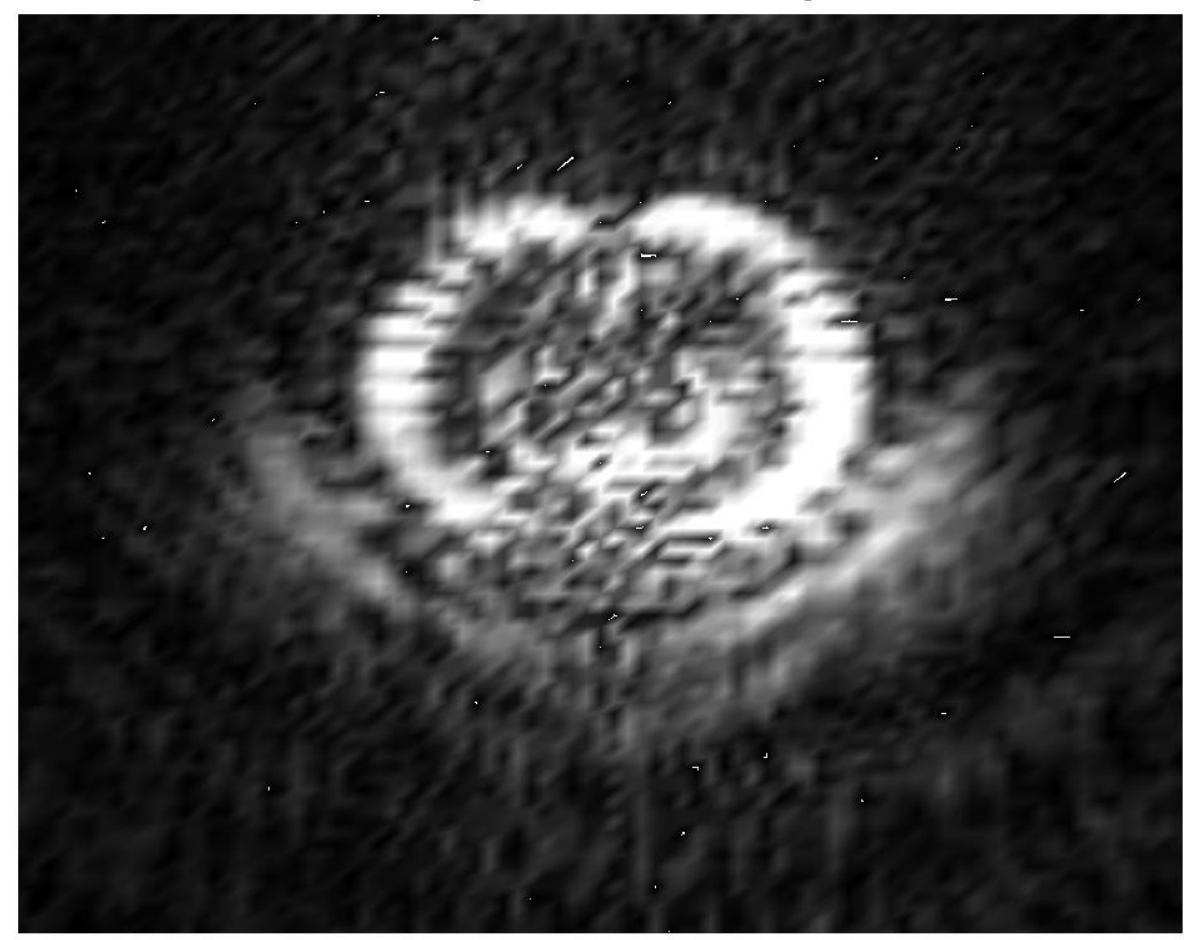

Figure 4-4 ICA results with 1 component using deflation orthogonalization 
ICA Processed Data: Component \# 1 of 1 / Total Eigenvalues: 9 Window Length $=6.75 \mathrm{e}-009 \mathrm{~s} /$ Method: gaus

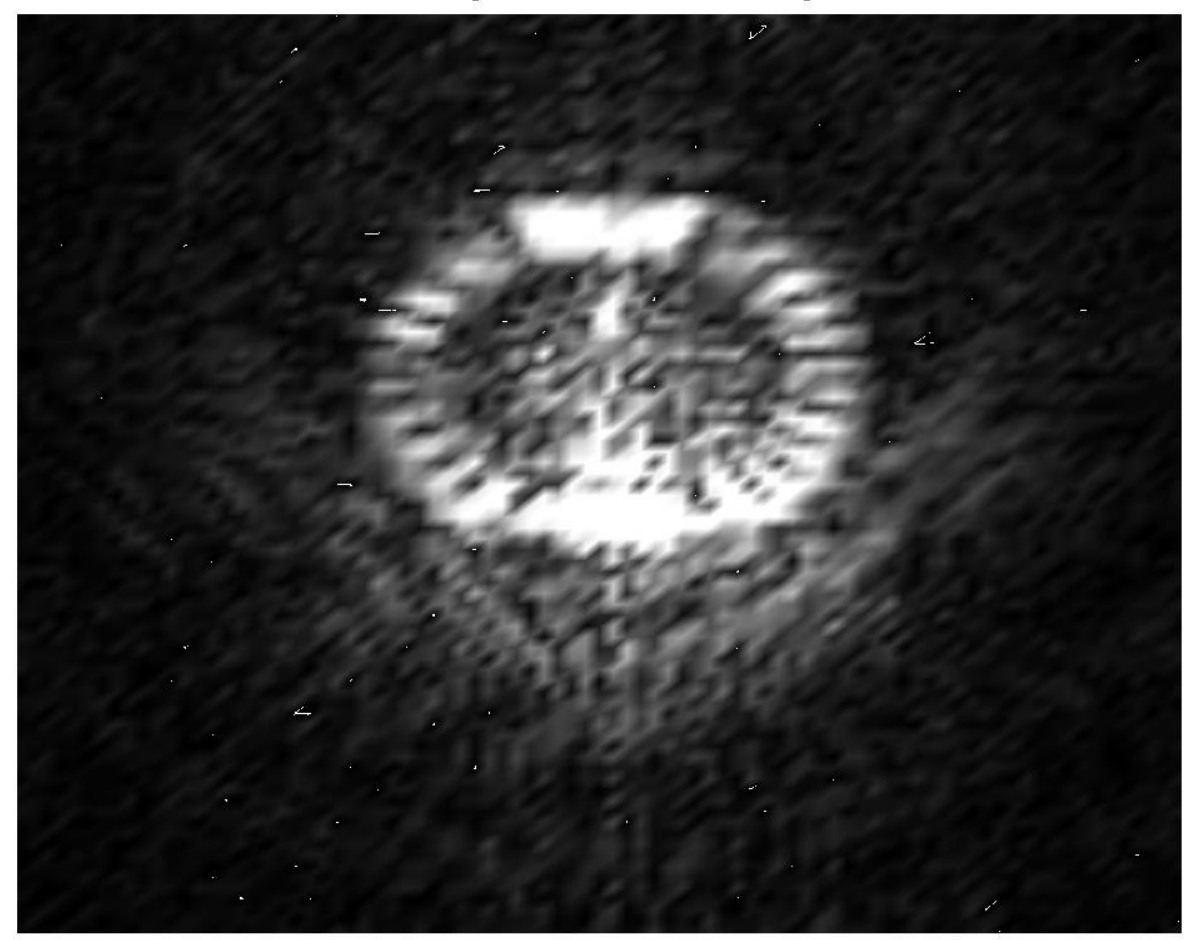

Figure 4-5 ICA results with 1 component using symmetric orthogonalization 
ICA Processed Data: Component \# 1 of 2 / Total Eigenvalues: 9 Window Length $=6.75 \mathrm{e}-009 \mathrm{~s} /$ Method: gaus

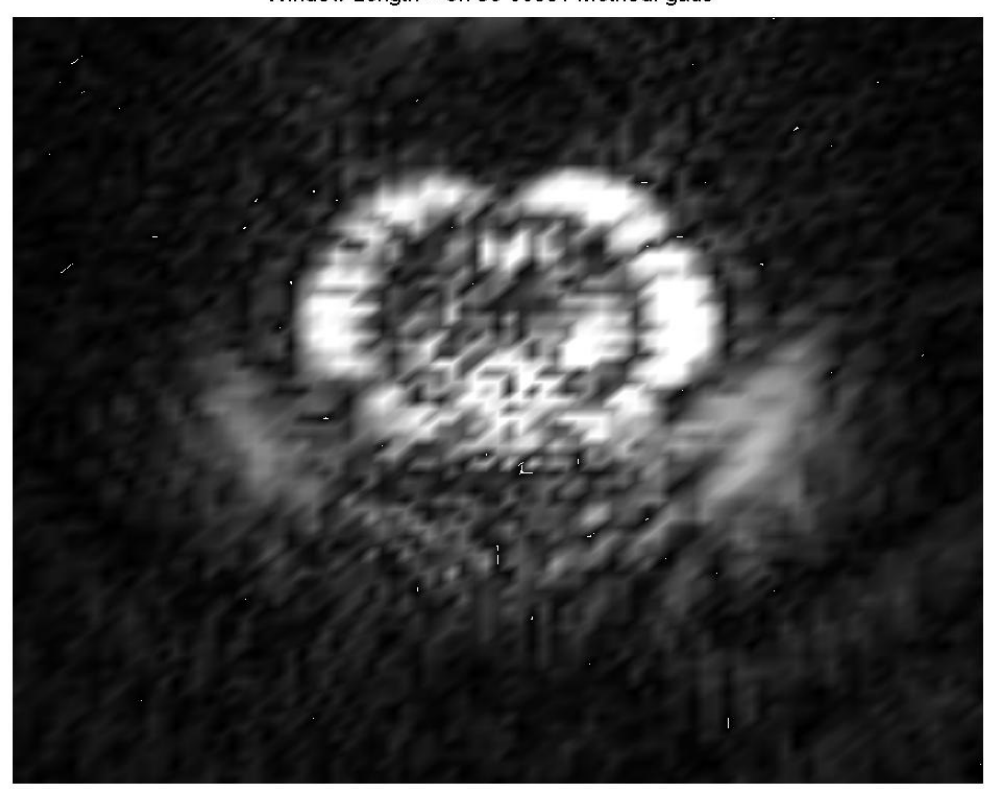

(a)

ICA Processed Data: Component \# 2 of 2 / Total Eigenvalues: 9 Window Length $=6.75 \mathrm{e}-009 \mathrm{~s} /$ Method: gaus

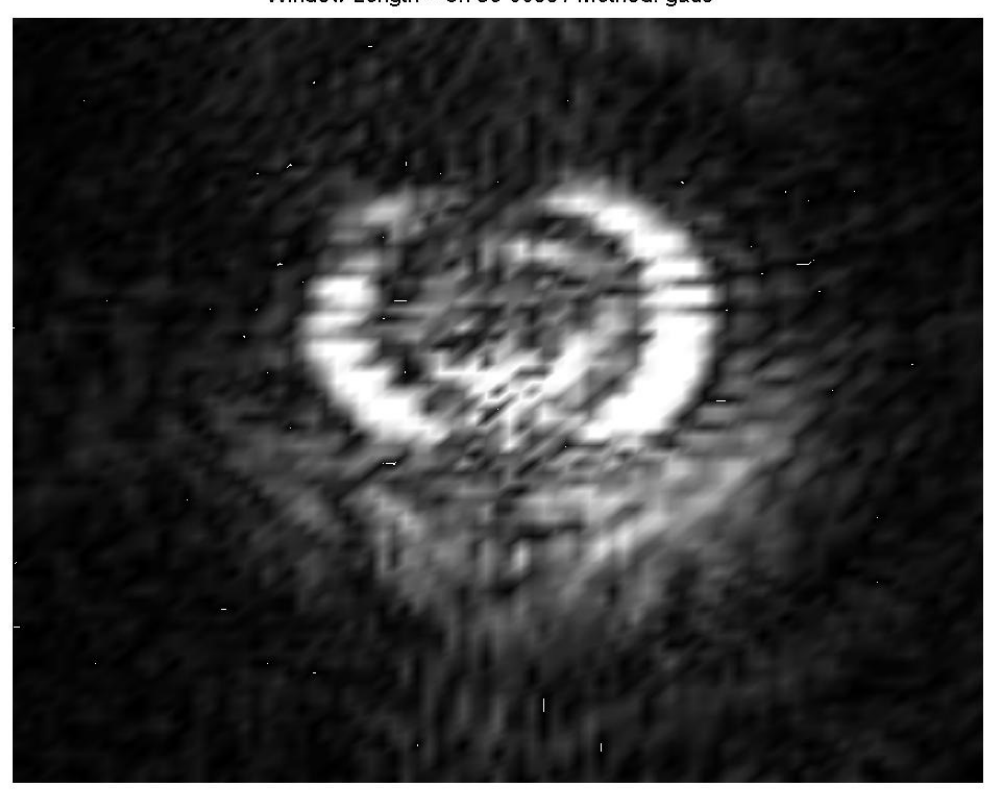

(b)

Figure 4-6 ICA results using deflation orthogonalization (a) component \#1 (b) component \#2 
ICA Processed Data: Component \# 1 of 2 / Total Eigenvalues: 9

Window Length $=6.75 \mathrm{e}-009 \mathrm{~s} /$ Method: gaus

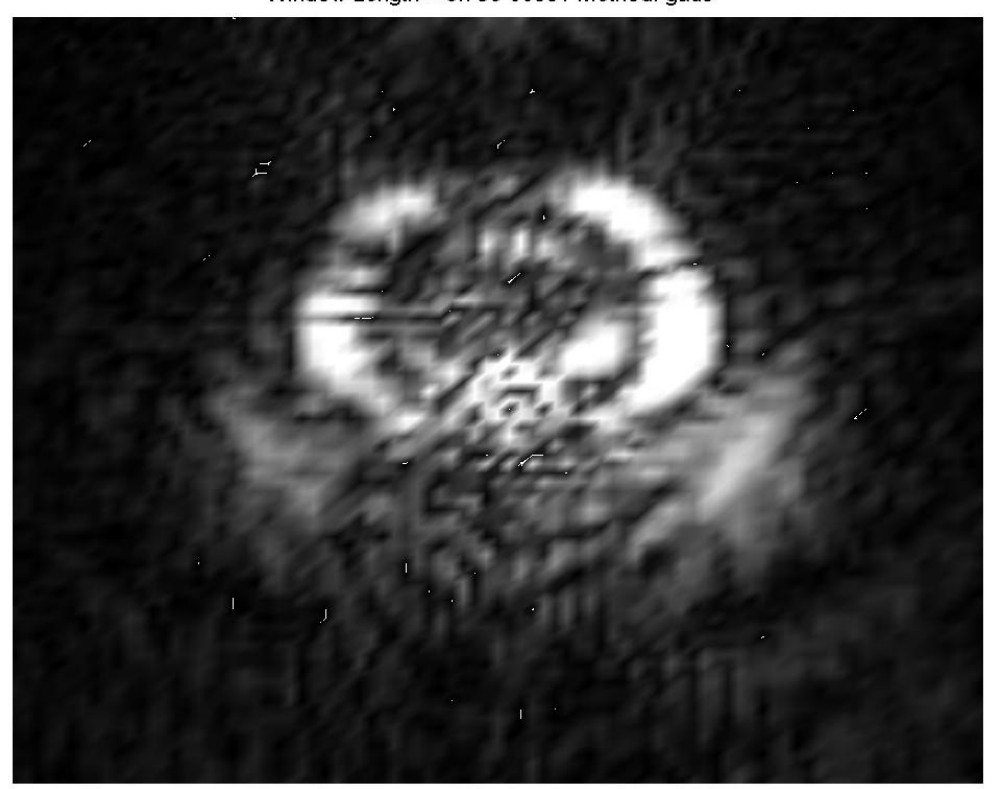

(a)

ICA Processed Data: Component \# 2 of 2 / Total Eigenvalues: 9 Window Length $=6.75 \mathrm{e}-009 \mathrm{~s} /$ Method: gaus

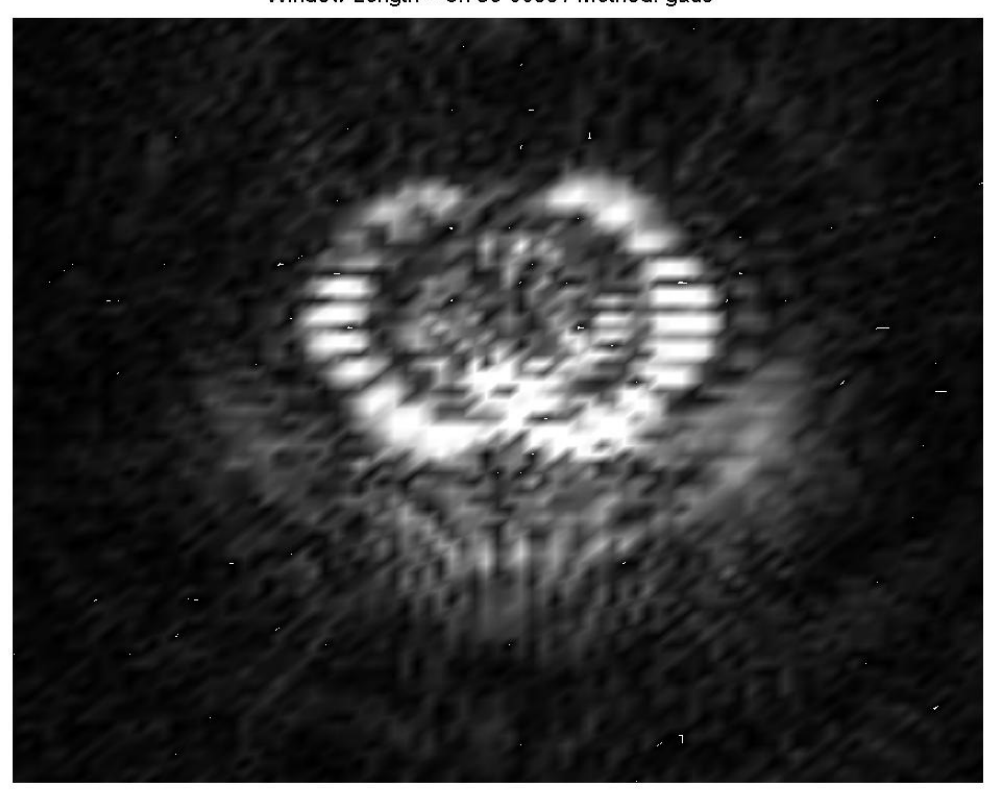

(b)

Figure 4-7 ICA results using symmetric orthogonalization (a) component \#1 (b) component \#2 


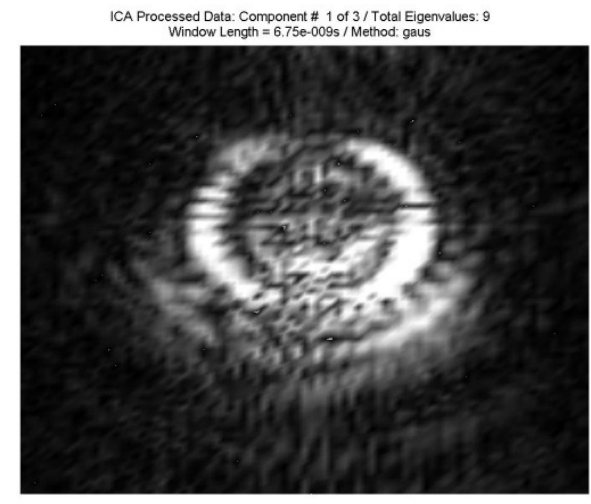

(a)
ICA Processed Data: Component \# 2 of $3 /$ Total Eigenvalues: 9
Window Length $=6.75-009$ s $/$ Method gaus

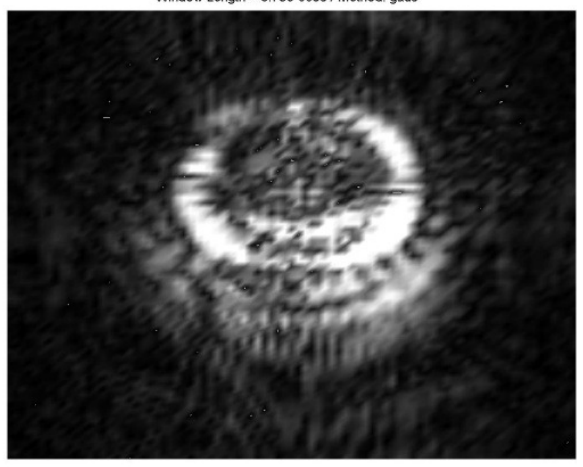

(b)

ICA Processed Data: Component \# 3 of $3 /$ Total Eigenvalues: 9
Window Length $=6.75$ - 009 s $/$ Method: gaus

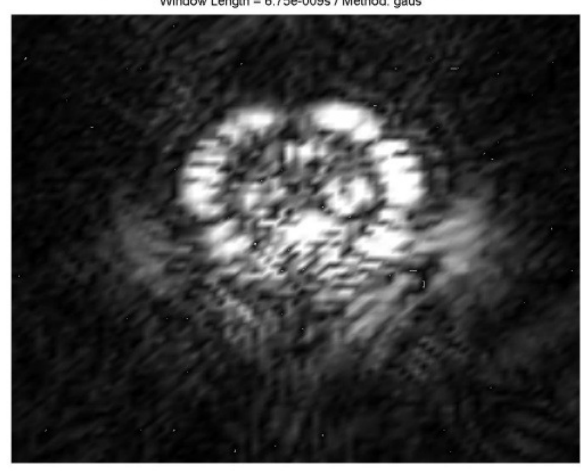

(c)

Figure 4-8 ICA results using deflation orthogonalization (a) component \#1 (b) component \#2 (c) component \#3 


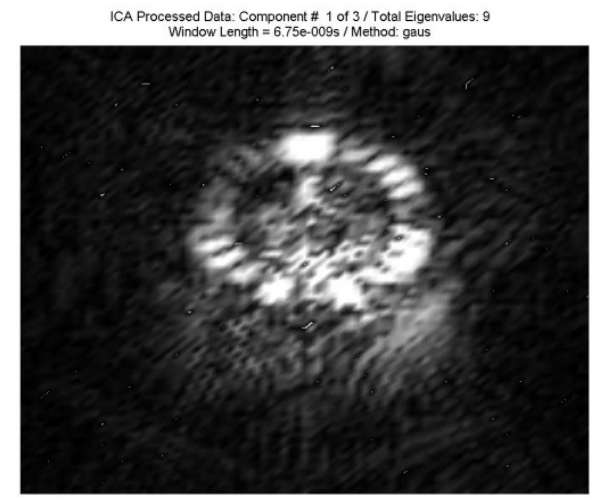

(a)

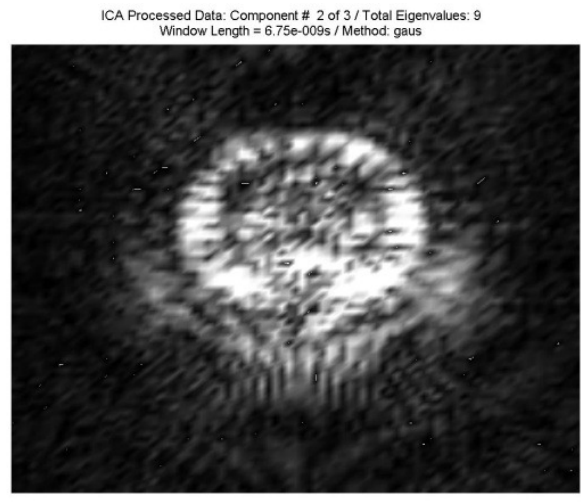

(b)

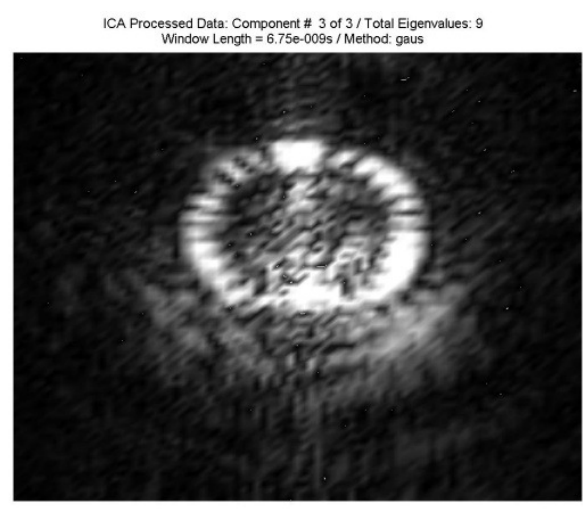

(c)

Figure 4-9 ICA results using symmetric orthogonalization (a) component \#1 (b) component \#2 (c) component \#3 
ICA Processed Data: Component \# 1 of $4 /$ Total Eigenvalues: 9
Window Length $=6.75 \mathrm{e}-0095 /$ Method gaus

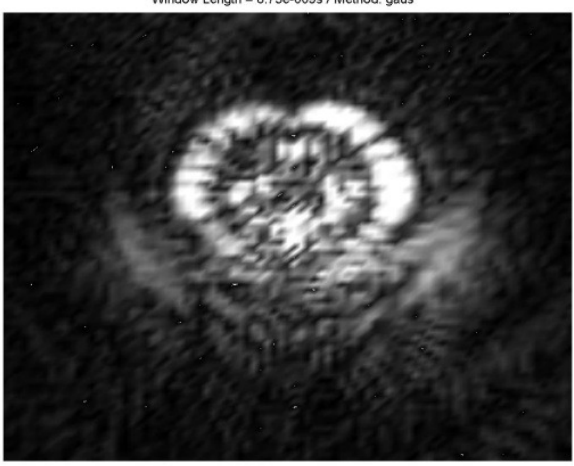

(a)

ICA Processed Data: Component \# 3 of 4 / Total Eigenvalues: 9
Window Lengoth $=6.75 \mathrm{e}-0095$ / Method: gaus

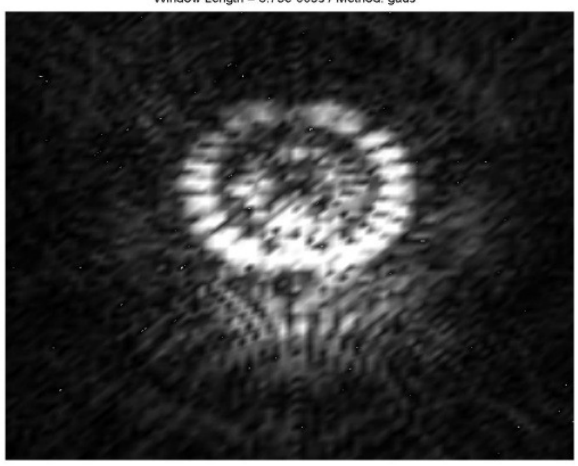

(c)
ICA Processed Data: Component \#2 2 of $4 /$ Total Eigenvalues: 9
Window Length $=6.756$ - 009 s $/$ Method: gaus

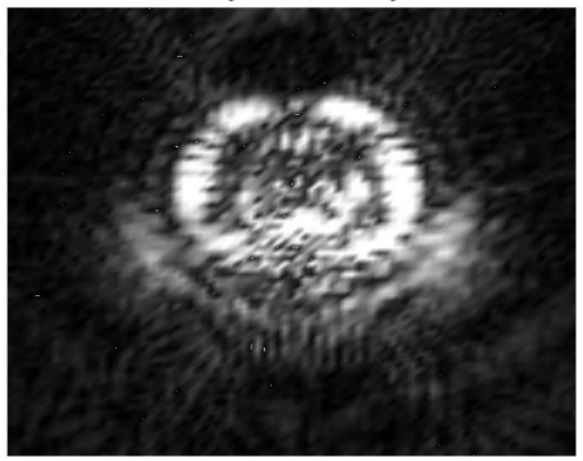

(b)

ICA Processed Data: Component \# 4 of $4 /$ Total Eigenvalues: 9
Window Length $=675$ - 009 s $/$ Method gaus

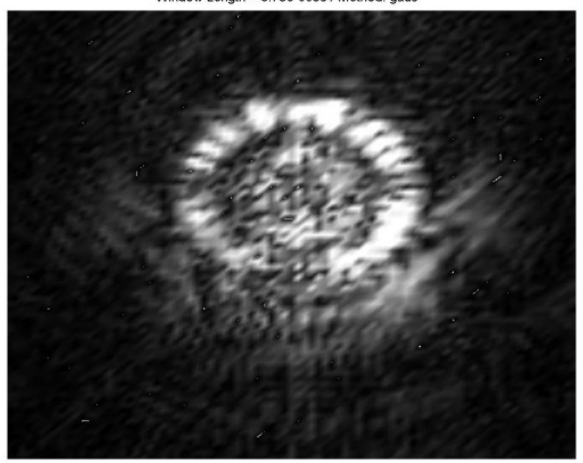

(d)

Figure 4-10 ICA results using deflation orthogonalization (a) component \#1 (b) component \#2 (c) component \#3 (d) component \#4 
ICA Processed Data: Component \# 1 of $4 /$ Total Eigenvalues: 9
Window Length $=6.75 e-0095$ / Method: gaus

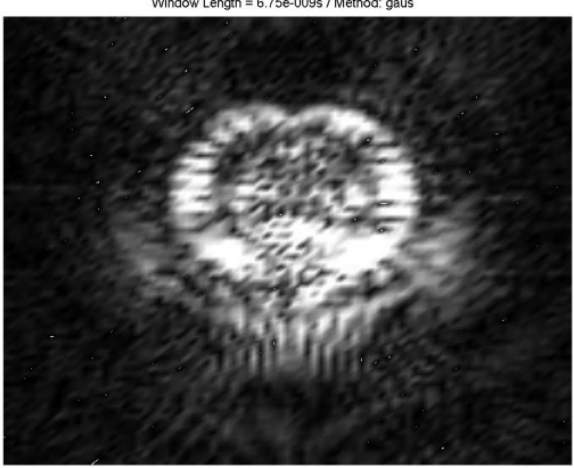

(a)

ICA Processed Data: Component \# 3 of 4 / Total Eigenvalues: 9
Window Lengoth $=6.75 \mathrm{e}-0095$ / Method: gaus

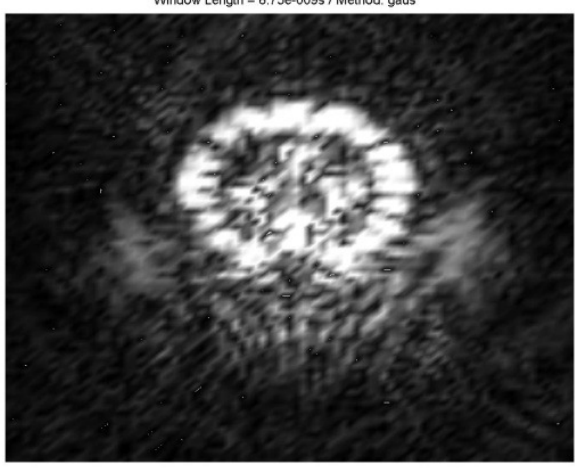

(c)
ICA Processed Data: Component \# 2 of $4 /$ Total Eigenvalues: 9
Window Length $=6.750-009$ s $/$ Method: gaus

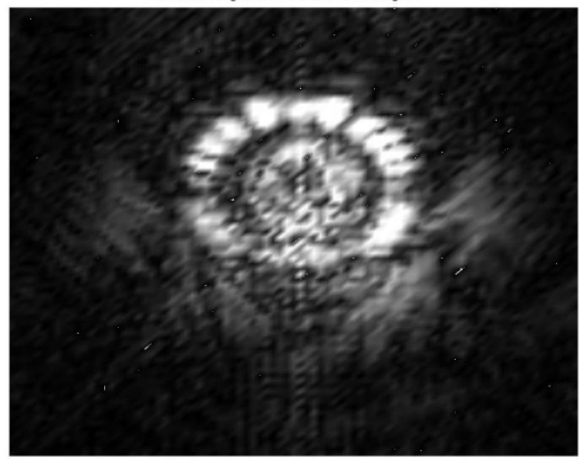

(b)

ICA Processed Data: Component \# 4 of $4 /$ Total Eigenvalues: 9
Window Length $=675$ - 009 s $/$ Method gavs

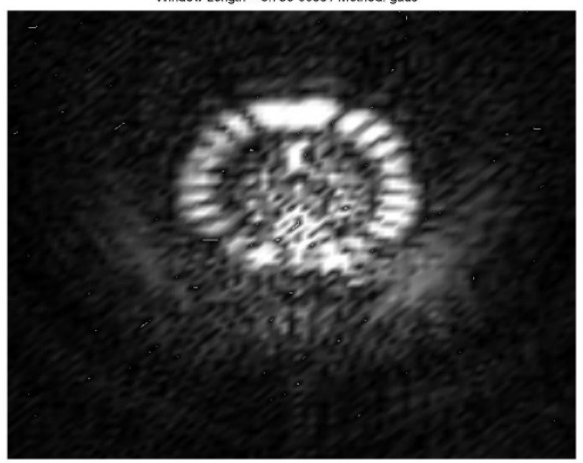

(d)

Figure 4-11 ICA results using symmetric orthogonalization (a) component \#1 (b) component \#2 (c) component \#3 (d) component \#4 
ICA Processed Data: Component \# 1 of $5 /$ Total Eigenvalues: 9
Window Length $=6.75 \mathrm{e}-0.09 \mathrm{~s} /$ Method; gaus

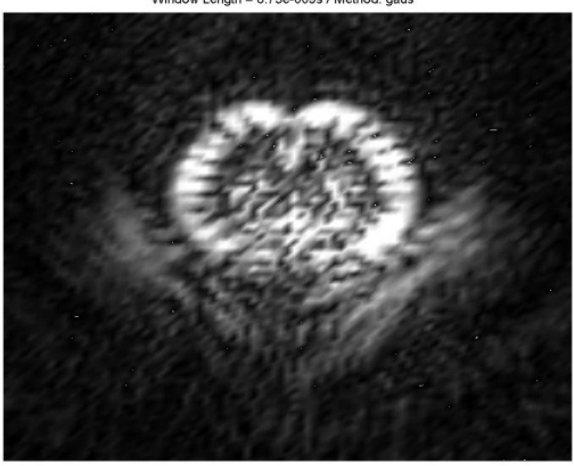

(a)

ICA Processed Data: Component \# 3 of 5 / Total Eigenvalues: 9
Window Lengoth $=675$. 75 -0095/ Method gaus

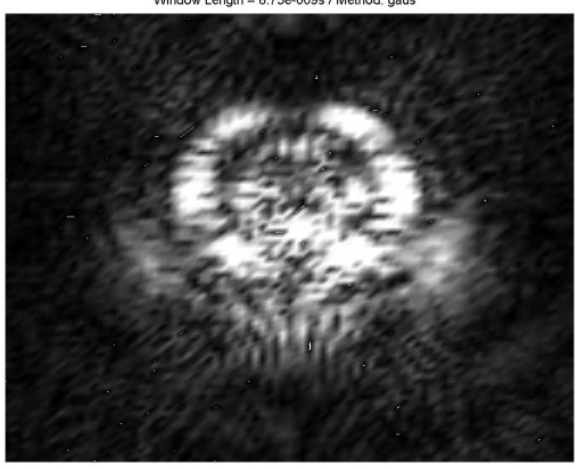

(c)
ICA Processed Data: Component \# 2 of $5 /$ Total Eigenvalues:
Window Length $=6.75$ - 009 s $/$ Method: gaus

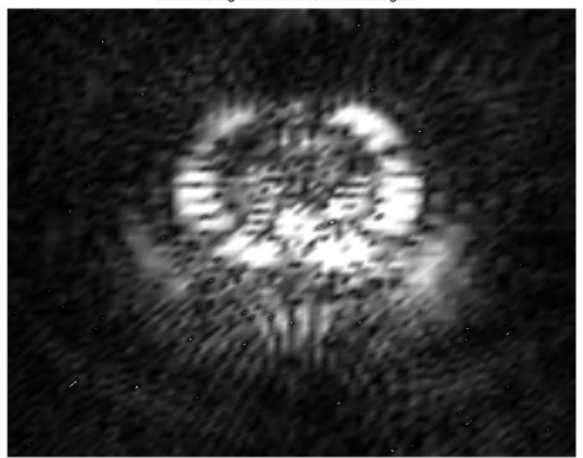

(b)

ICA Processed Data: Component \# 4 of $5 /$ Total Eigenvalues: 9
Window Length $=675$ - 009 s $/$ Method gaus

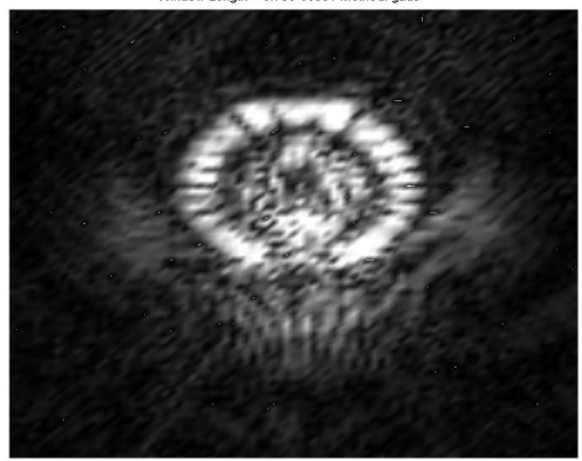

(d)

ICA Processed Data: Component \# 5 of $5 /$ Total Eigenvalues: 9

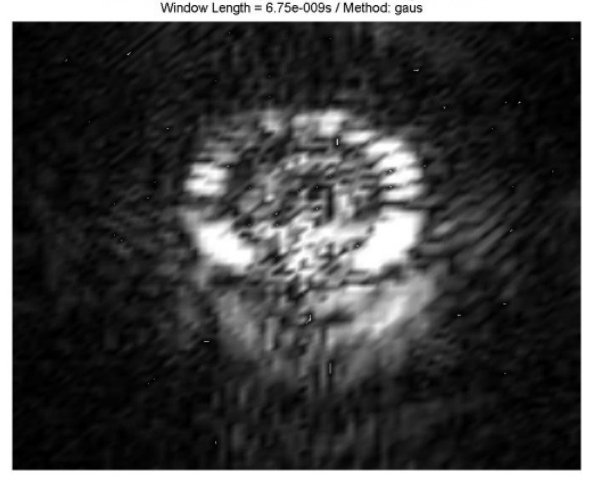

(e)

Figure 4-12 ICA results using deflation orthogonalization (a) component \#1 (b) component \#2 (c) component \#3 (d) component \#4 (e) component \#5 
ICA Processed Data: Component \# 1 of $5 /$ Total Eigenvalues: 9
Window Length $=6.75 \mathrm{e}-009 \mathrm{~s} /$ Method: gaus

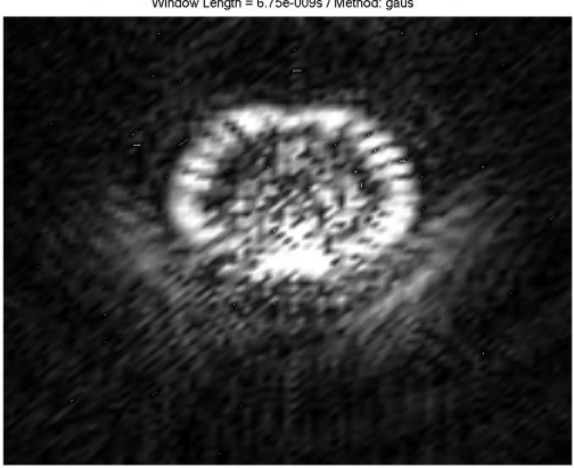

(a)

ICA Processed Data: Component\# 3 of $5 /$ Total Eigenvalues: 9
Window Length $=6.75$ - 009 s / Method: gaus

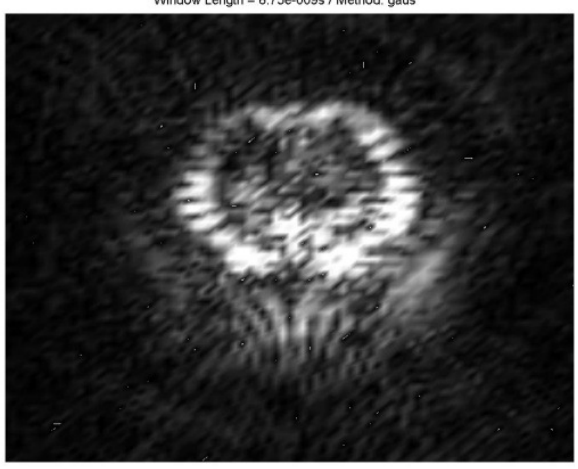

(c)
ICA Processed Data: Component \# 2 of $5 /$ Total Eigenvalues:
Window Length $=6.75$ - 009 s $/$ Method: gaus

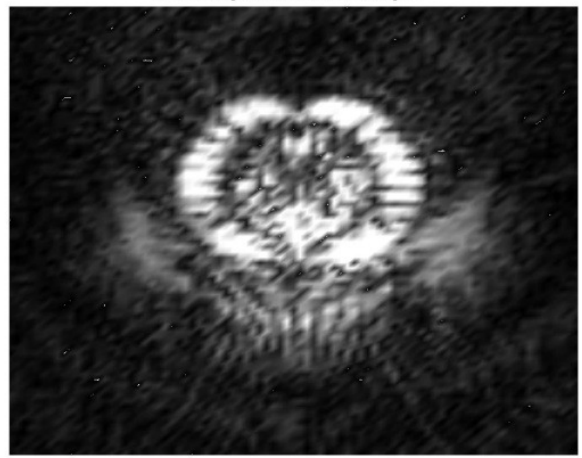

(b)

ICA Processed Data: Component \# 4 of $5 /$ Total Eigenvalues: 9
Window Length $=675$ - 009 s $/$ Method gaus

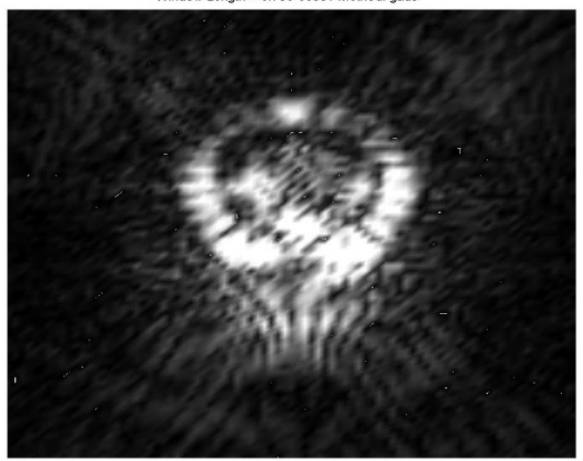

(d)

ICA Processed Data: Component \# 5 of $5 /$ Total Eigenvalues: 9

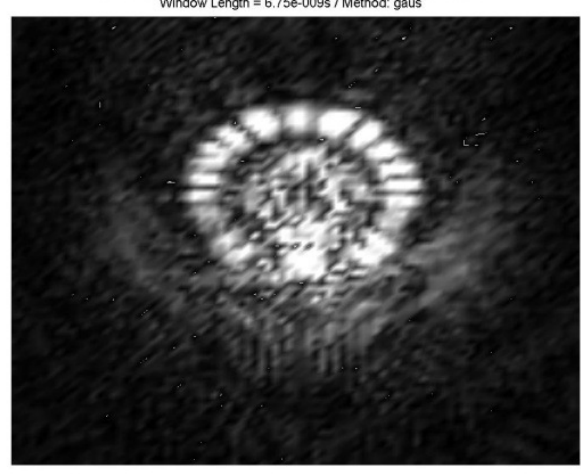

(e)

Figure 4-13 ICA results using symmetric orthogonalization (a) component \#1 (b) component \#2

(c) component \#3 (d) component \#4 (e) component \#5 
ICA Processed Data: Component \# 1 of $6 /$ Total Eigenvalues: 9
Window Length $=6.75 \mathrm{e}-009 \mathrm{~s} /$ Method: gaus

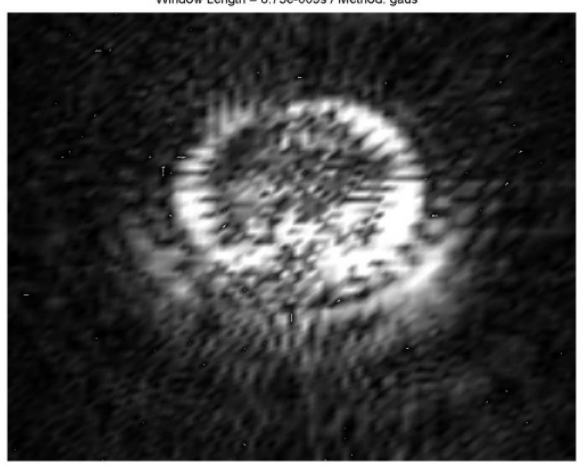

(a)

ICA Processed Data: Component\# 3 of $6 /$ Total Eigenvalues: 9
Window Length $=6.75$ - 009 s / Method: gaus

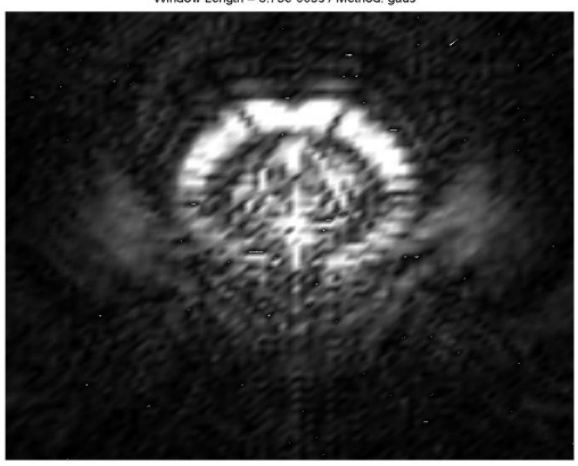

(c)

ICA Processed Data: Component \# 5 of $6 /$ Total Eigenvalues: 9

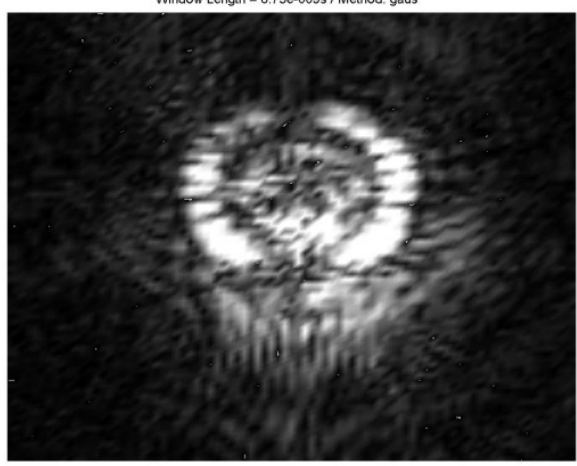

(e)
ICA Processed Data: Component \#2 2 of $6 /$ Total Eigenvalues: 9
Window Length $=6.756$ - 009 s $/$ Method: gaus

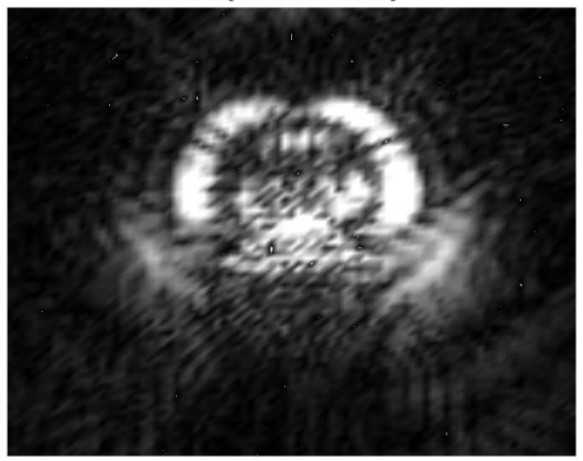

(b)

ICA Processed Data: Component \# 4 of $6 /$ Total Eigenvalues: 9
Window Length $=6.75$ - 009 s $/$ Method gaus

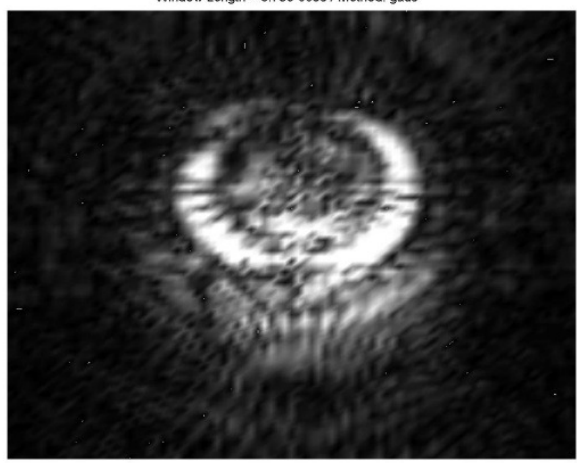

(d)

ICA Processed Data: Component \# 6 of $6 /$ Total Eigenvalues: 9
Window Length $=6.75$ - 009 s $/$ Method gous

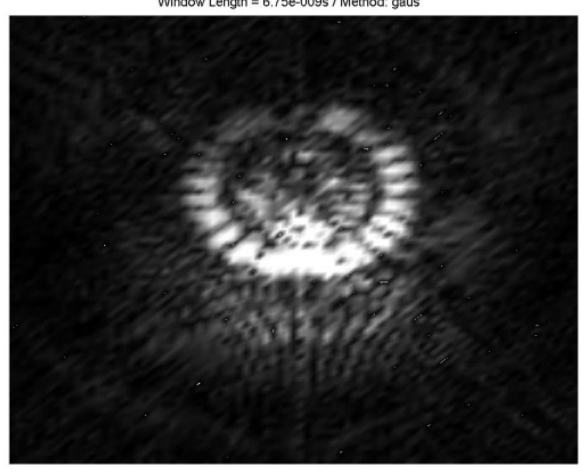

(f)

Figure 4-14 ICA results using deflation orthogonalization (a) component \#1 (b) component \#2 (c) component \#3 (d) component \#4 (e) component \#5 (f) component \#6 
ICA Processed Data: Component \# 1 of $6 /$ Total Eigenvalues: 9
Window Length $=6.75 \mathrm{t}-009 \mathrm{~s} /$ Method; gaus

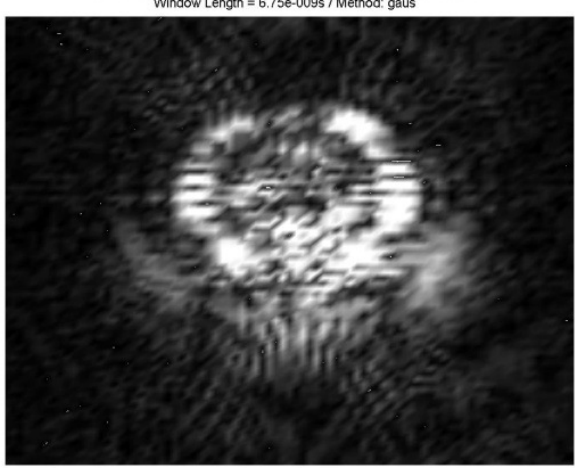

(a)

ICA Processed Data: Component \# 3 of 6 / Total Eigenvalues: 9
Window Lengoth $=6.75$ - 0095 / Method: gaus

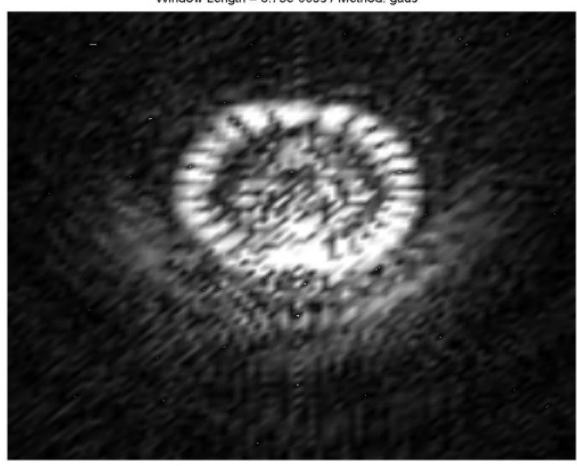

(c)

ICA Processed Data: Component \# 5 of $6 /$ Total Eigenvalues: 9

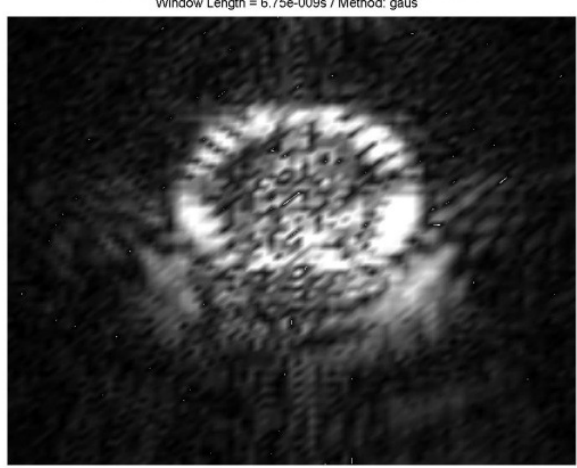

(e)
ICA Processed Data: Component \#2 2 of $6 /$ Total Eigenvalues:
Window Length $=6.756-009$ s $/$ Method: gaus

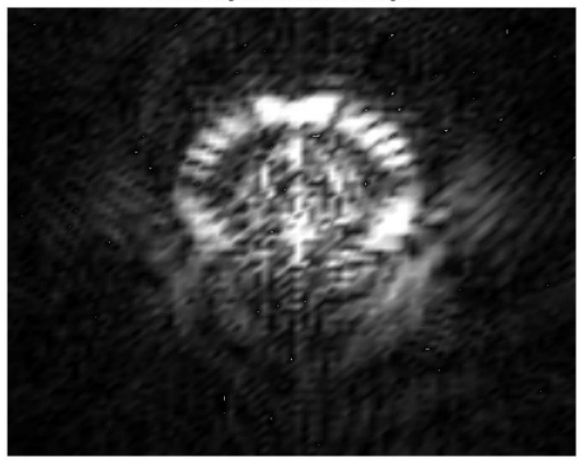

(b)

ICA Processed Data: Component \# 4 of $6 /$ Total Eigenvalues: 9
Window Length $=6.75 e-009$ s $/$ Method gaus

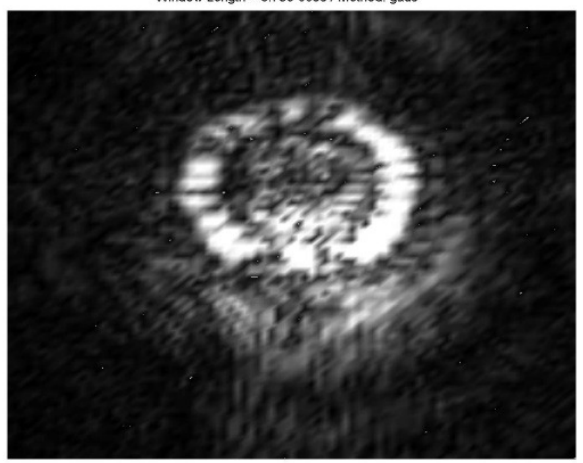

(d)

ICA Processed Data: Component \# 6 of $6 /$ Total Eigenvalues: 9
Window Length $=6.75$ - 009 s $/$ Method gous

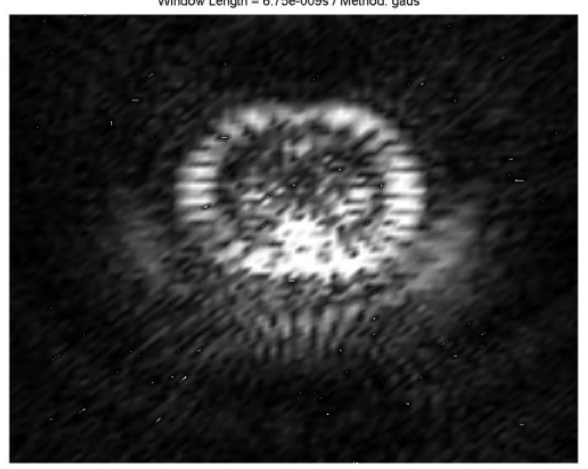

(f)

Figure 4-15 ICA results using symmetric orthogonalization (a) component \#1 (b) component \#2 (c) component \#3 (d) component \#4 (e) component \#5 (f) component \#6 


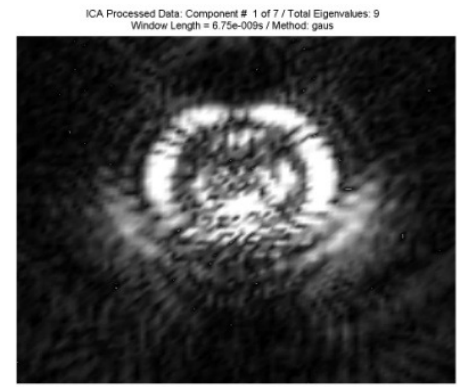

(a)

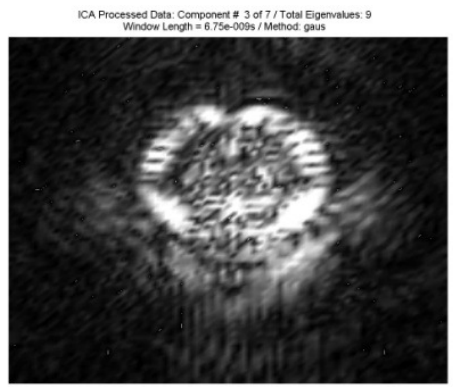

(c)

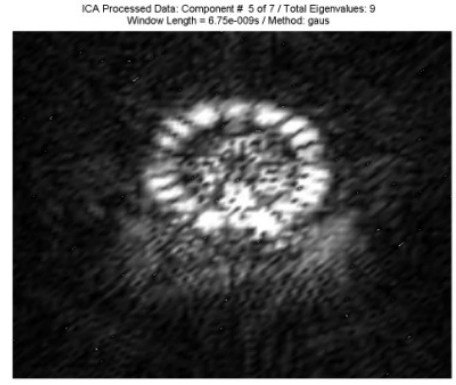

(e)

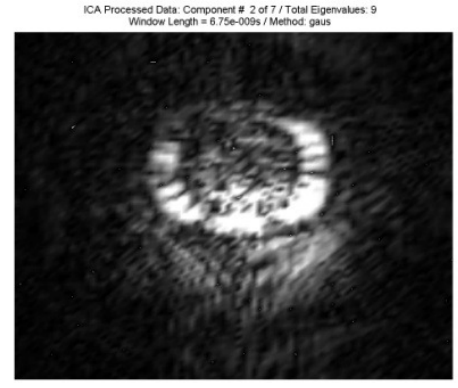

(b)

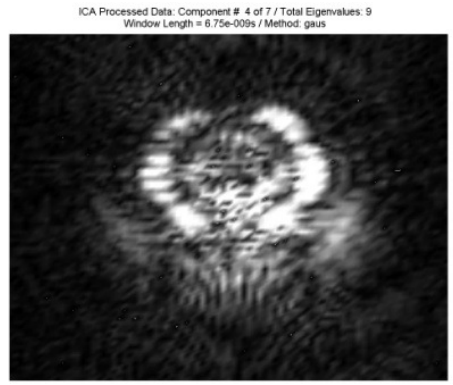

(d)

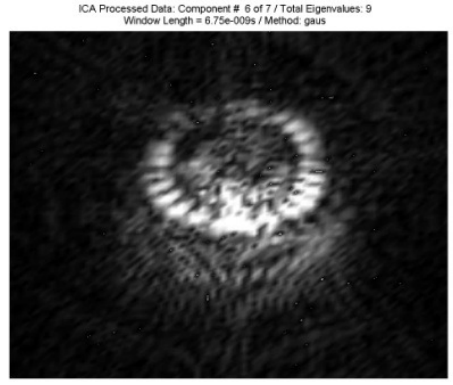

(f)

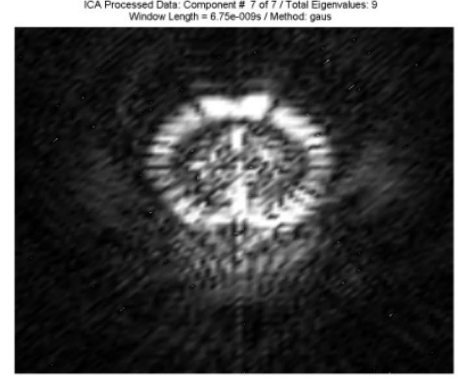

(g)

Figure 4-16 ICA results using deflation orthogonalization (a) component \#1 (b) component \#2 (c) component \#3 (d) component \#4 (e) component \#5 (f) component \#6 (g) component \#7 


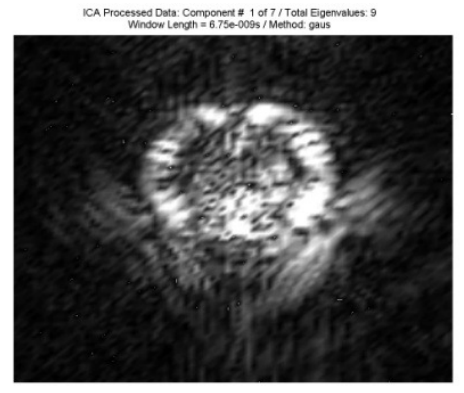

(a)

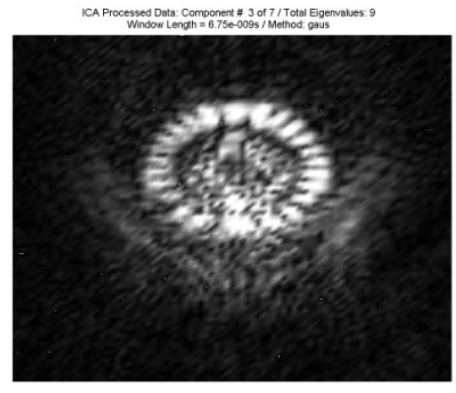

(c)

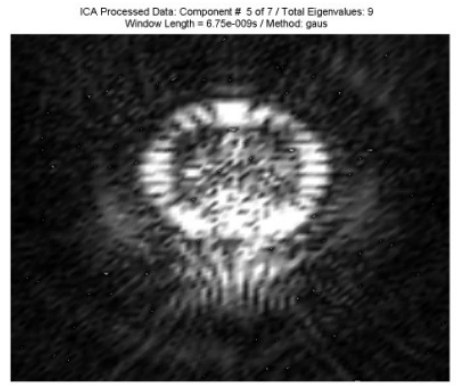

(e)

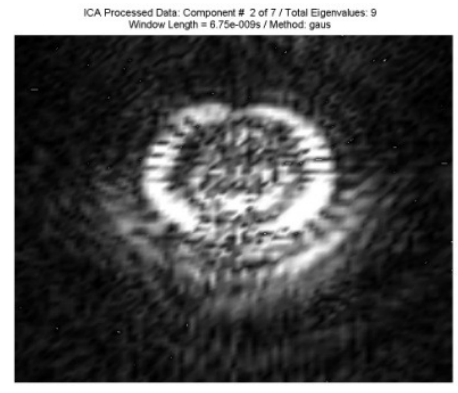

(b)

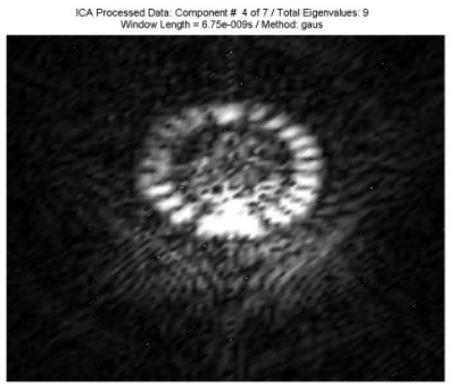

(d)

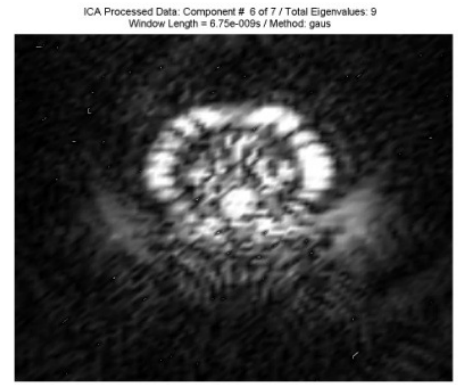

(f)

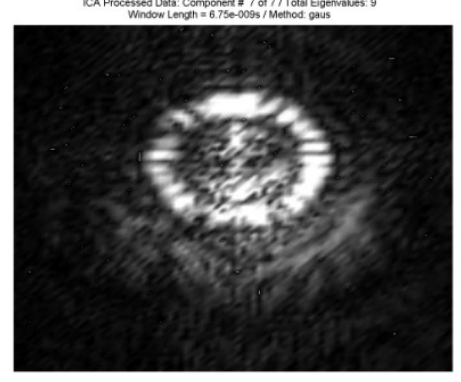

(g)

Figure 4-17 ICA results using symmetric orthogonalization (a) component \#1 (b) component \#2 (c) component \#3 (d) component \#4 (e) component \#5 (f) component \#6 (g) component \#7 


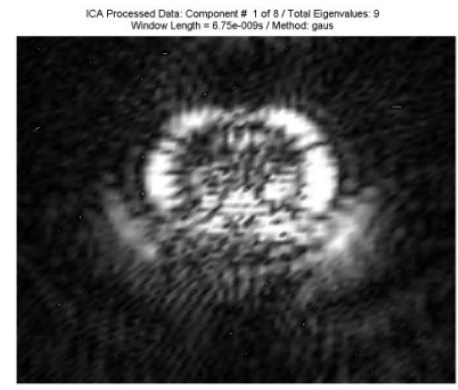

(a)

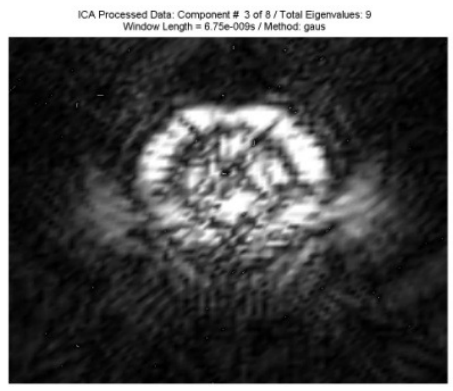

(c)

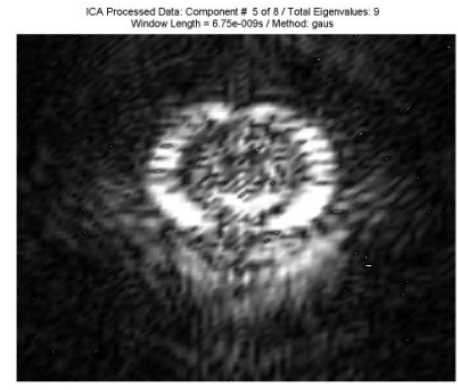

(e)

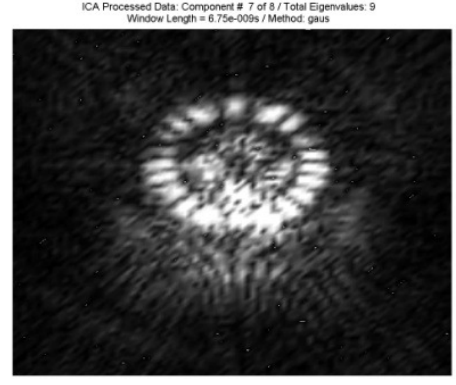

(g)

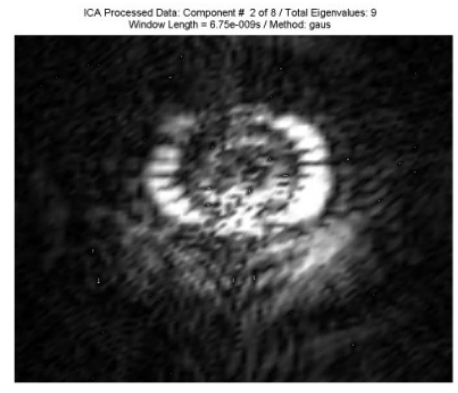

(b)

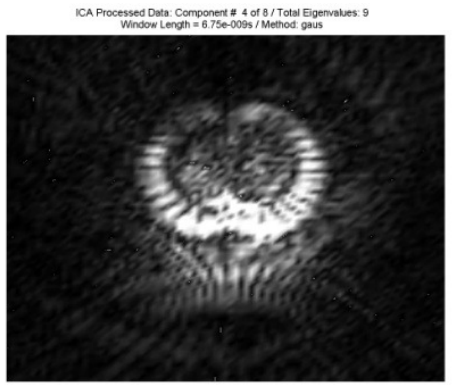

(d)

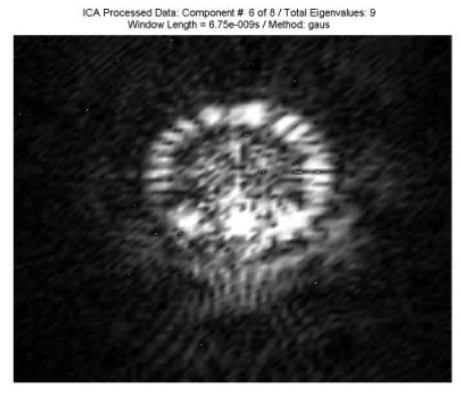

(f)

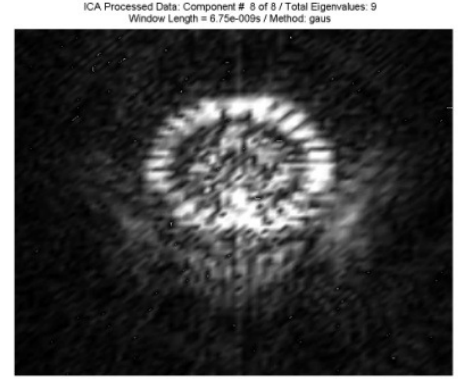

(h)

Figure 4-18 ICA results using deflation orthogonalization (a) component \#1 (b) component \#2 (c) component \#3 (d) component \#4 (e) component \#5 (f) component \#6 (g) component \#7 (h) component \#8 


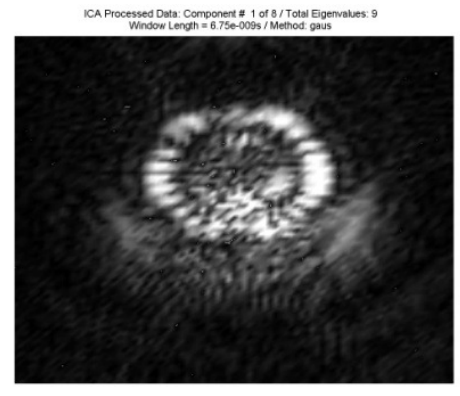

(a)

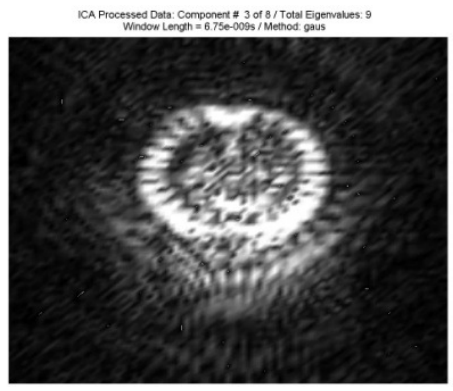

(c)

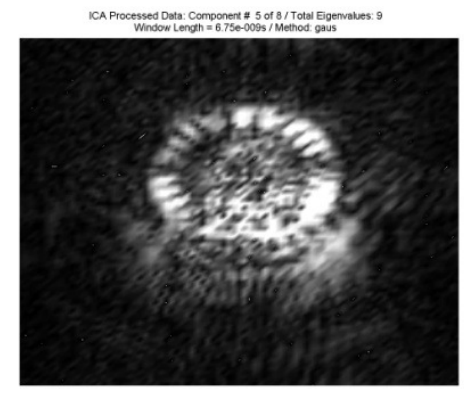

(e)

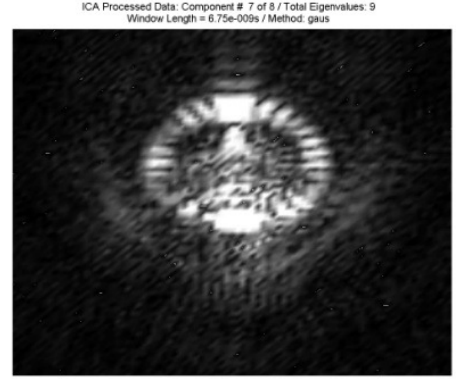

(g)

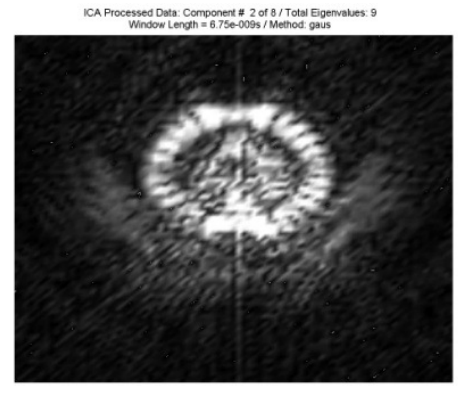

(b)

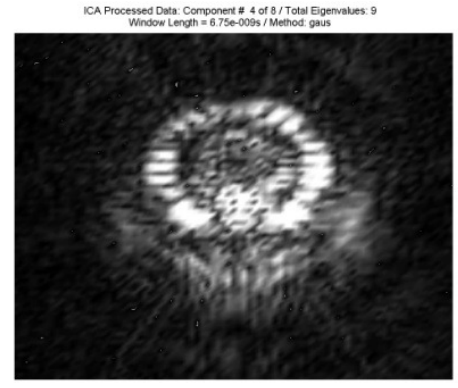

(d)

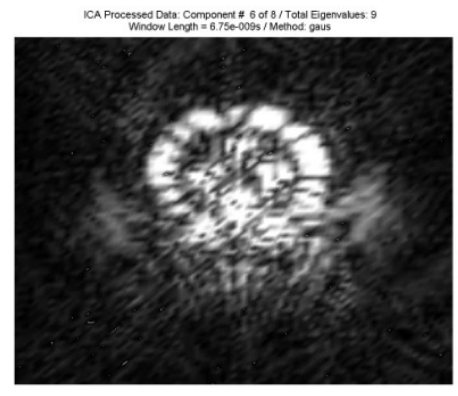

(f)

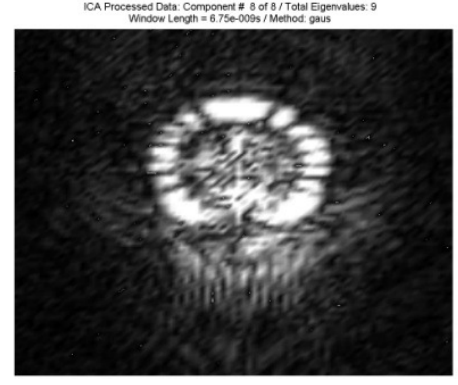

(h)

Figure 4-19 ICA results using symmetric orthogonalization (a) component \#1 (b) component \#2 (c) component \#3 (d) component \#4 (e) component \#5 (f) component \#6 (g) component \#7 (h) component \#8 


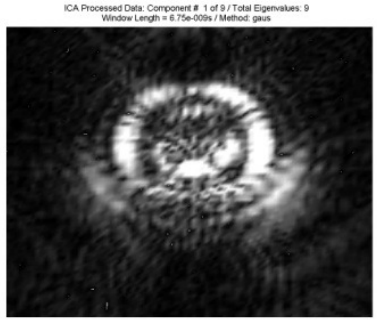

(a)

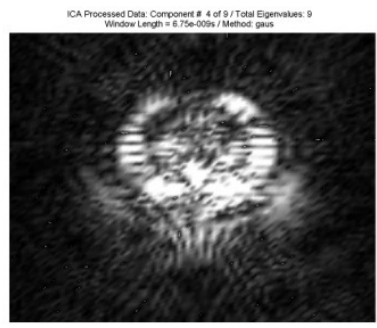

(d)

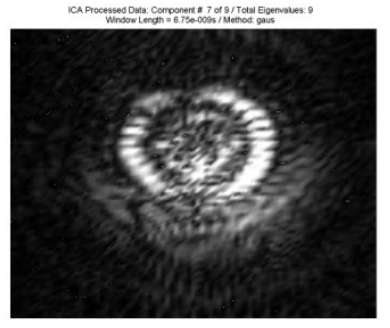

(g)

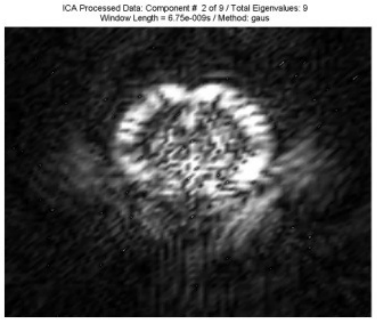

(b)

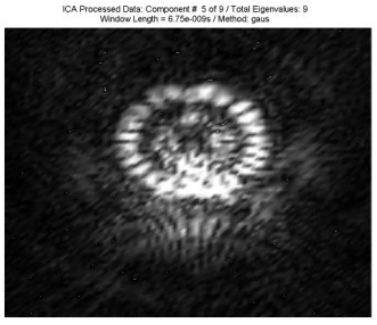

(e)

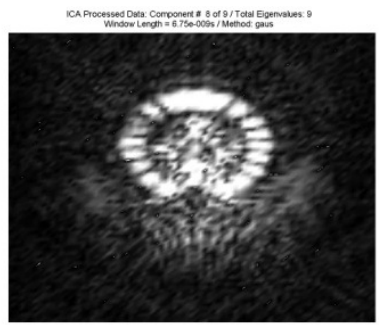

(h)

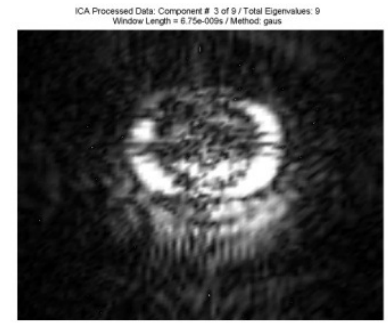

(c)

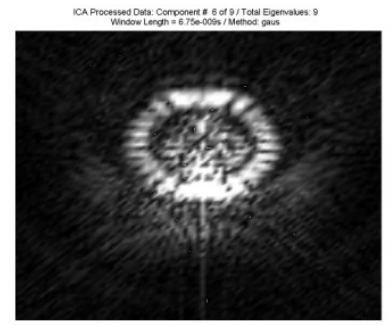

(f)

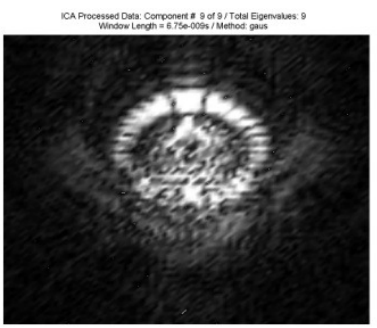

(i)

Figure 4-20 ICA results using deflation orthogonalization (a) component \#1 (b) component \#2 (c) component \#3 (d) component \#4 (e) component \#5 (f) component \#6 (g) component \#7 (h) component \#8 (i) component \#9 


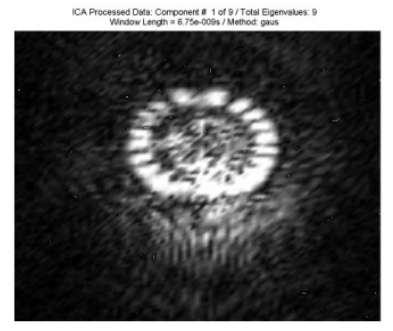

(a)

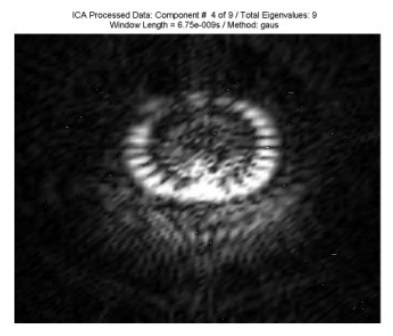

(d)

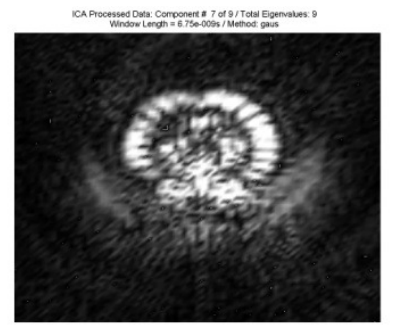

(g)

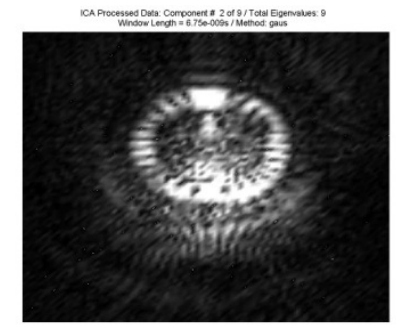

(b)

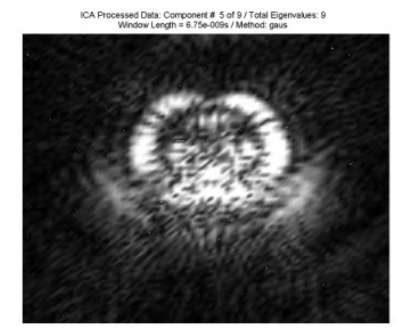

(e)

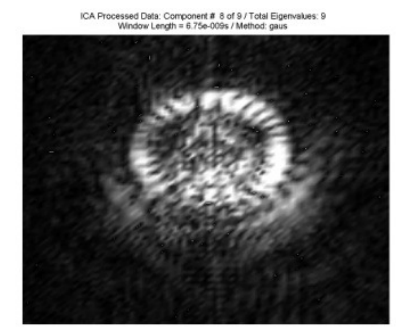

(h)

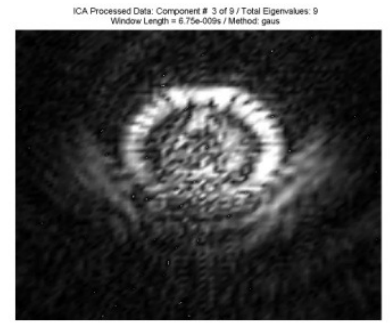

(c)

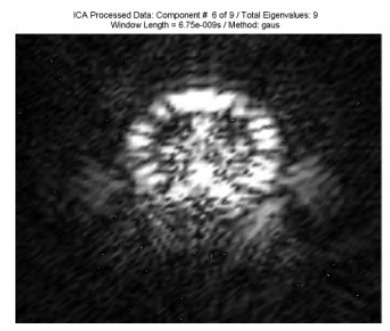

(f)

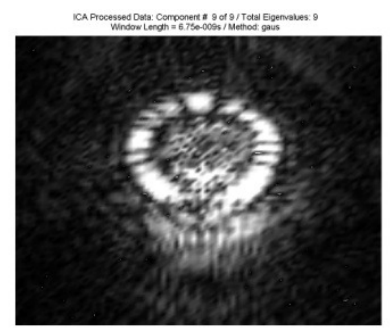

(i)

Figure 4-21 ICA results using symmetric orthogonalization (a) component \#1 (b) component \#2 (c) component \#3 (d) component \#4 (e) component \#5 (f) component \#6 (g) component \#7 (h) component \#8 (i) component \#9

xlt can be seen from the above images that the symmetric and deflationary approaches to orthogonalization of the independent components produce similar components however the symmetric approach appears to produce a higher level of detail in the separated components. It is known that the deflationary approach tends to propagate orthogonalization errors and henceforth produces a smearing of the independent components. Given the qualitative empirical proof as well as the stated theoretical basis the remainder of this study will employ the symmetric orthogonalization methodology.

\subsection{Whitening Matrix Dimension Selection}

The next parameter examined is the selection of the number of eigenvalues retained from the PCA preprocessing to be used in the whitening of the data. The dimension of the data 
whitening matrix is limited by the number of eigenvalues selected for retention from the PCA preprocessing step. For guidance in selection of this parameter a metric produced by the FastlCA algorithm was used. This metric was namely the percent of non-zero eigenvalues retained. The metric is derived from a ratio of the sum of the selected eigenvalues to the total eigenvalues of the data. This metric was examined for multiple data sets and a qualitative threshold for the metric was selected as $90 \%$. Figure $4-22$ shows the variation of this metric over the selected number of eigenvalues. Using the FastICA algorithm it is possible to specify the index of the largest eigenvalue to retain and thus extract a subset of smaller eigenvalues for use in the whitening process. For purposes of examination of this parameter it was assumed that the largest eigenvalues were always retained. To show the effect of the number of eigenvalues retained on the information extracted, the independent components for representative samples above and below the $90 \%$ threshold are shown. For the offset 6 " sphere dataset the $90 \%$ threshold was met by the retention of nine eigenvalues as can be seen in Figure 4-22. Due to the fact that the maximum number of independent components which can be extracted is limited by the dimension of the whitened data which is in turn limited by the number of eigenvalues used in the whitening process, the lesser case will show fewer independent components. This limitation is shown as the range of selection parameters for the independent components in Table 4-1. For the greater case, the number of independent components will be fixed at nine to enable comparison with the $90 \%$ case. 


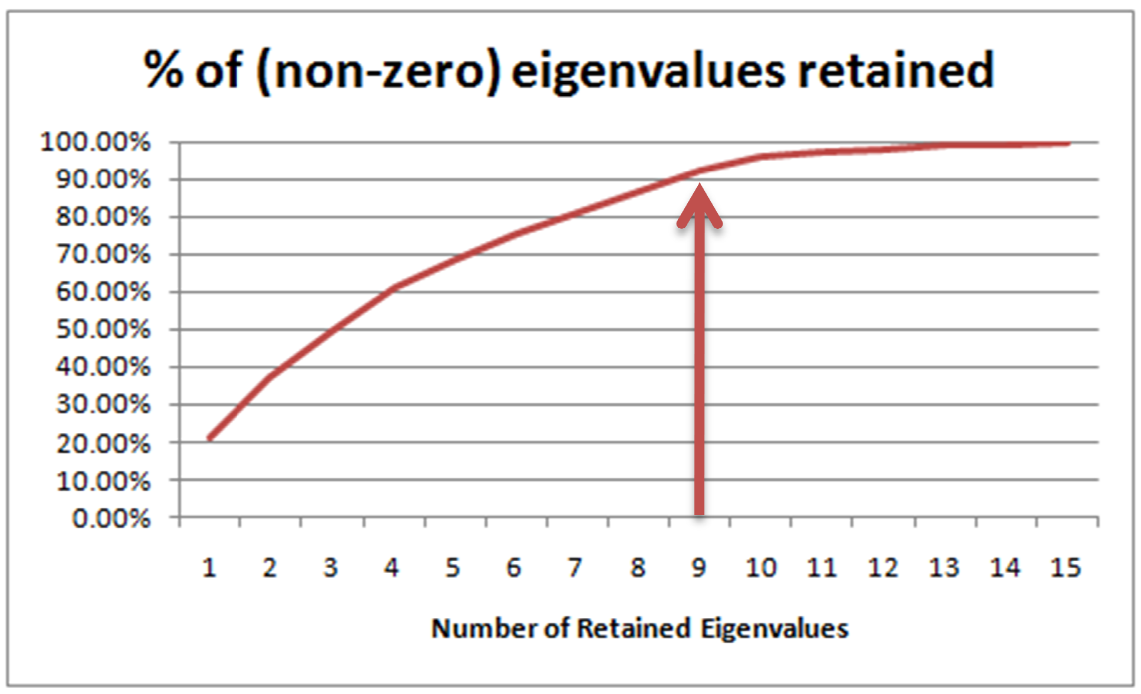

Figure 4-22 Eigenvalue retention metric for offset 6" sphere 
ICA Processed Data: Component \# 1 of 2 / Total Eigenvalues: 2 Window Length $=6.75 \mathrm{e}-009 \mathrm{~s} /$ Method: gaus

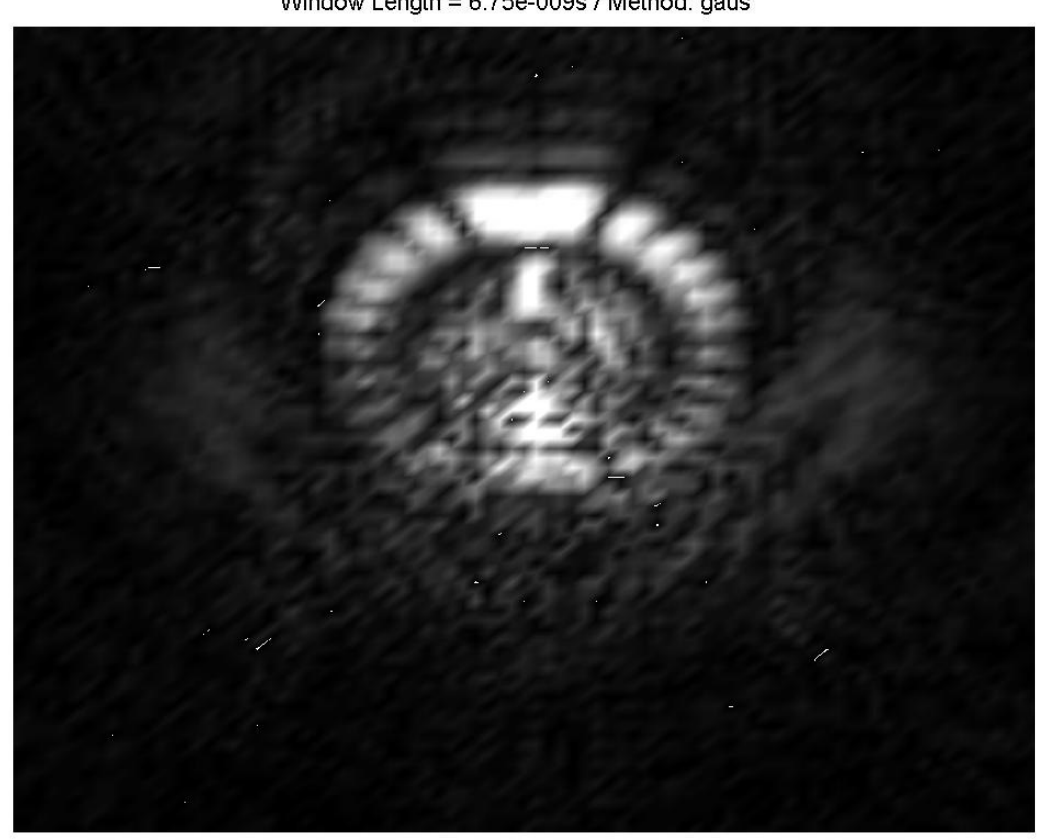

(a)

ICA Processed Data: Component \# 2 of 2 / Total Eigenvalues: 2

Window Length $=6.75 \mathrm{e}-009 \mathrm{~s} /$ Method: gaus

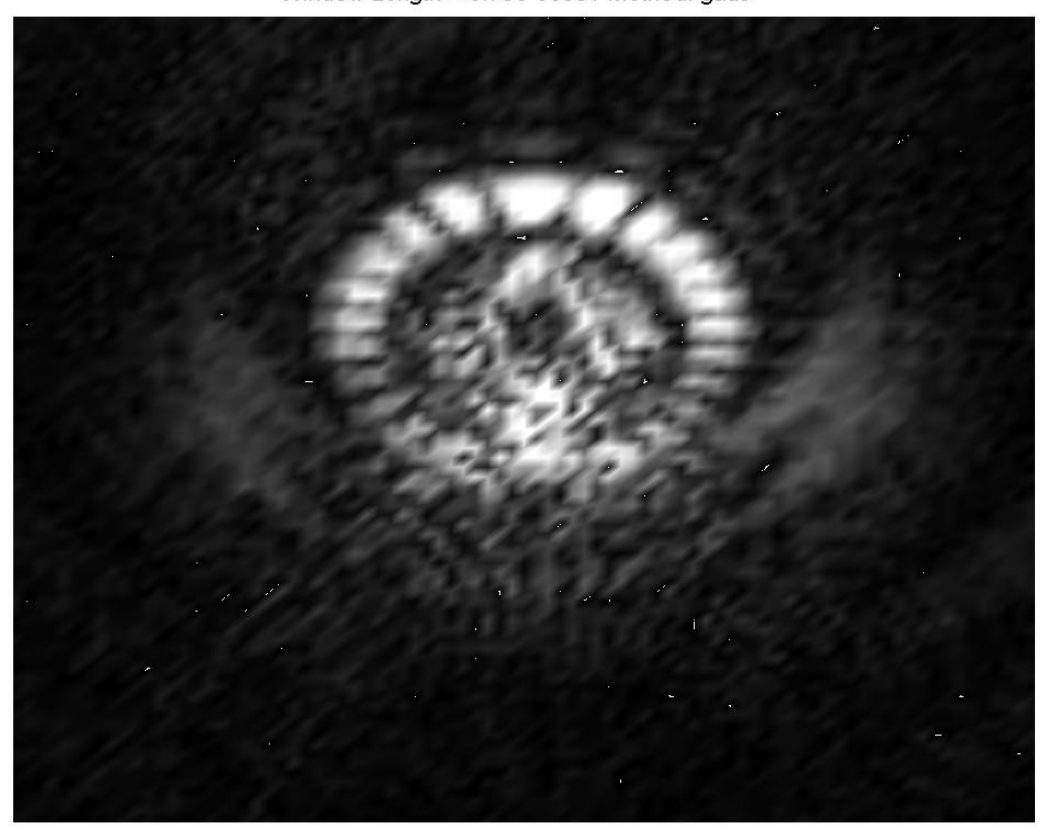

(b)

Figure 4-23 ICA results with 2 ICs and 2 EVs (a) component \#1 (b) component \#2 


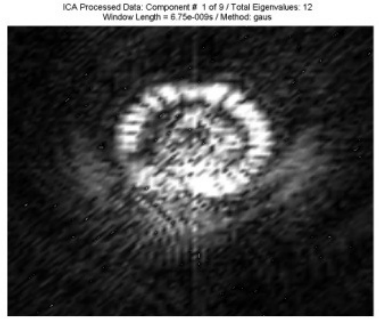

(a)

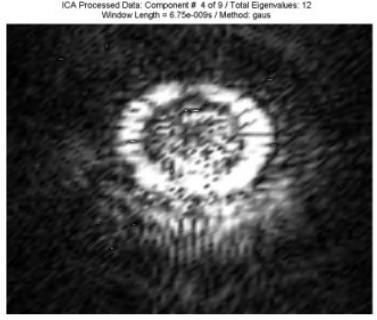

(d)

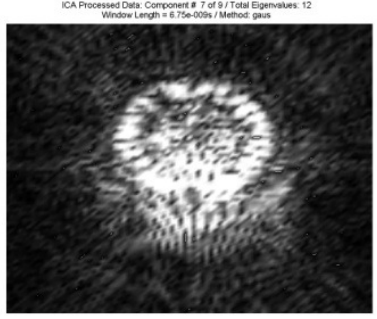

(g)

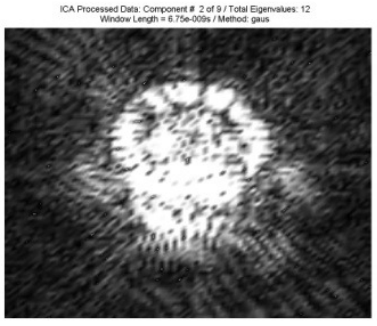

(b)

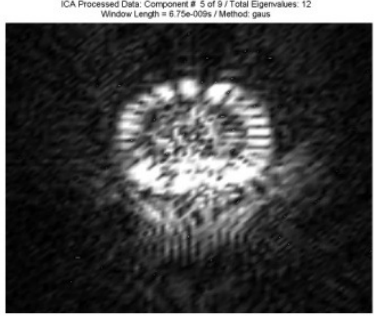

(e)

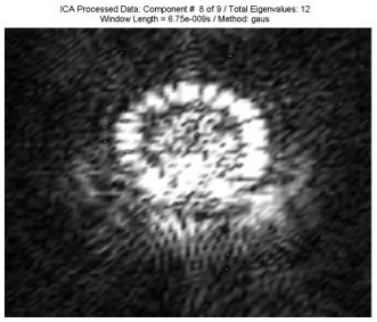

(h)

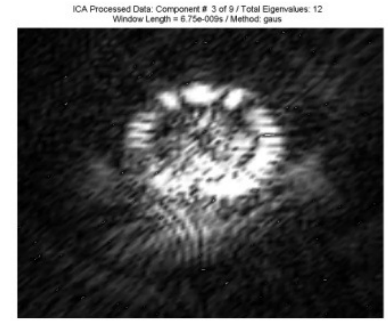

(c)

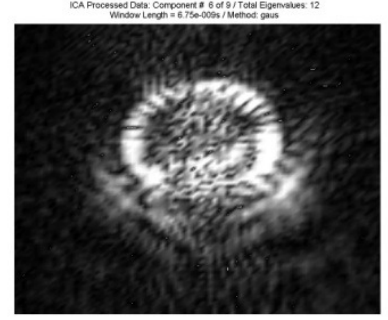

(f)

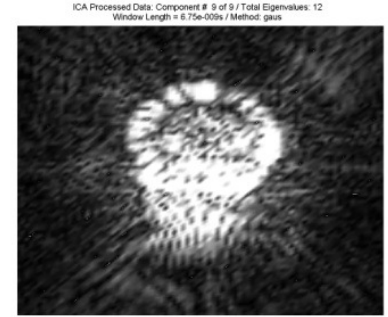

(i)

Figure 4-24 ICA results with 9 ICs and 12 EVs (a) component \#1 (b) component \#2 (c) component \#3 (d) component \#4 (e) component \#5 (f) component \#6 (g) component \#7 (h) component \#8 (i) component \#9 


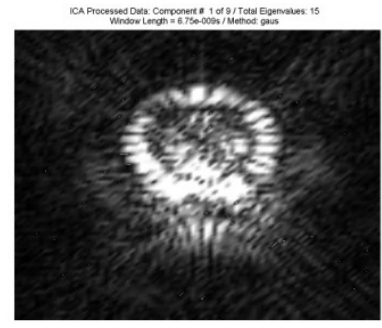

(a)

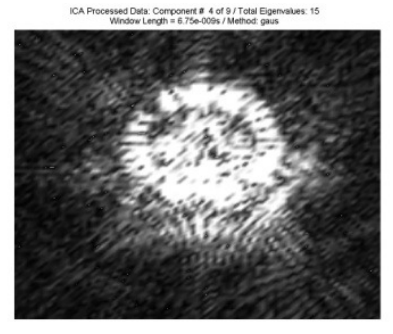

(d)

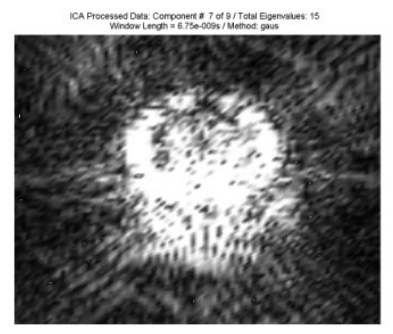

(g)

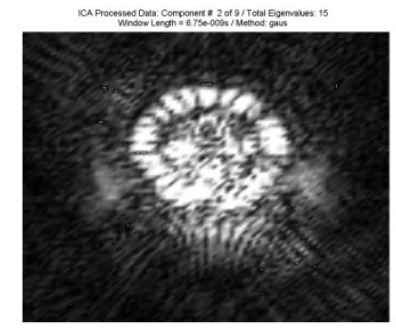

(b)

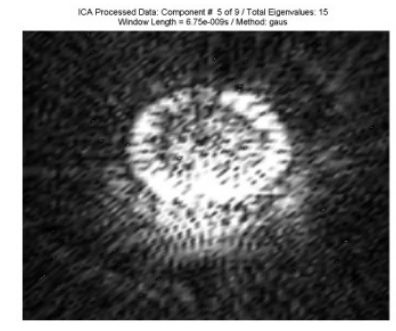

(e)

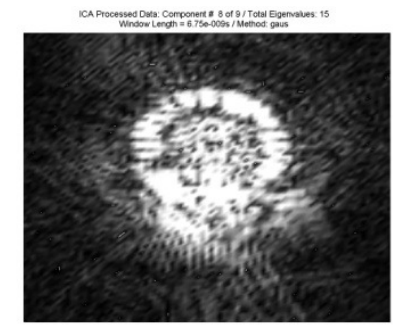

(h)

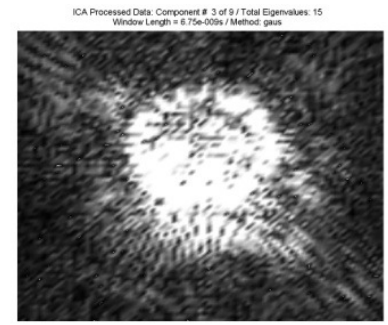

(c)

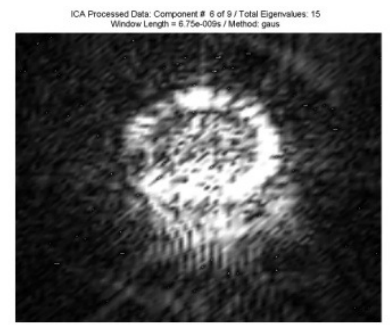

(f)

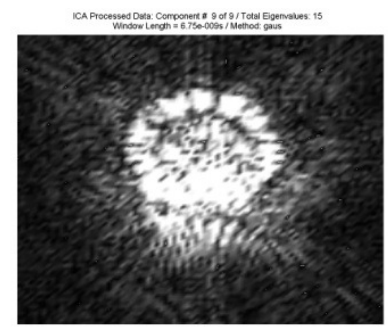

(i)

Figure 4-25 ICA results with 9 ICs and 15 EVs (a) component \#1 (b) component \#2 (c) component \#3 (d) component \#4 (e) component \#5 (f) component \#6 (g) component \#7 (h) component \#8 (i) component \#9

For the lesser case the number of eigenvalues retained was set to two which corresponds to the metric value $37.38 \%$. It can be seen, by comparing Figure $4-23$ with Figure 4-21, that a greater number of eigenvalues allows the whitening matrix to more correctly decorrelate the underlying sources. As a result of the lesser decorrelation due to the small number of eigenvalues retained in Figure 4-23 the underlying sources are blended as the whitening has produce decorrelation for only two of the underlying components. Thus a larger number of retained eigenvalues is desired to enable accurate decorrelation of the underlying components in the mixture. There is a limit to the benefit of increasing the dimension of the whitening matrix. This limit comes as the whitening matrix begins to decorrelate noise-like signals within the mixture. The effects of this are illustrated in Figure 4-24 and Figure 4-25. These figures 
show the nine extracted independent components for twelve and fifteen retained eigenvalues respectively. The retention of twelve eigenvalues corresponds to the metric value of $98.11 \%$ and 15 corresponds to $99.54 \%$. It can be seen that some of the details of the images of the extracted components begin to be obscured by what appears to be a noise-like component. Through this qualitative assessment a case has been made to guide the choice of the eigenvalue retention parameter selection. The remainder of this study will present results which adhere to the $90 \%$ non-zero eigenvalues retention.

\subsection{Independent Component Quantity Parameter Selection}

As shown in Table 4-1, the number of independent components which can be estimated is limited by the dimension of the whitening matrix. To date there is not a method of relating scattering properties to independent components therefore the conservative approach for selection of this parameter was to maximize the number of components selected. This approach ensures that the set of underlying components is not unnecessarily truncated. The effect of gradual increase of the independent component quantity parameter under the constraint of a dimension nine whitening matrix can be seen in Figure 4-4 through Figure 4-21. The remainder of this thesis will present results which correspond to maximum number independent components that can be extracted for a set of target data. This number is inextricably linked to the dimension of the whitening matrix which is derived from the number of retained eigenvalues which for this study is controlled by the $90 \%$ threshold.

\subsection{Nonlinear Estimator Parameter Selection}

The final parameter being considered in the development of this study is the choice of the nonlinear estimator used in examining the non-gaussianity of the components of the mixture. The available choices along with their corresponding formulas can be seen in Figure 2-4. The figure below illustrates the performance variation of the different estimators. The cases shown in Figure 4-26 are for a size nine whitening matrix and 3 independent components. The choice of 3 ICs was purely for easy of presentation of the information. A qualitative assessment of the results, produced by the different estimators, shows that the "gaus" estimator appears to produce finer detail in the extracted components. This detail can be seen in the "lobing" structure about the circumference of the sphere. The other methods produced extracted components in which much 
of this detail was smeared. Throughout the remained of this thesis a similar qualitative analysis was performed and the results of the will be shown using only the "best" performing estimator. Thusly the independent components, of the offset 6 " sphere, produced using the above developed rational can be seen in Figure 4-21. 


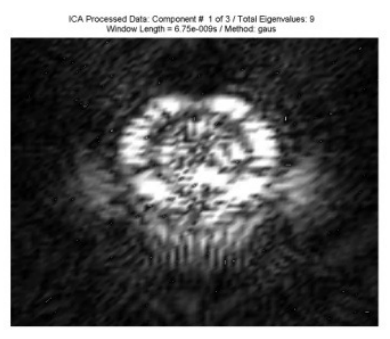

(a)

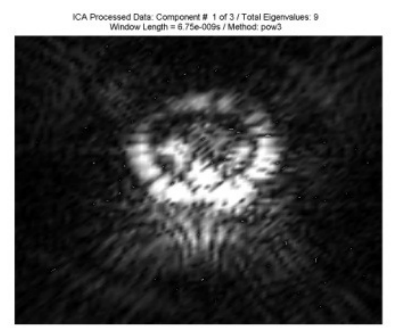

(d)

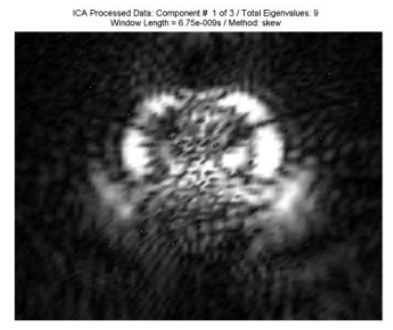

(g)

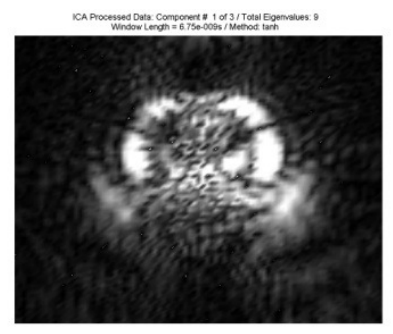

(j)

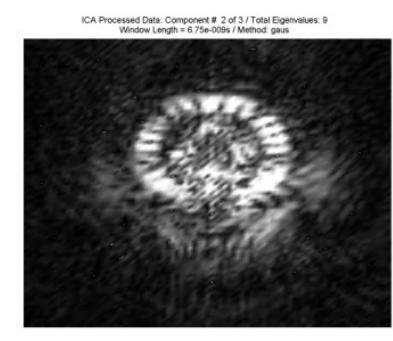

(b)

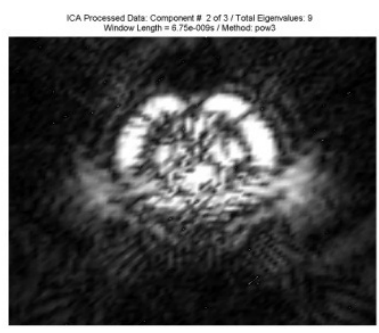

(e)

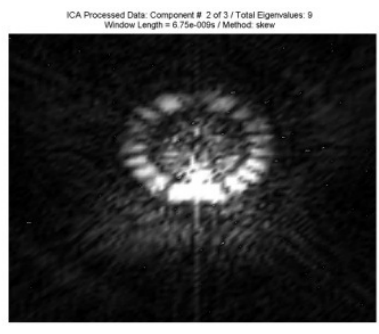

(h)

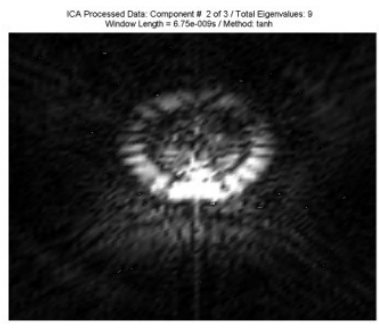

(k)

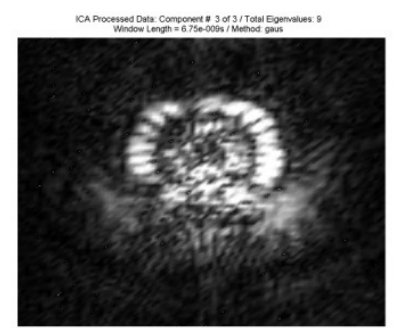

(c)

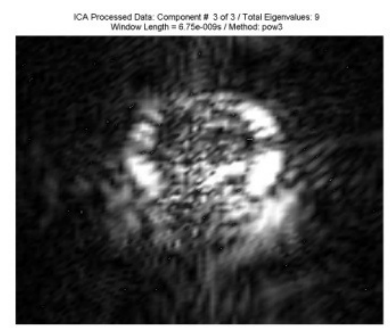

(f)

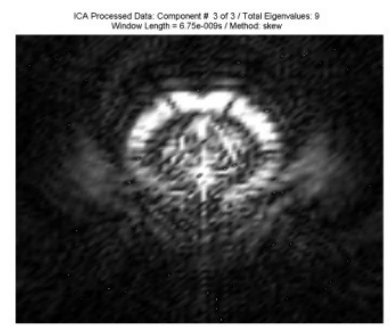

(i)

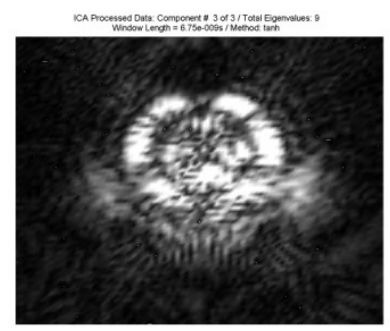

(I)

Figure 4-26 Nonlinear estimator comparison (a) gaus: component \#1 (b) gaus: component \#2 (c) gaus: component \#3, (d) pow3: component \#1 (e) pow3: component \#2 (f) pow3: component \#3 (g) skew: component \#1 (h) skew: component \#2 (i) skew: component \#3 (j) tanh: component \#1 (k) tanh: component \#2 (I) tanh: component \#3 


\subsection{Additional Data Sets}

The next portion of this paper will present the results of application of the FastlCA algorithm to several different data sets. The extracted components of each data set will be shown under the constraint of the algorithm option selection developed above. Each data set will include a photo of the target and a plot of the eigenvalue retention metric used above.

\subsubsection{Offset 4" Sphere}

This target is a 4" sphere offset from the center of the turntable by $~ 4 "$. An image of the target can be seen in Figure 4-27. From the eigenvalue retention metric, shown in Figure 4-29, it can be seen that eight eigenvalues are used in generating the whitening matrix which also implies that the number of independent components to be extracted is also eight.

An ISAR image of the original data as well as the time gated data is shown in Figure 4-28. The next set of images shows the results of the ICA extraction using the "gaus" nonlinear estimator. The results can be seen in Figure 4-30 (a) - (h).

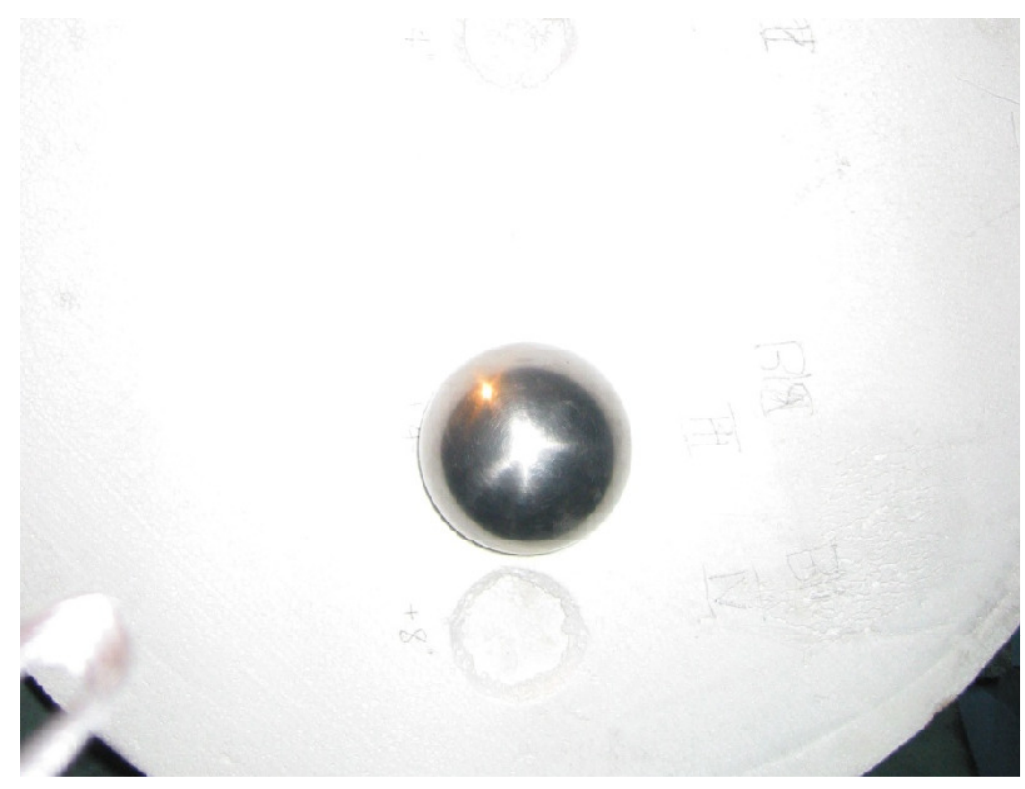

Figure 4-27 Photo of offset 4" sphere 


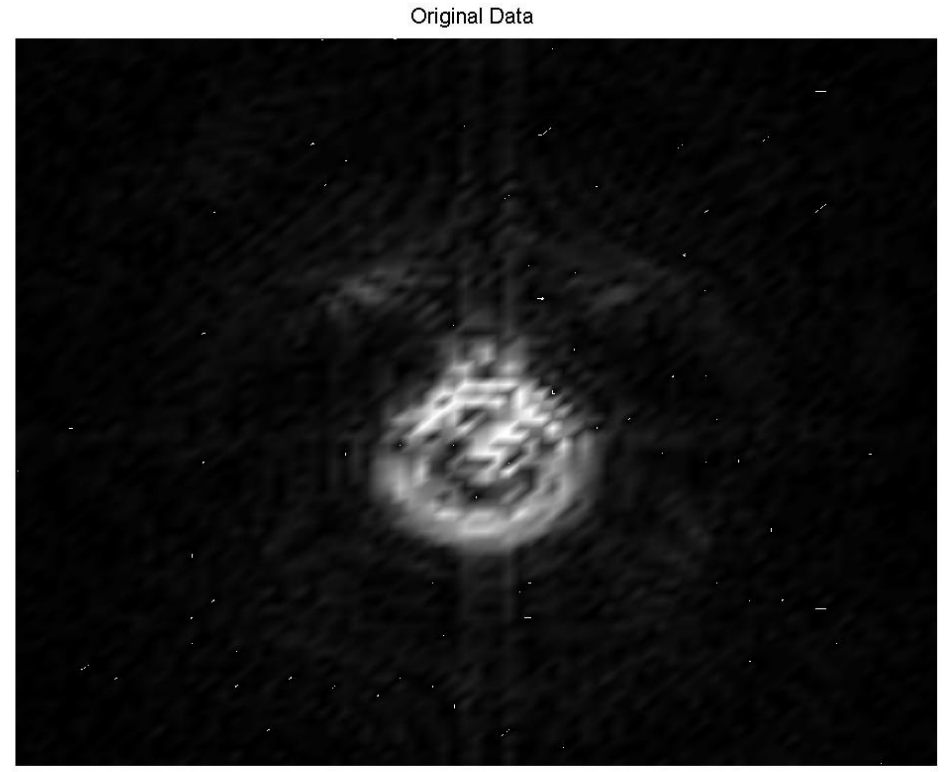

(a)

Time Windowed Data : Window Length $=6.75 \mathrm{e}-009 \mathrm{~s}$

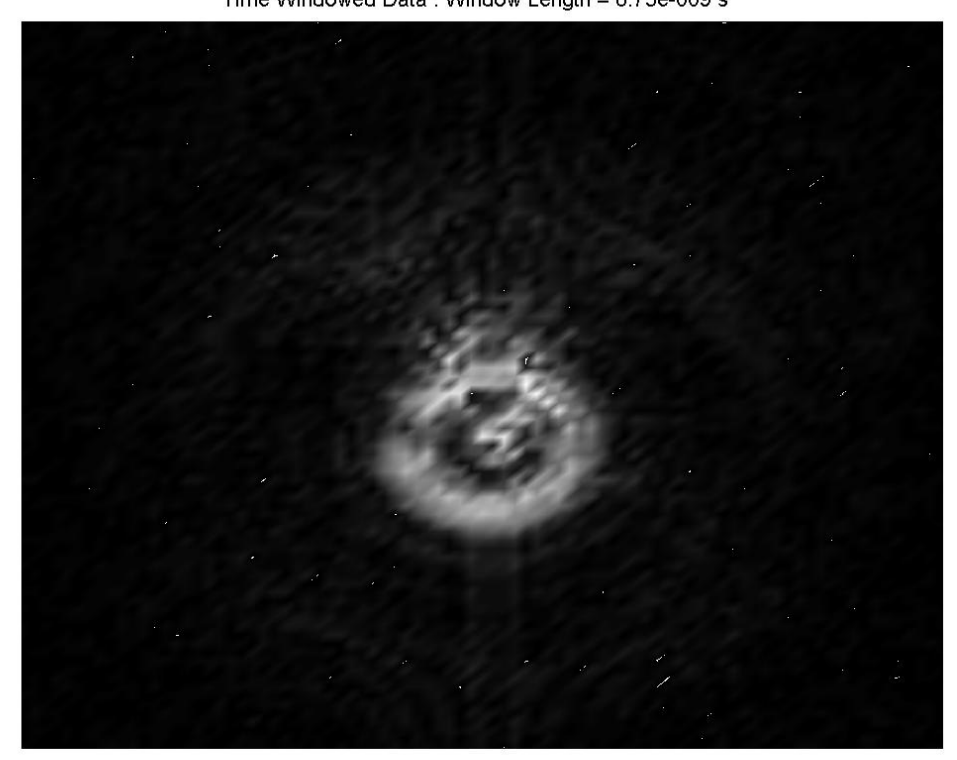

(b)

Figure 4-28 (a) ISAR image formed with raw scattering data (b) ISAR image formed from timegated scattering data 


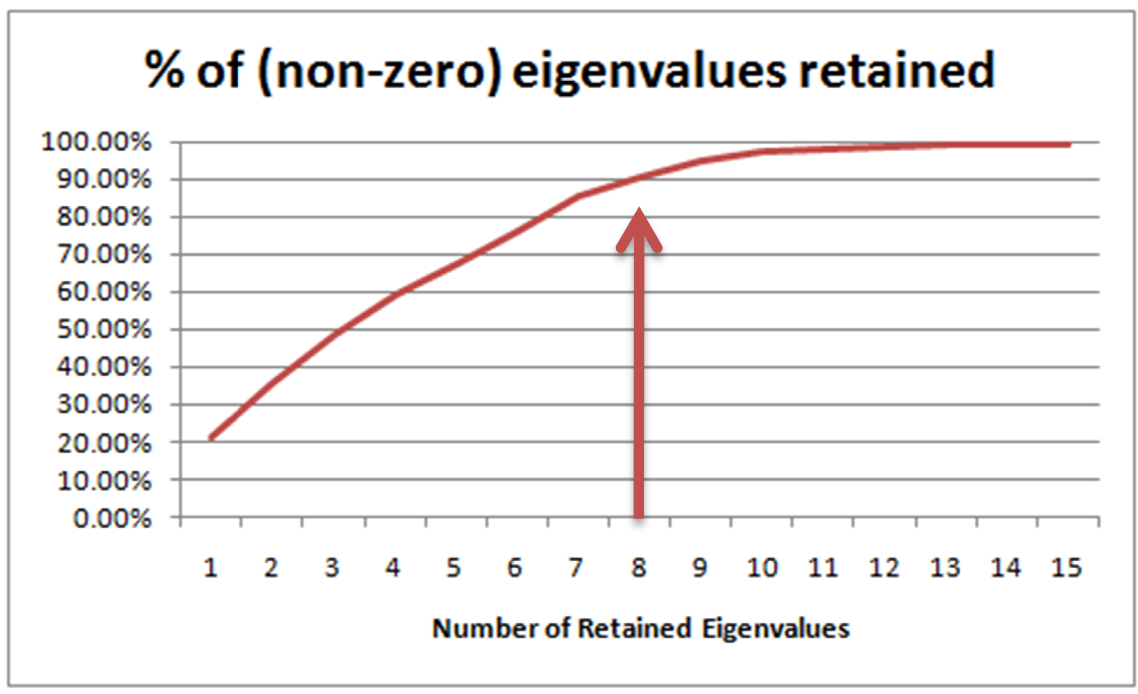

Figure 4-29 Eigenvalue retention metric for offset 4" sphere 


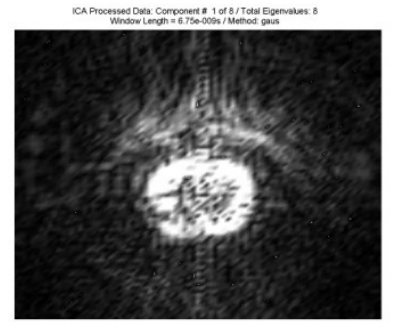

(a)

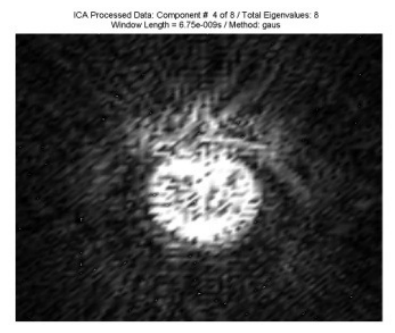

(d)

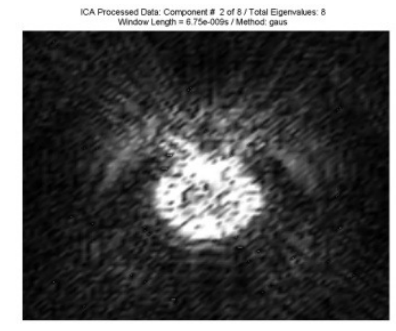

(b)

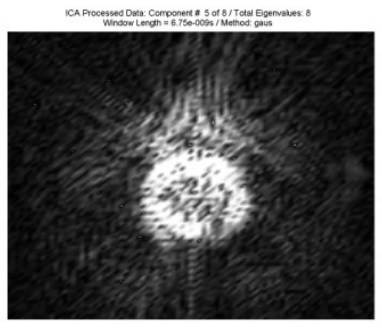

(e)

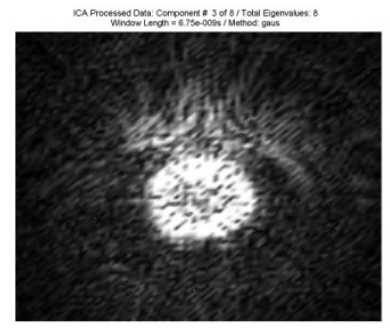

(c)

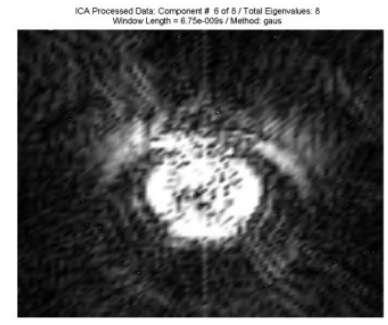

(f)

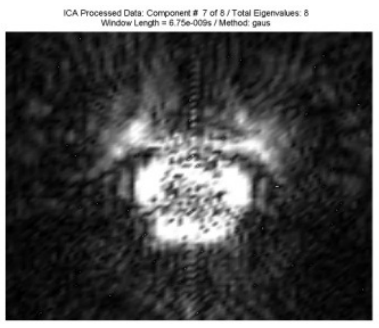

(g)

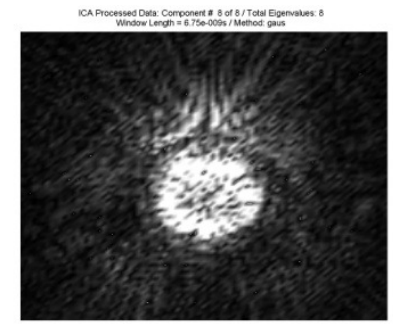

(h)

Figure 4-30 ICA results with eight ICs and eight EVs (a) component \#1 (b) component \#2 (c) component \#3 (d) component \#4 (e) component \#5 (f) component \#6 (g) component \#7 (h) component \#8

\subsubsection{Four Inch Sphere and Six Inch Sphere: 8" Separation}

The next target examined increases the number of scattering mechanisms by placing two reflective spheres in close proximity to one another. The spheres are placed approximately 8" center to center. It is expected that an additional multi-bounce interaction echo as depicted in Figure 2-2 should be present. This target set begins to venture from the well controlled laboratory target of a single scatterer to one more representative of a practical target.

An image of the target set can be seen in Figure 4-31 and the target's metric is shown in Figure 4-33. It can be seen that the eigenvalues and the independent components were both set to eight. The nonlinear estimator used for this data set is "pow3." Again the reader is referred to 
Figure 2-4 for equations for the estimators. An ISAR image of the original data as well as the time-gated data is presented in Figure 4-32.

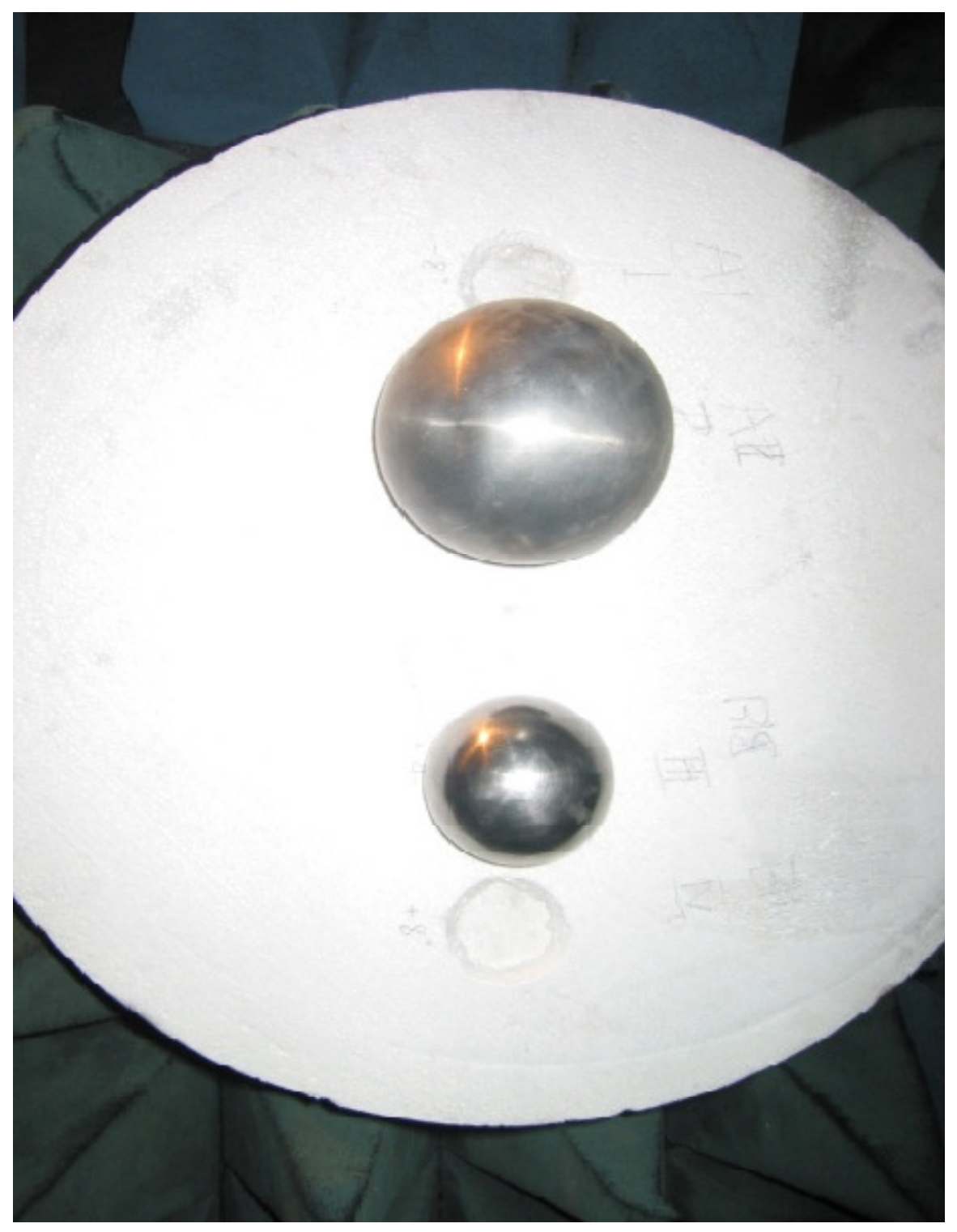

Figure 4-31 Photo of 4" sphere and 6" sphere: 8" separation 


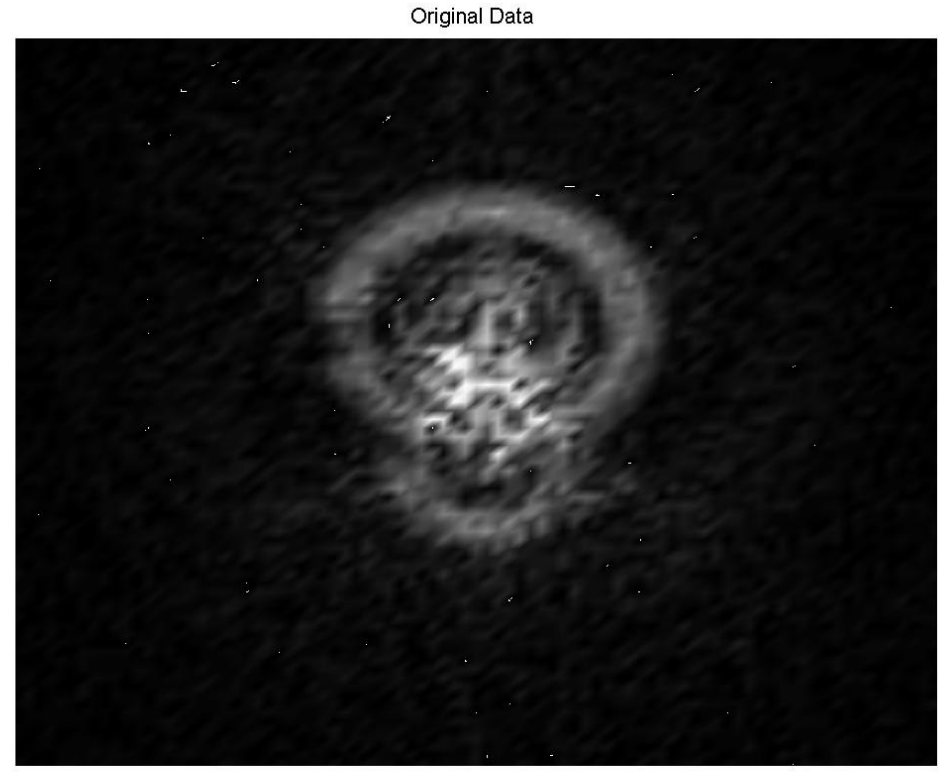

(a)

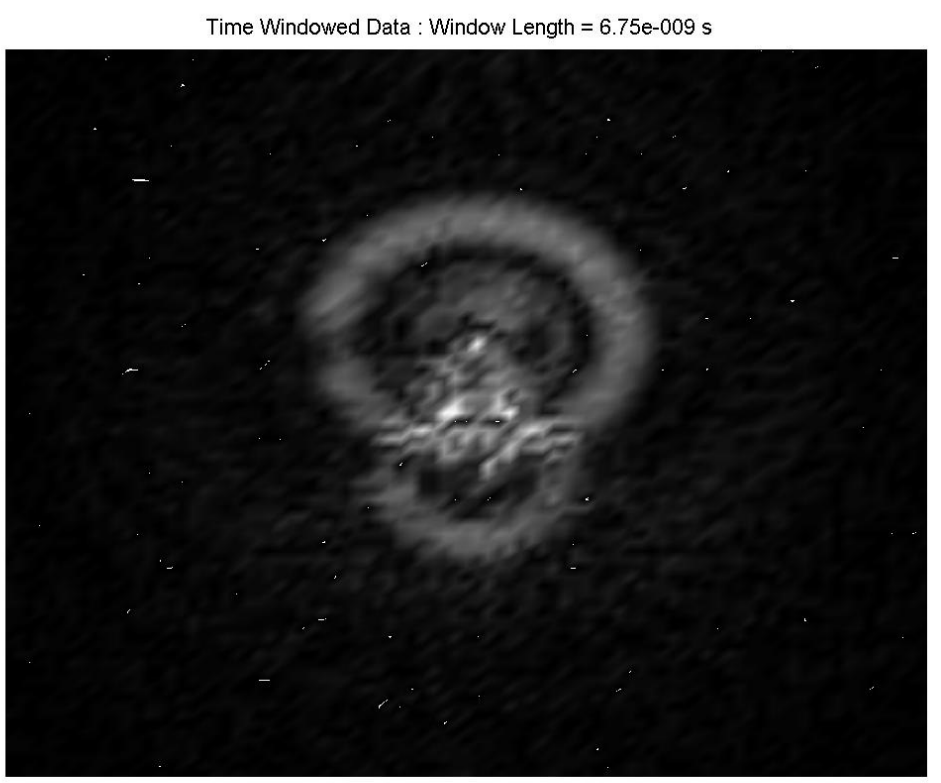

(b)

Figure 4-32 (a) ISAR image formed with raw scattering data (b) ISAR image formed from timegated scattering data 


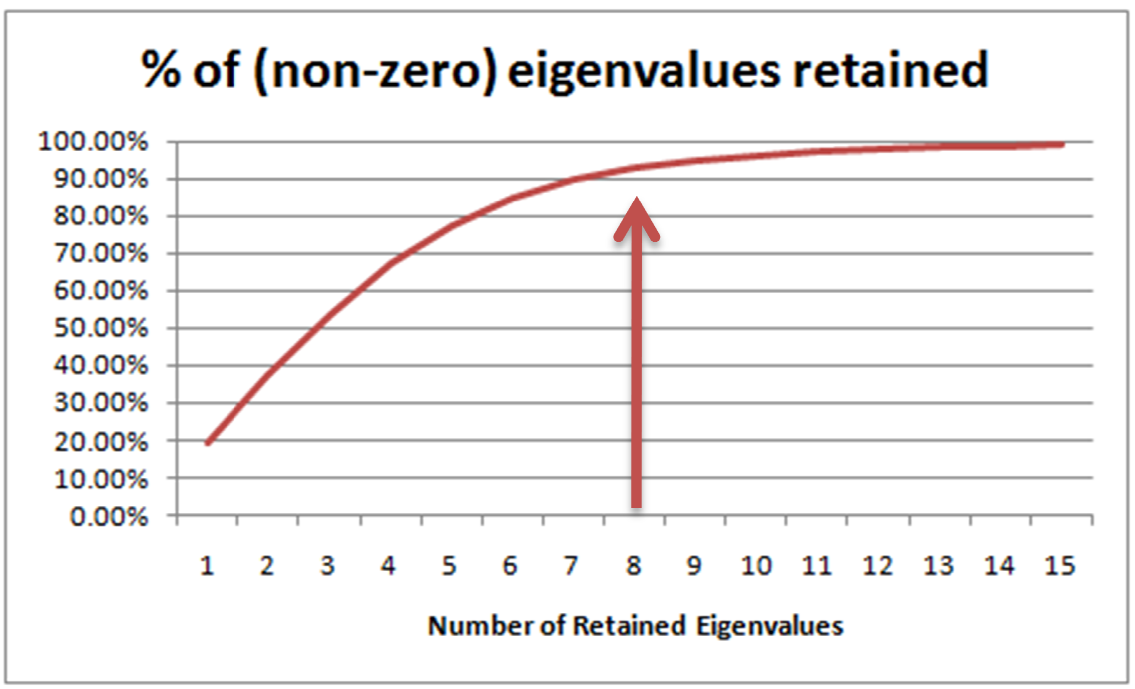

Figure 4-33 EV retention metric for 4" sphere and 6" sphere @ 8" separation 


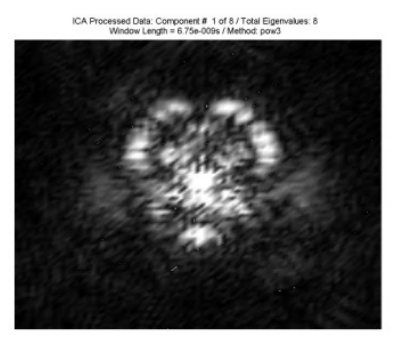

(a)

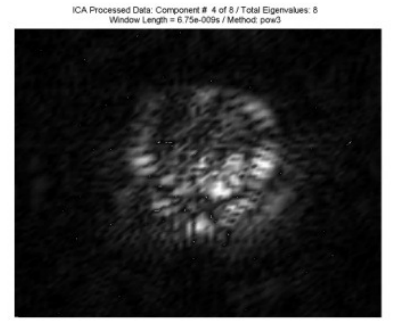

(d)

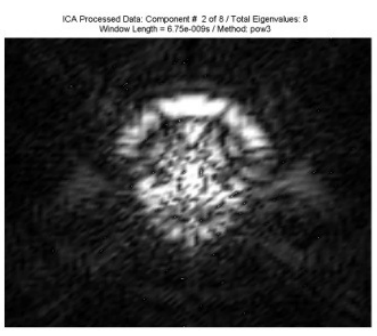

(b)

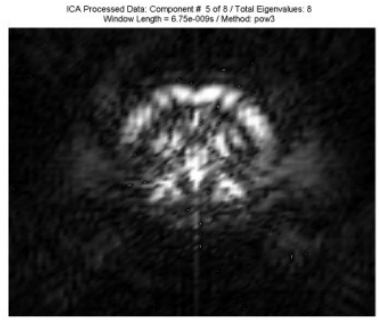

(e)

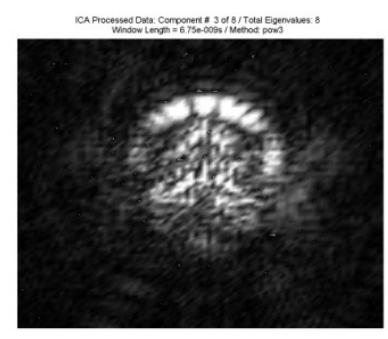

(c)

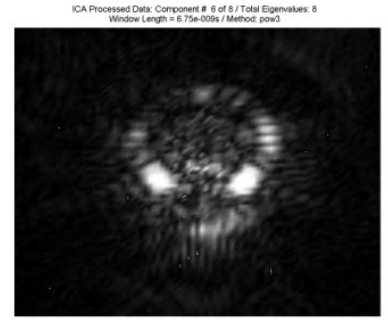

(f)

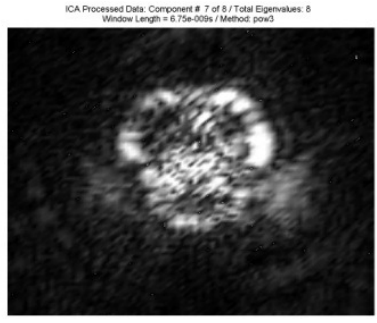

(g)

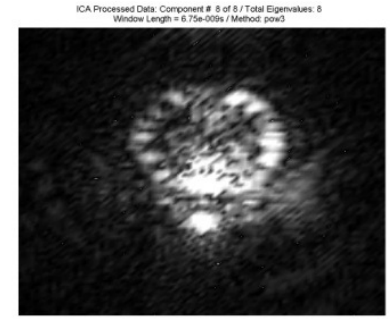

(h)

Figure 4-34 ICA results with eight ICs and eight EVs (a) component \#1 (b) component \#2 (c) component \#3 (d) component \#4 (e) component \#5 (f) component \#6 (g) component \#7 (h) component \#8

\subsubsection{Four Inch Sphere and Six Inch Sphere: 16" Separation}

This target also includes both the 6" and 4" spheres. The only difference between this target and the previous is the distance placed between the spheres. This target places approximately 16 " between the sphere centers.

An image of the target set can be seen in Figure 4-35 and the target's metric is shown in Figure 4-37. The results shown below will have the eigenvalues and the independent components both set to 11. The nonlinear estimator used for this data set is "pow3." The ISAR images of the original data and the time-gated data are presented in Figure 4-36. 


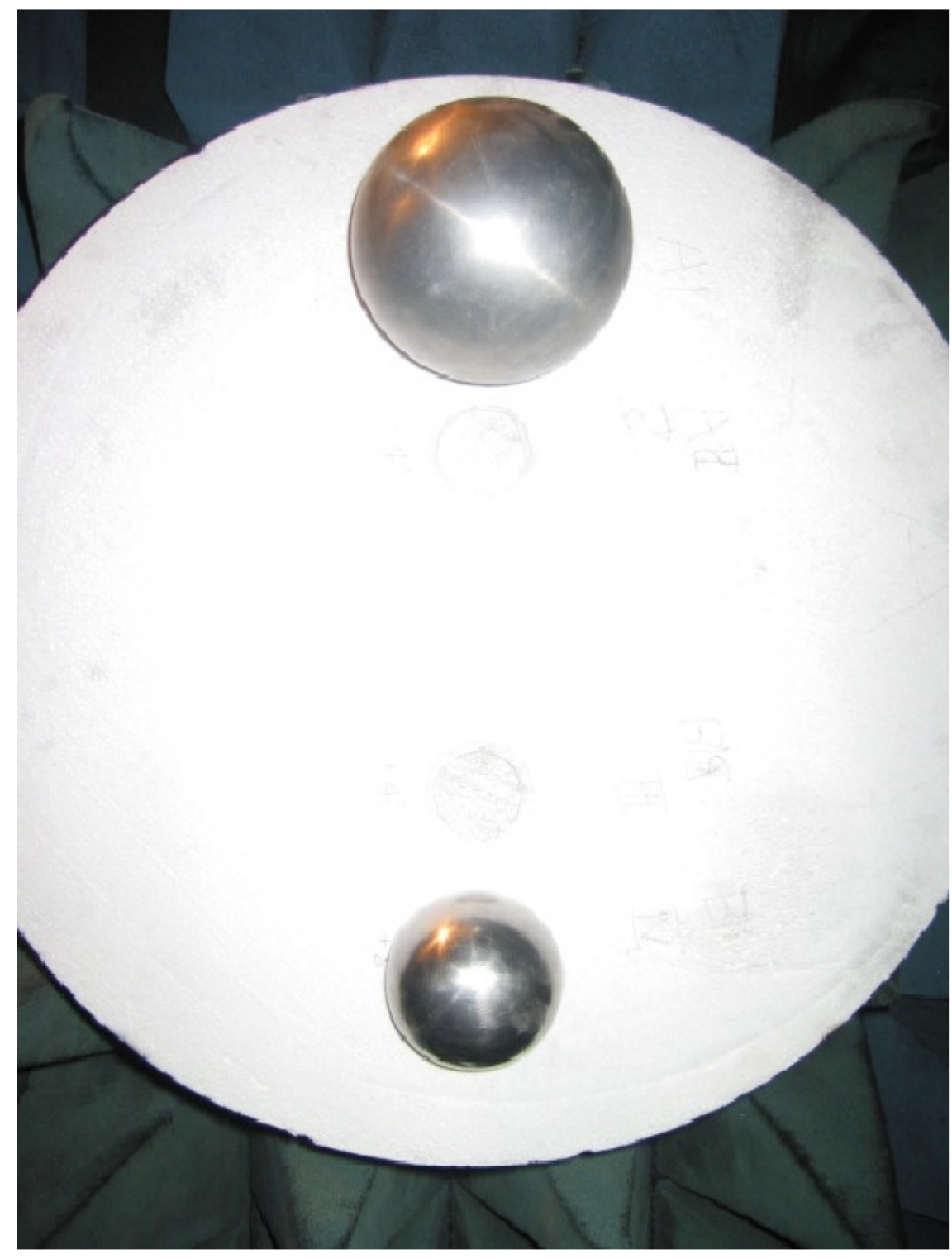

Figure 4-35 Photo of 4" sphere and 6" sphere: 16" separation 


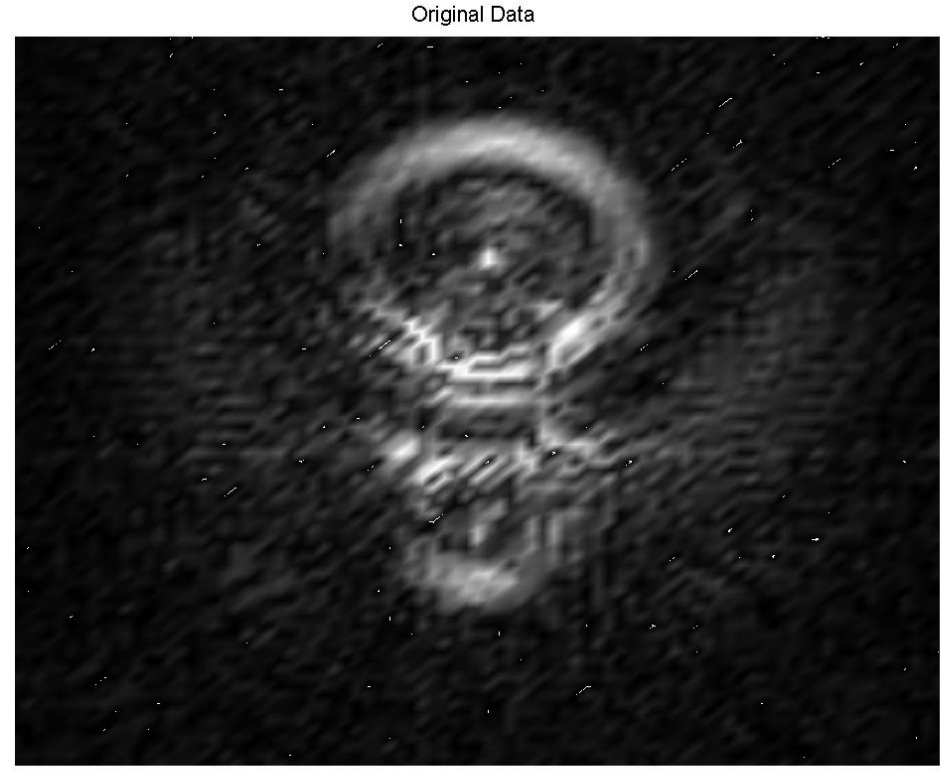

(a)

Time Windowed Data : Window Length $=6.75 \mathrm{e}-009 \mathrm{~s}$

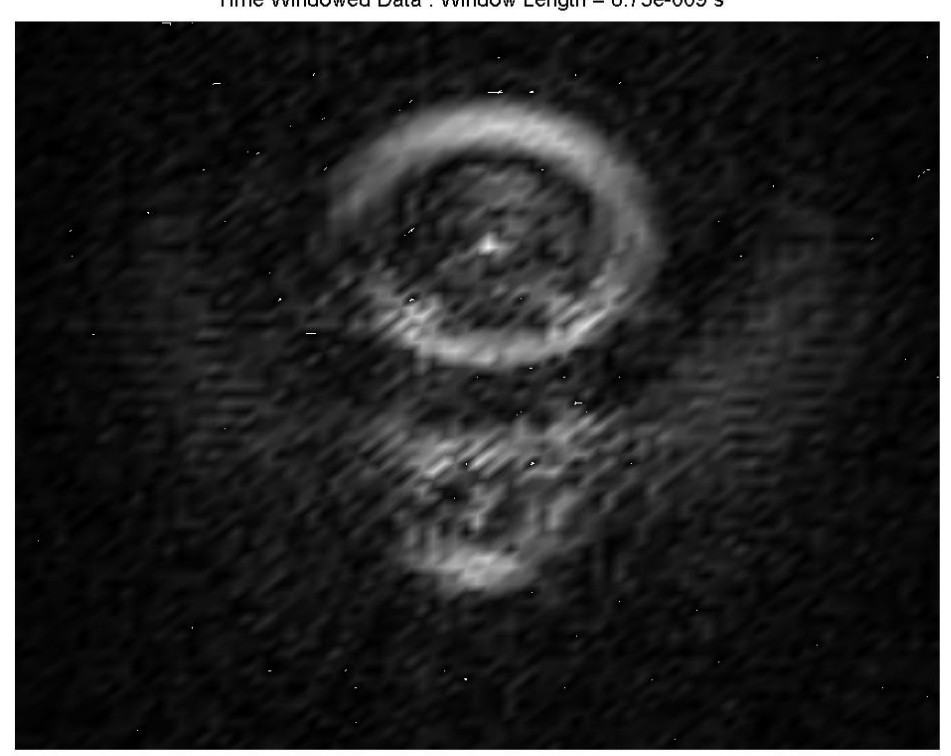

(b)

Figure 4-36 (a) ISAR image formed with raw scattering data (b) ISAR image formed from timegated scattering data 


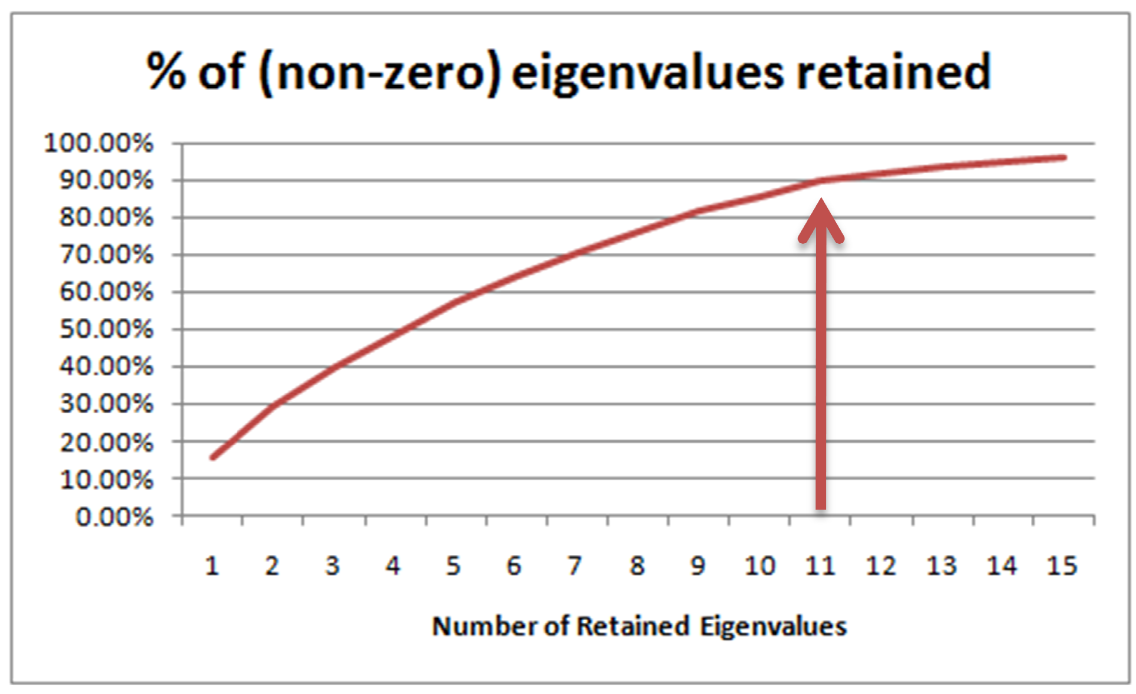

Figure 4-37 EV retention metric for 4" sphere and 6" sphere @ 16" separation 


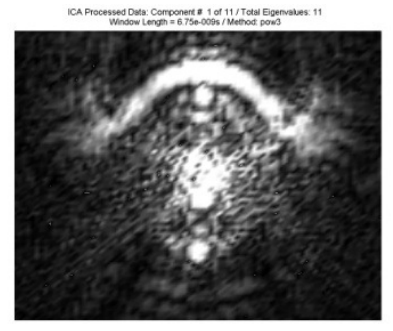

(a)

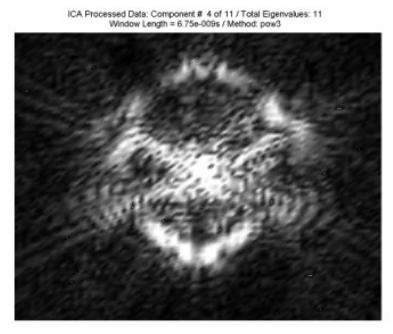

(d)

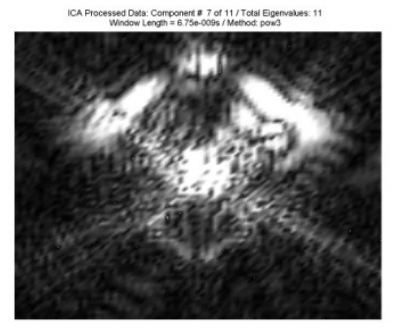

(g)

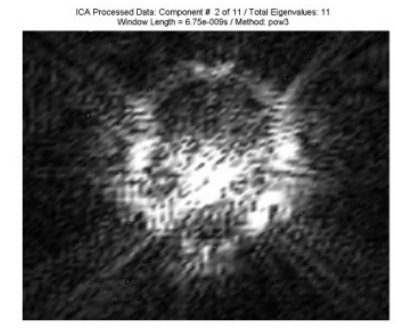

(b)

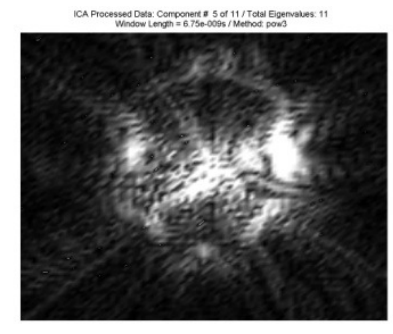

(e)

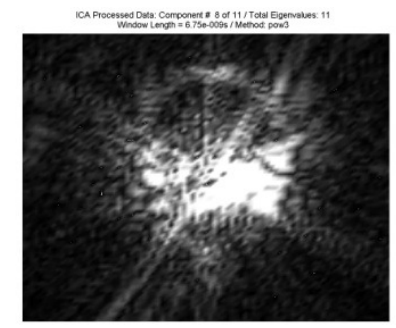

(h)

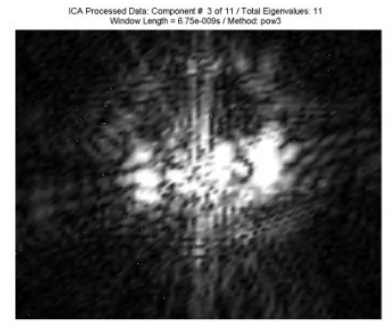

(c)

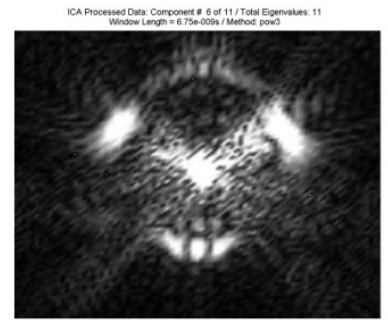

(f)

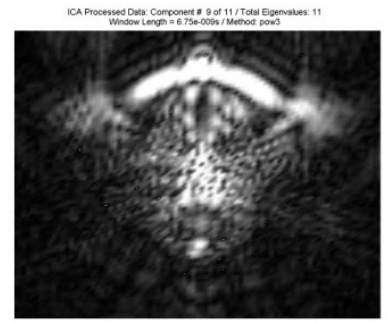

(i)

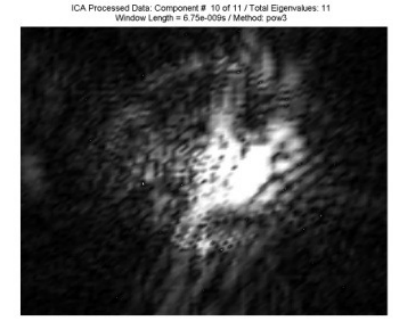

(j)

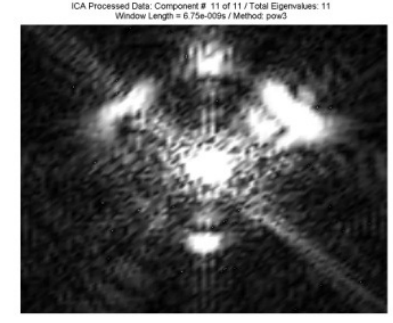

$(\mathrm{k})$

Figure 4-38 ICA results with $11 \mathrm{ICs}$ and $11 \mathrm{EVs}$ (a) component \#1 (b) component \#2 (c) component \#3 (d) component \#4 (e) component \#5 (f) component \#6 (g) component \#7 (h) component \#8 (i) component \#9 (j) component \#10 (k) component \#11

\subsubsection{Four Inch Sphere and Triangle}

The last target presented in this study is a 4" sphere suspended above a triangular pattern of cylindrical pipes. This target further increases the practicality of the target set. This target set is expected to include direct bounce scattering, creeping wave scattering and multi- 
bounce interaction scattering between the elements of the triangle as well as between the triangle and sphere.

An image of the target set can be seen in Figure 4-39 and the target's metric is shown in Figure. The results shown below will have the eigenvalues and the independent components both set to 6 . For this last target set results will be presented for each of the nonlinear estimators shown in Figure 2-4. The ISAR images of the original data and the time-gated data are presented in Figure 4-36.

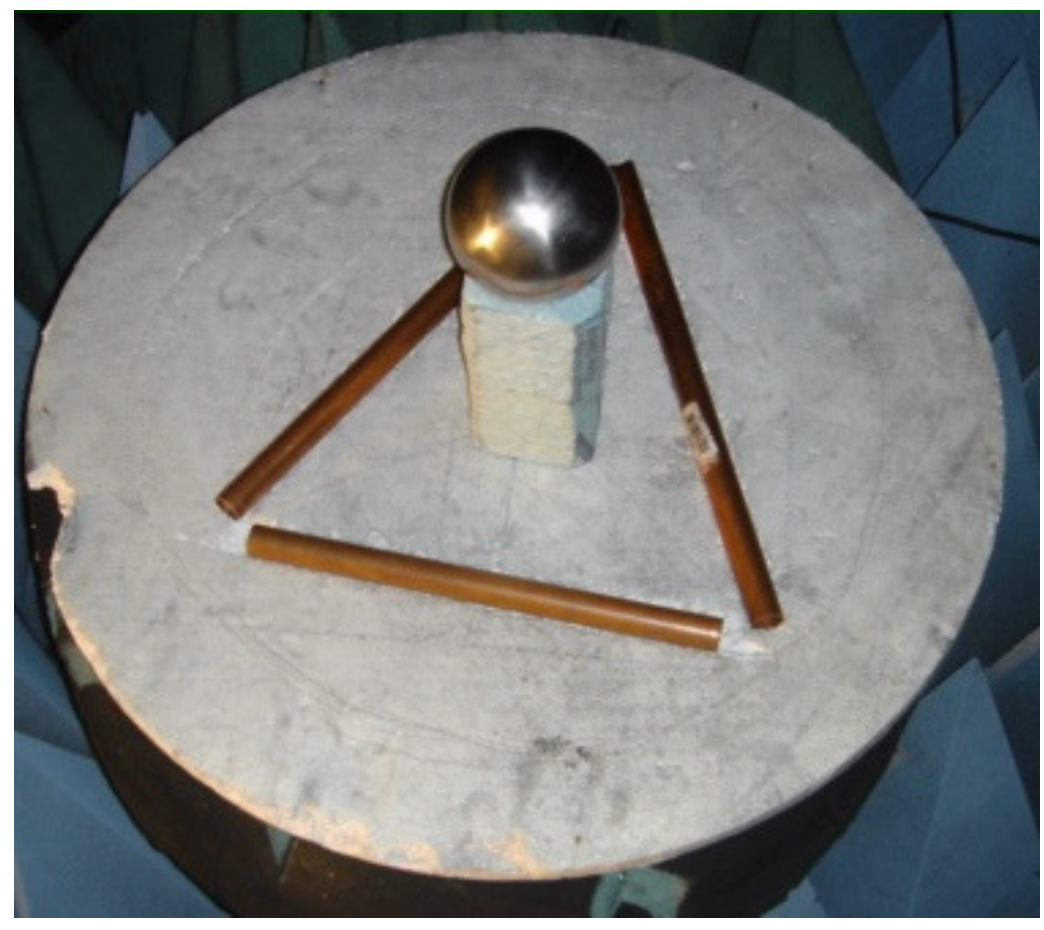

Figure 4-39 Photo of 4" sphere and triangle 


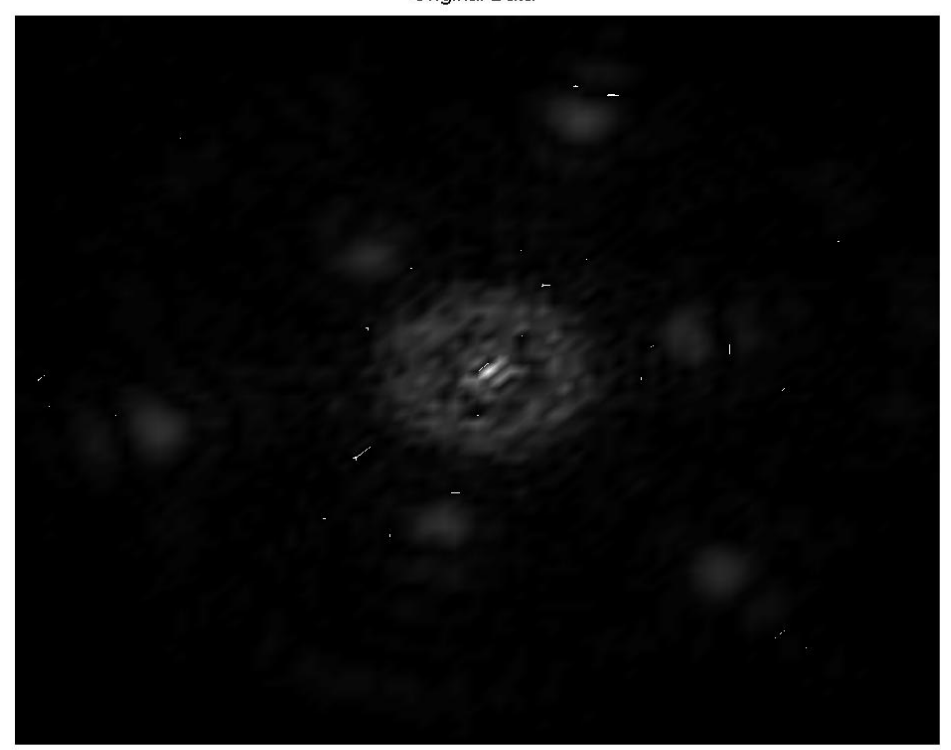

(a)

Time Windowed Data : Window Length $=6.75 \mathrm{e}-009 \mathrm{~s}$

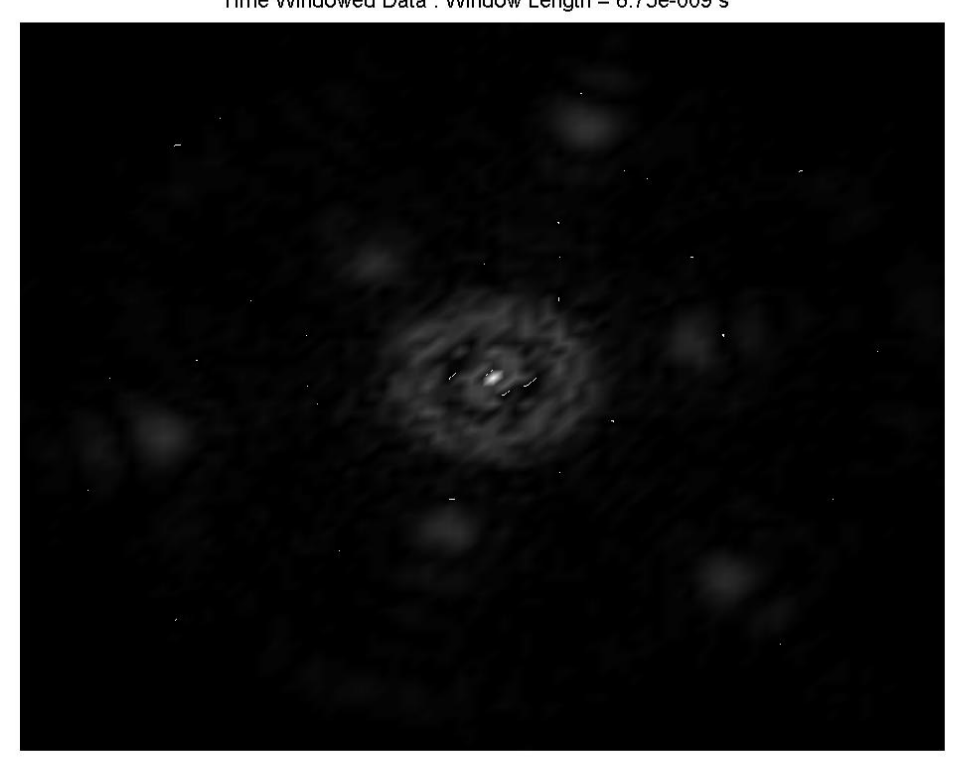

(b)

Figure 4-40 (a) ISAR image formed with raw scattering data (b) ISAR image formed from timegated scattering data 


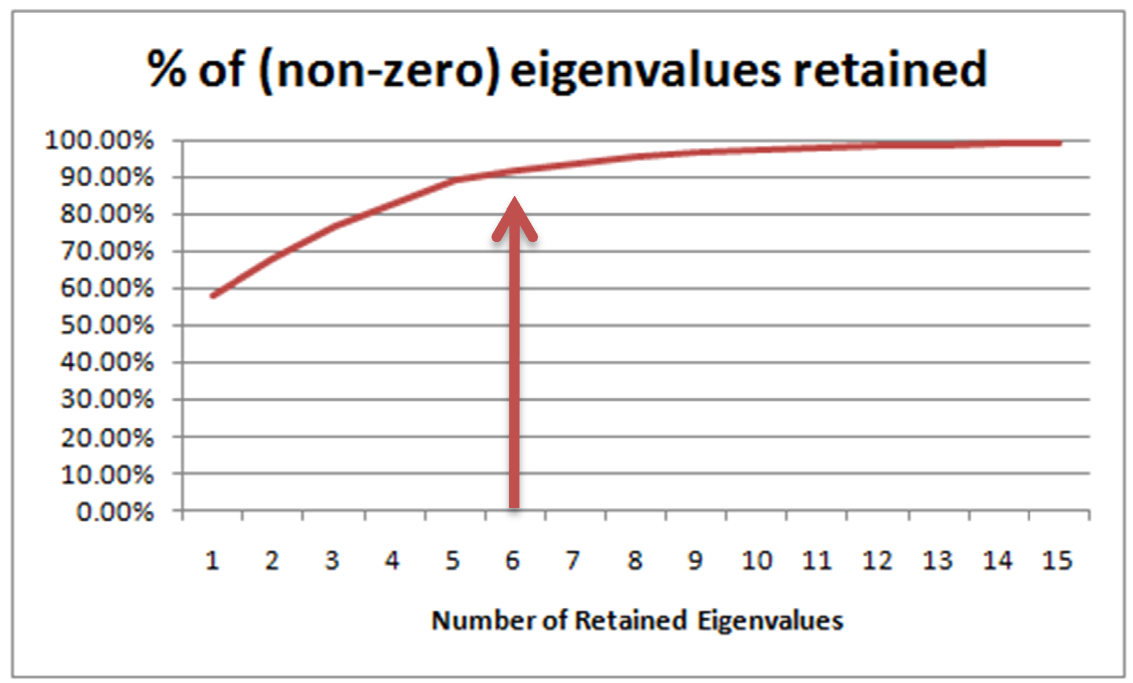

Figure 4-41 EV retention metric for 4" sphere and triangle 
ICA Processed Data: Component \# 1 of $6 /$ Total Eigenvalues: 6

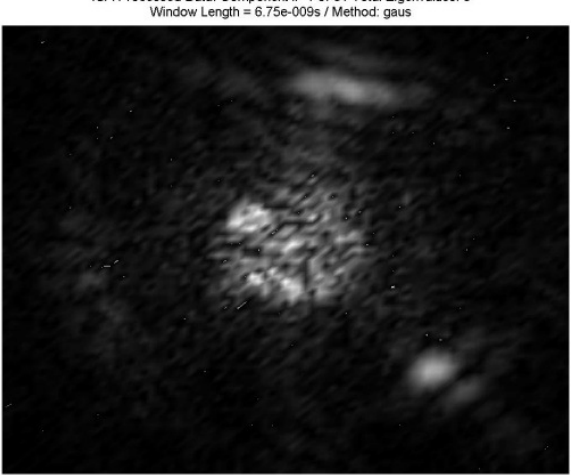

(a)

ICA Processed Data: Component \# 3 of 6 / Total Eigenvalues: 6
Window Lengoth $=6$.75e-0095/Method gaus

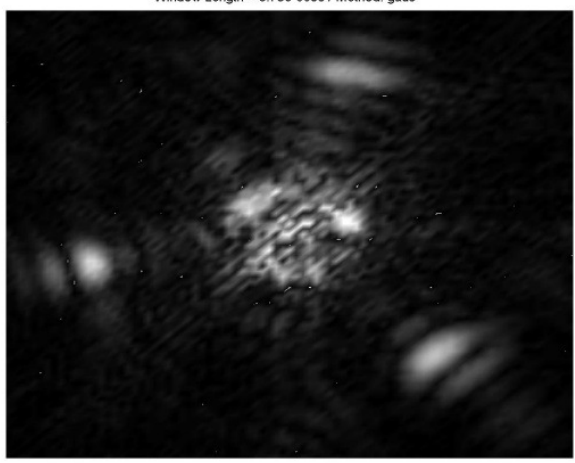

(c)

ICA Processed Data: Component \# 5 of 6 / Total Eigenvalues: 6

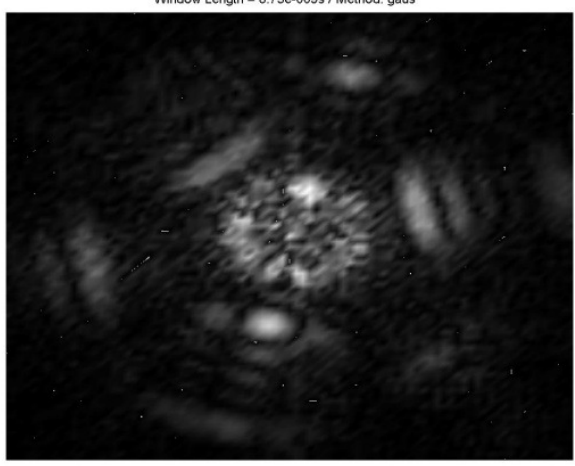

(e)
ICA Processed Data: Component \# 2 of $6 /$ Total Eigenvalues: 6
Window L Length $=6755$ - $609 \mathrm{~s} /$ Method gaus

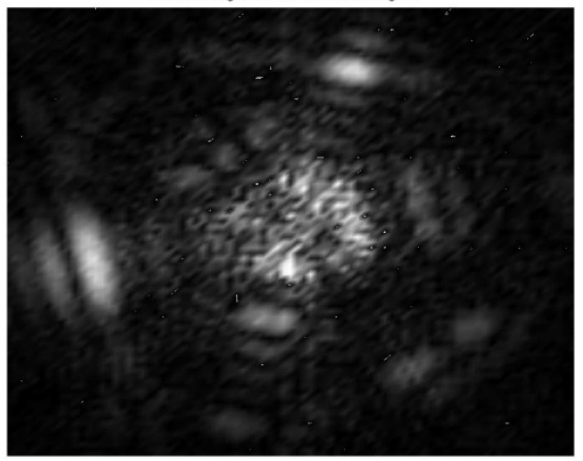

(b)

ICA Processed Data: Component \# 4 of $6 /$ Total Eigenvalues: 6
Window Length $=6.75$ - 009 s $/$ Method gaus

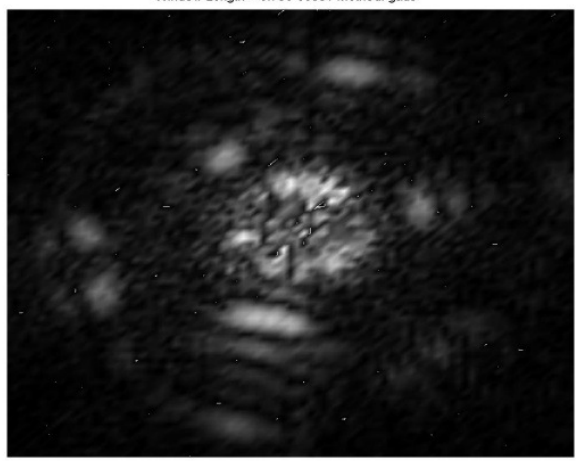

(d)

ICA Processed Data: Component \# 6 of $6 /$ Total Eigenvalues: 6
Window Length $=6.75$ - 009 s $/$ Methood gaus

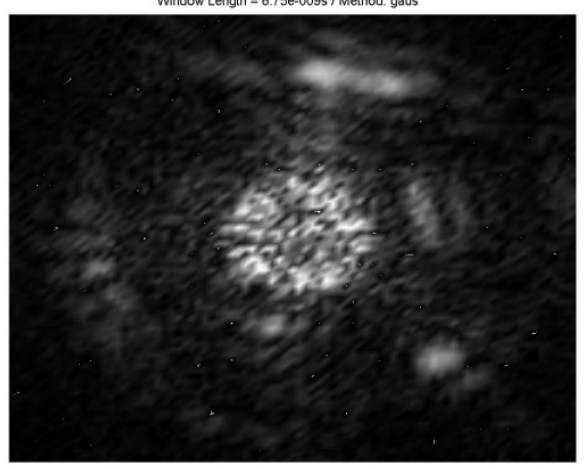

(f)

Figure 4-42 ICA results with 6 ICs and 6 EVs (a) gaus: component \#1 (b) gaus: component \#2 (c) gaus: component \#3 (d) gaus: component \#4 (e) gaus: component \#5 (f) gaus: component \#6 
ICA Processed Data: Component \# 1 of $6 /$ Total Eigenvalues: 6
Window Length $=675$ - 009 s $/$ Method pow 3

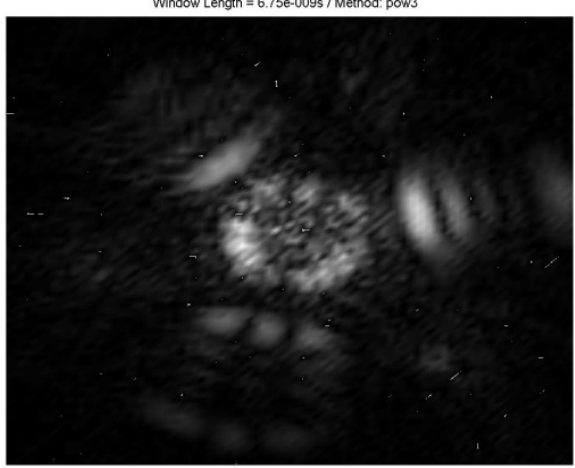

(a)

ICA Processed Data: Component \# 3 of 6 / Total Eigenvalues: 6
Window Lengoth $=6.75$ - 0095 / Method: pow 3

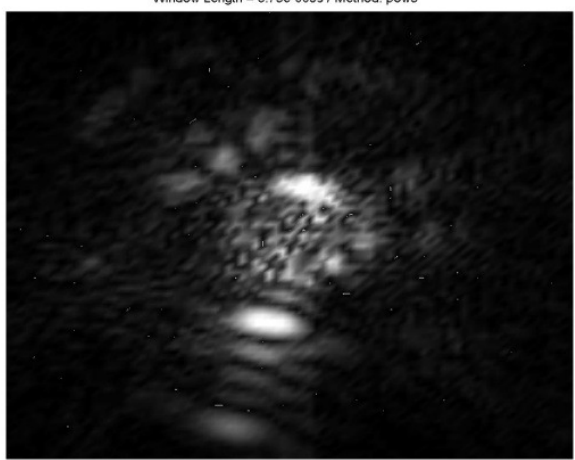

(c)

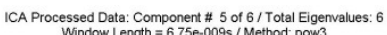

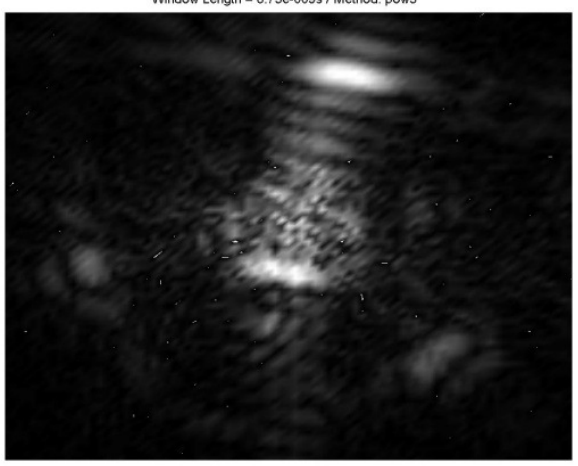

(e)
ICA Processed Data: Component \# 2 of $6 /$ Total Eigenvalues: 6
Window Length $=6.75 \mathrm{e}-009 \mathrm{~s} / \mathrm{Method.}$ pow 3

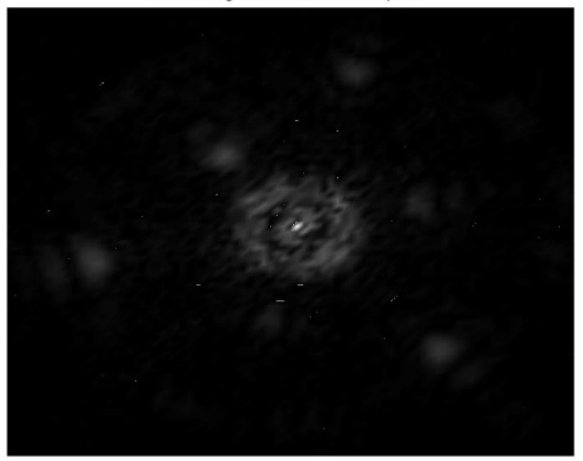

(b)

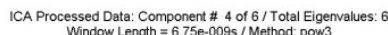

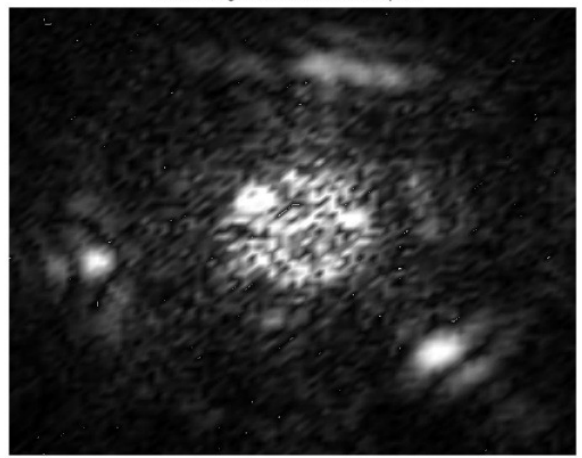

(d)

ICA Processed Data: Component \# 6 of $6 /$ Total Eigenvalues: 6
Window Length $=675$ - 609 s $/$ Method pow 3

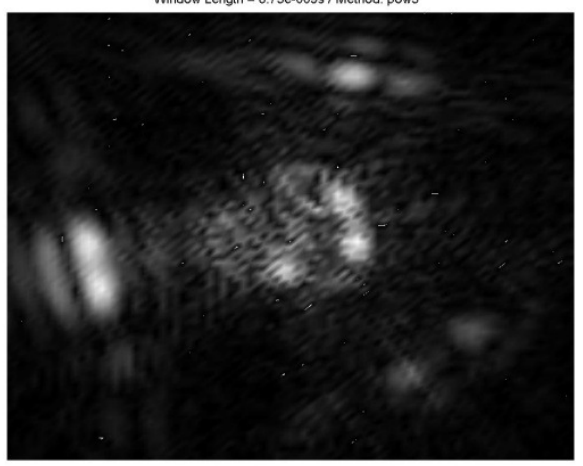

(f)

Figure 4-43 ICA results with 6 ICs and 6 EVs (a) pow3: component \#1 (b) pow3: component \#2 (c) pow3: component \#3 (d) pow3: component \#4 (e) pow3: component \#5 (f) pow3: component \#6 
ICA Processed Data: Component \# 1 of $6 /$ Total Eigenvalues: 6
Window Length $=6.75$ - $0095 /$ Method skew

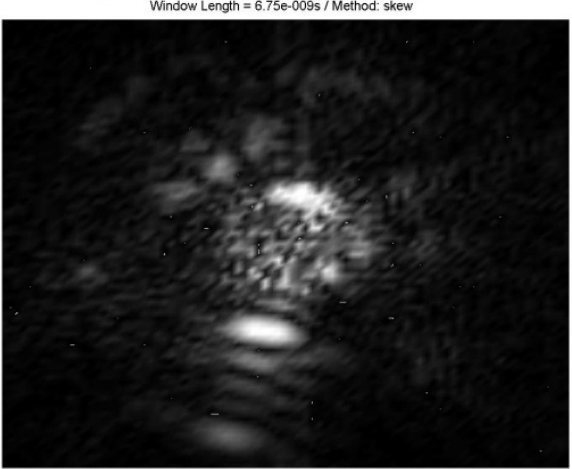

(a)

ICA Processed Data: Component \# 3 of $6 /$ Total Eigenvalues: 6
Window Lengoth $=6.75 \mathrm{se}-009 \mathrm{~s} /$ Method skew

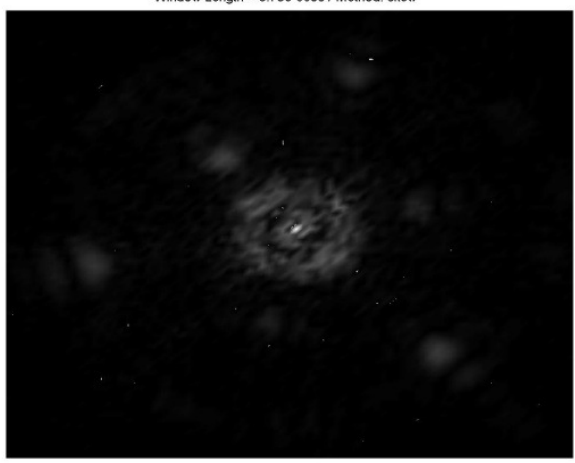

(c)

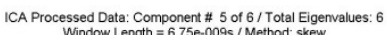

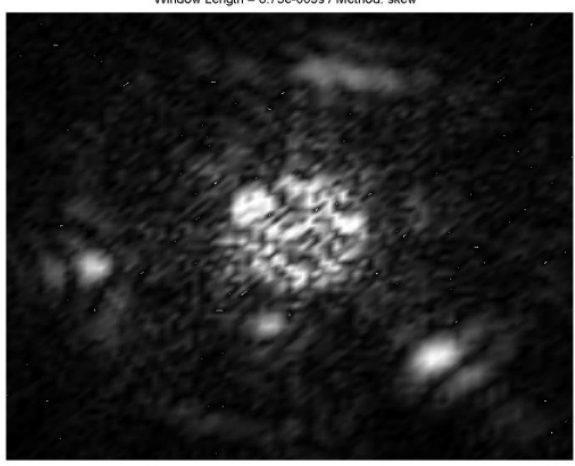

(e)
ICA Processed Data: Component \# 2 of $6 /$ Total Eigenvalues: 6
Window Length $=6.75 \mathrm{e}-009 \mathrm{~s} /$ Method: skew

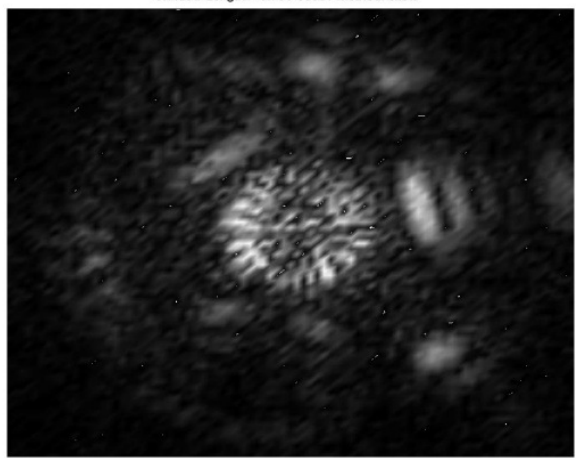

(b)

ICA Processed Data: Component \# 4 of $6 /$ Total Eigenvalues: 6
Window Lenoth $=6.75$ - 009 s $/$ Method: skew

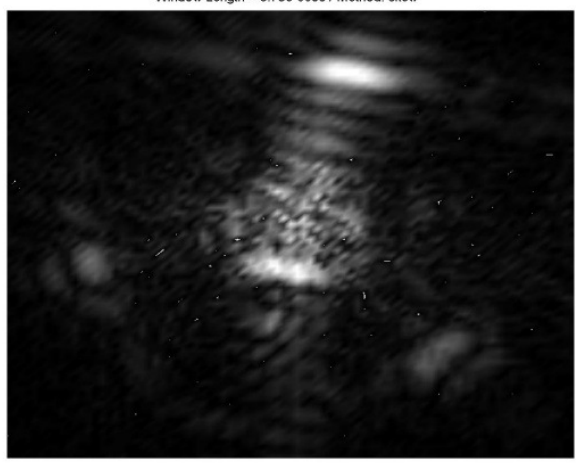

(d)

ICA Processed Data: Component \# 6 of $6 /$ Total Eigenvalues: 6
Window Length $=6.75$ - 009 s $/$ Methood skew

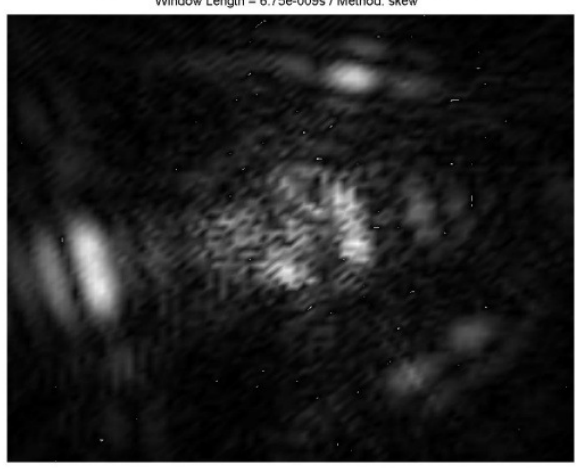

(f)

Figure 4-44 ICA results with 6 ICs and 6 EVs (a) skew: component \#1 (b) skew: component \#2 (c) skew: component \#3 (d) skew: component \#4 (e) skew: component \#5 (f) skew: component \#6 
ICA Processed Data: Component \# 1 of $6 /$ Total Eigenvalues: 6
Window Length $=6.75 \mathrm{e}-009 \mathrm{~s} /$ Method tanh

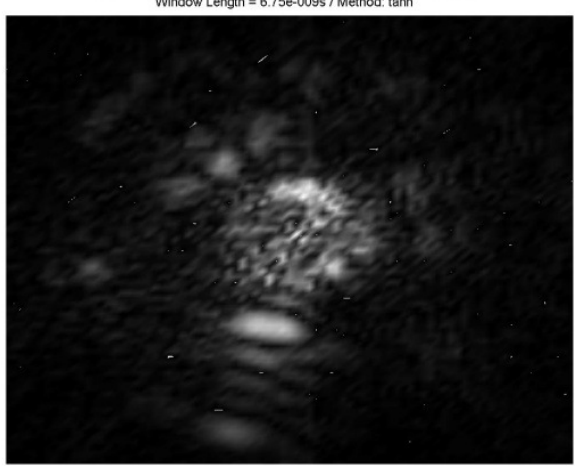

(a)

ICA Processed Data: Component \# 3 of 6 / Total Eigenvalues: 6
Window Length $=675$ - 6095 / Method: tanh

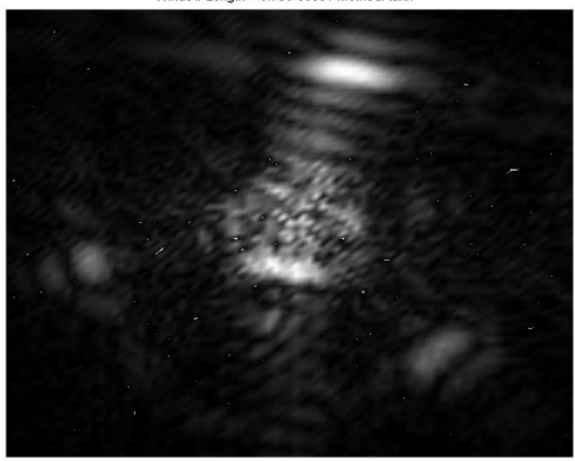

(c)

ICA Processed Data: Component \# 5 of 6 / Total Eigenvalues: 6

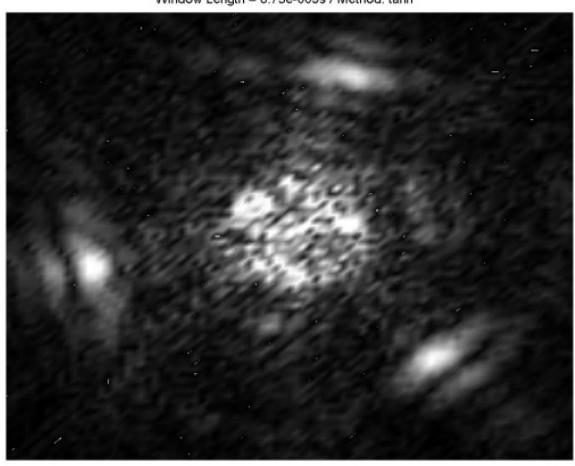

(e)
ICA Processed Data: Component \# 2 of $6 /$ Total Eigenvalues: 6
Window Length $=6.75$ - $009 \mathrm{~s} /$ Method: tanh

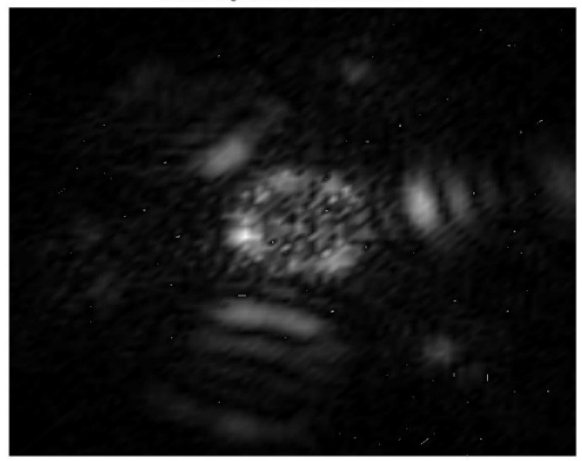

(b)

ICA Processed Data: Component \# 4 of $6 /$ Total Eigenvalues: 6
Window Length $=6.75$ - 009 s $/$ Method tanh

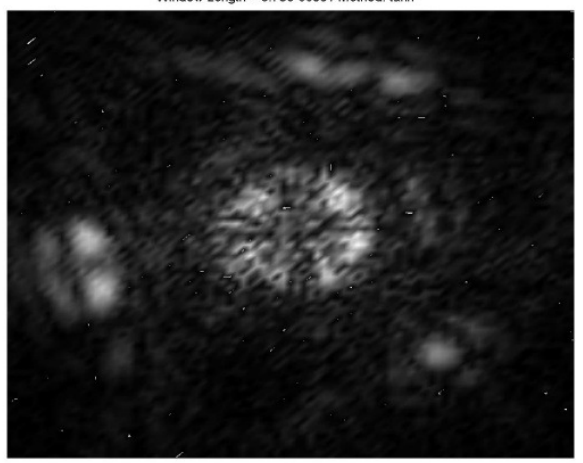

(d)

ICA Processed Data: Component \# 6 of $6 /$ Total Eigenvalues: 6
Window Length $=6.75$ - 009 s $/$ Methood tanh

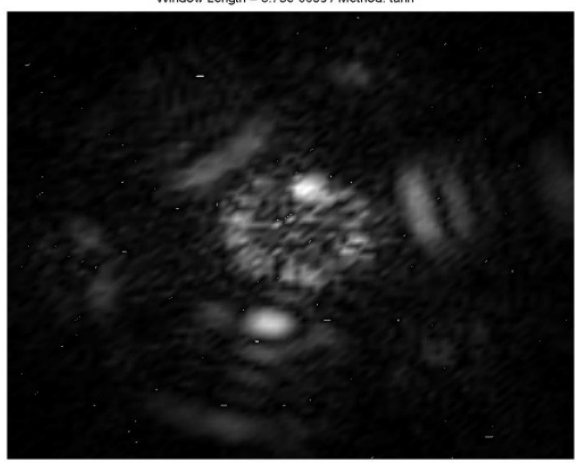

(f)

Figure 4-45 ICA results with 6 ICs and 6 EVs (a) tanh: component \#1 (b) tanh: component \#2 (c) tanh: component \#3 (d) tanh: component \#4 (e) tanh: component \#5 (f) tanh: component \#6 


\section{CHAPTER 5}

\section{ANALYSIS}

The scattering mechanisms of a target can be determined by the various geometrical features of the target. For example a singular sphere has two scattering mechanisms. One of the mechanisms is the specular scatter from the surface of the target normal to the radar antenna and the other is the creeping wave. It is known that the magnitude of the specular return is 10 s of dBs greater than that from the creeping wave [19]. For this reason, the specular scattering mechanism is expected to be highly dominant in for the case of a single sphere. Evidence of this can be seen in the following two sets of figures that the direct bounce scattering is included in every independent component extracted. Figure 5-1 shows the nine independent components of a single 6" sphere. All of the images formed based on the extracted independent components contain a fairly well defined circular shape which corresponds to the circumference of the sphere. 


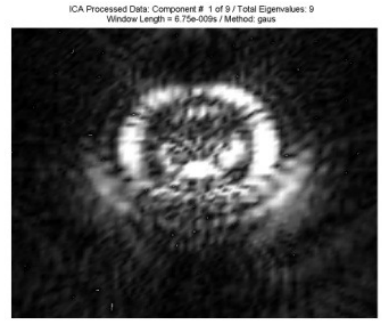

(a)

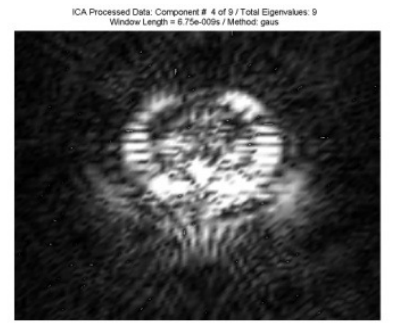

(d)

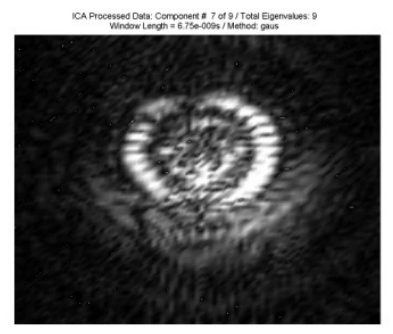

(g)

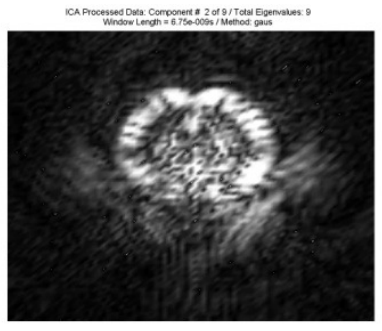

(b)

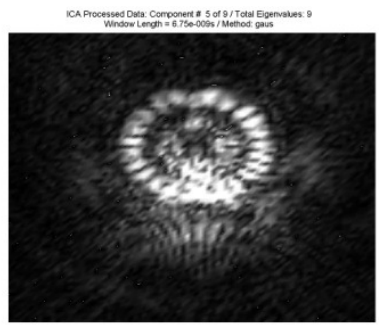

(e)

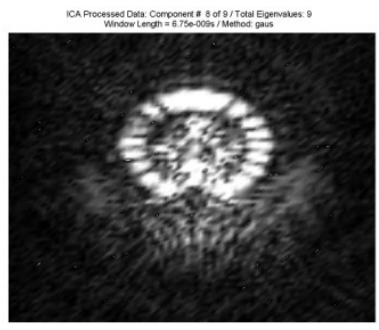

(h)

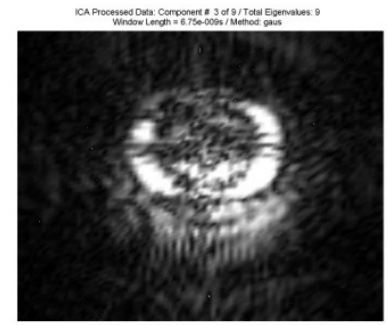

(c)

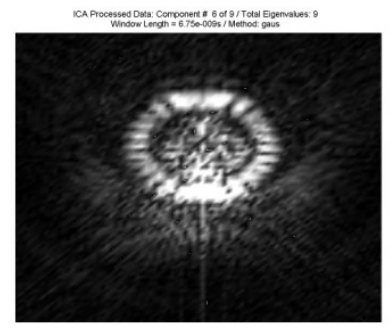

(f)

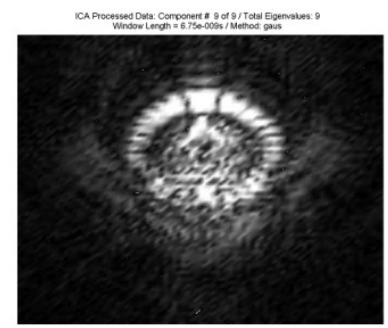

(i)

Figure 5-1 Independent components for 6" sphere (a) component \#1 (b) component \#2 (c) component \#3 (d) component \#4 (e) component \#5 (f) component \#6 (g) component \#7 (h) component \#8 (i) component \#9

Figure 5-2 shows the eight components extracted for a single 4" sphere. Again, all of the images contain a fairly well defined circular shape which corresponds to the specular echo created by the normal surface of the sphere. 


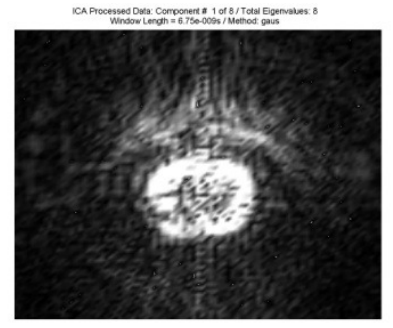

(a)

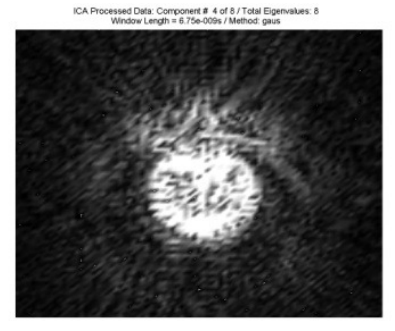

(d)

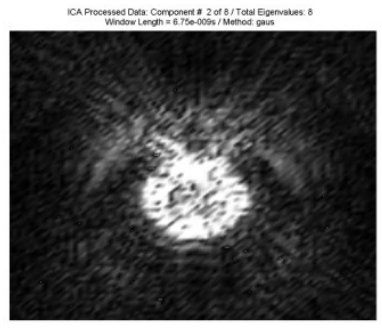

(b)

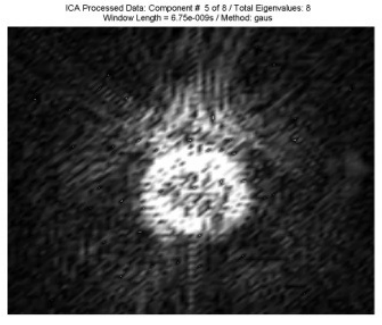

(e)

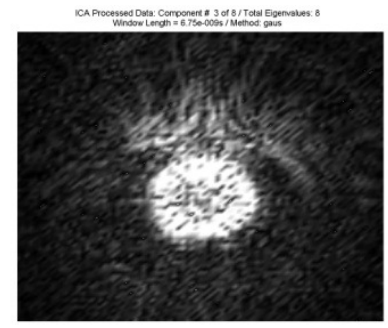

(c)

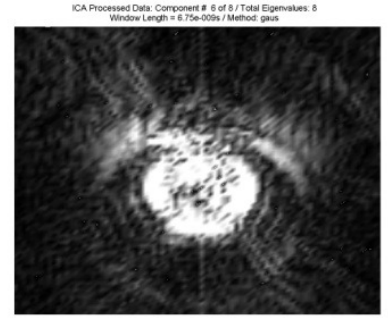

(f)

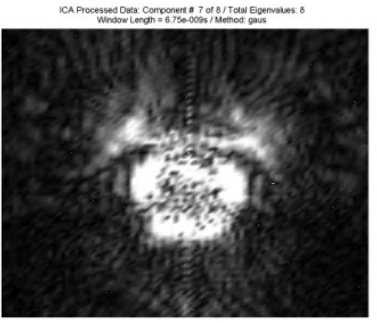

(g)

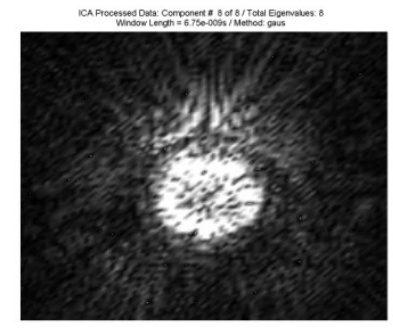

(h)

Figure 5-2 Independent components for 4" sphere (a) component \#1 (b) component \#2 (c) component \#3 (d) component \#4 (e) component \#5 (f) component \#6 (g) component \#7 (h) component \#8

A much more interesting case is created when a target's geometry allows for a return, which is a product of multiple reflections, to be presented to the sensing system. The magnitude of the multiple path return is expected to be closer to that of the direct bounce than the creeping wave [19]. The first example of a multiple bounce scattering geometry is that of the 6" and 4" spheres spaced at 8". 


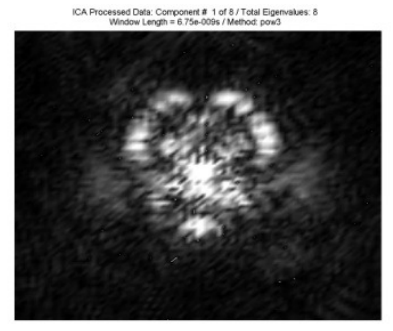

(a)

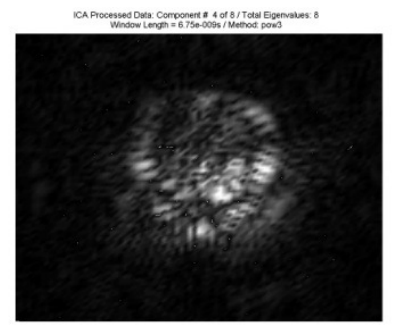

(d)

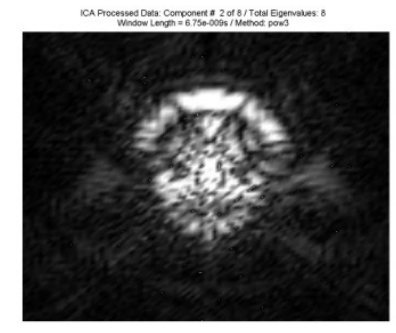

(b)

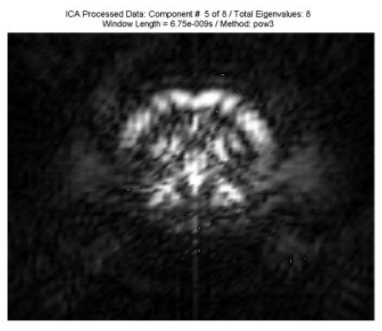

(e)

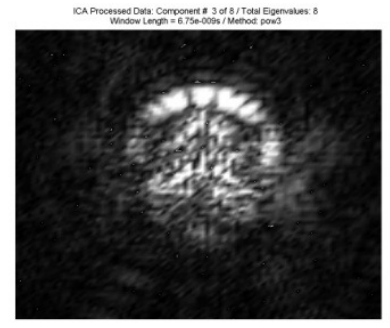

(c)

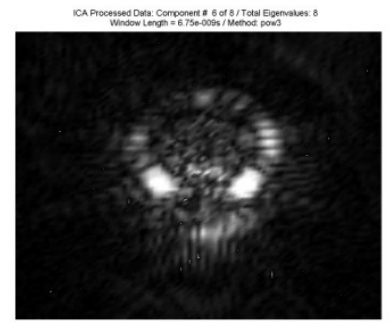

(f)

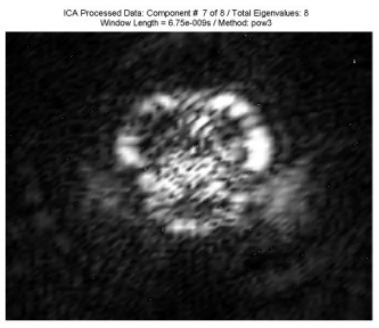

(g)

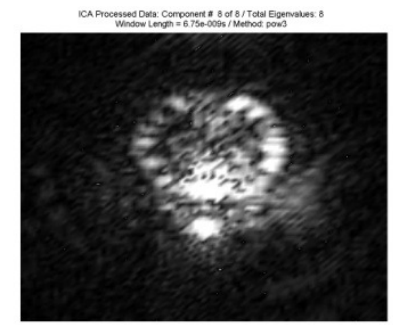

(h)

Figure 5-3 Independent components for 6" and 4" spheres with 8" spacing (a) component \#1 (b) component \#2 (c) component \#3 (d) component \#4 (e) component \#5 (f) component \#6 (g) component \#7 (h) component \#8

Given the results from the two spheres in isolation it is expected that one or more of the extracted components would be the direct bounce returns from the spheres. Figure 5-3(b) shows a good example of the surface return for both of the spheres in the target set. The utility of the application of ICA to ISAR data can be seen in Figure 5-3(f). This figure shows how this extracted component has emphasized the region between the spheres in which one would expect multiple bounce reflections to occur.

Another example, which shows how ICA can be used to extract scattering mechanisms which are independent from the direct bounce return, is given by the same target set at a greater separation. Figure 5-4 shows the 11 independent components associated with the 6" sphere and the 4" sphere at 16 " of separation. An unexpected result shown by these images is that the direct bounce return of the two spheres is no longer a dominating element within the components. This 
is evidenced by the absence of the clearly defined circular shapes present in the images of the previous cases. The independent components extracted for this target set appear to be more closely related to multiple bounce and creeping wave effects. As in the prior data set Figure 5-4(c) and Figure 5-4(h) show images in which emphasis is placed the region where multiple path interaction is expected. Figure 5-4(e), Figure 5-4(f) and Figure 5-4(g) illustrate multiple bounce cases of associated with progressively shallower angles of incidence. This is shown by the spatial displacement of the multiple bounce returns which is signatory of additional path delay associated with the more obtuse dihedral geometry. Finally Figure 5-4(a), Figure 5-4(d) and Figure 5-4(i) show emphasis being placed on portions of the image which are characteristic of a creeping wave return. 


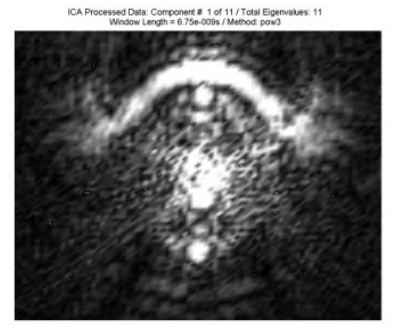

(a)

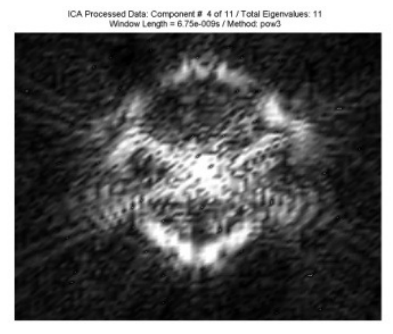

(d)

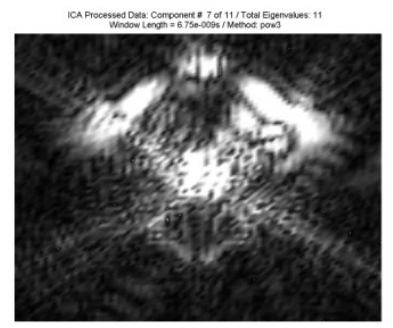

(g)

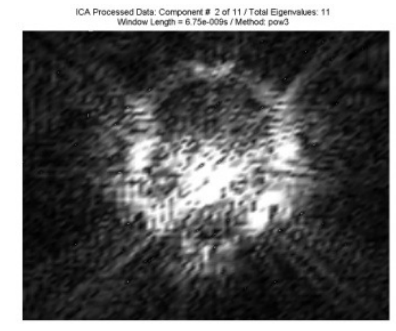

(b)

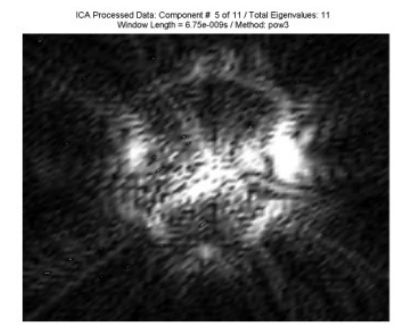

(e)

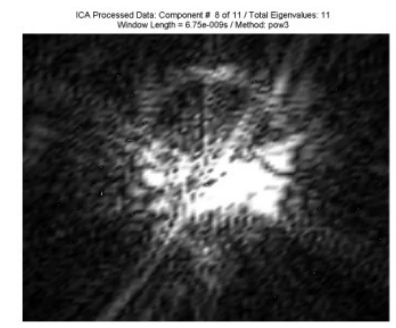

(h)

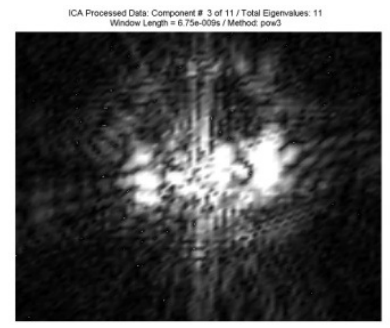

(c)

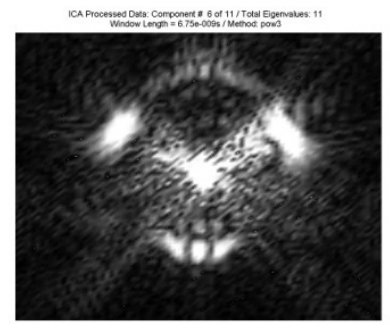

(f)

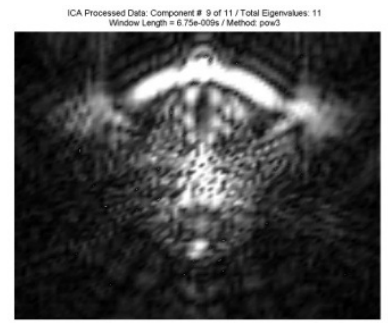

(i)

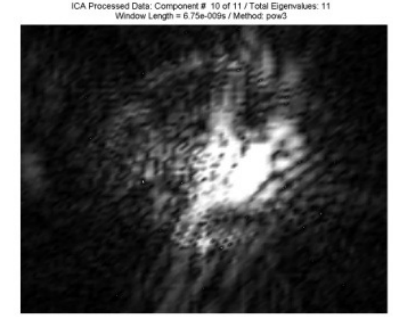

(j)

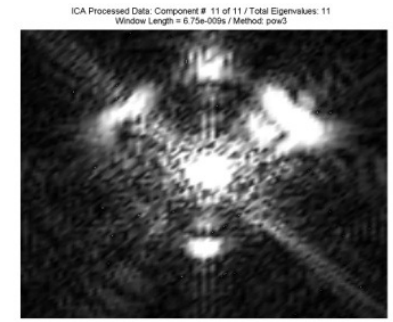

(k)

Figure 5-4 Independent components for 6" and 4" spheres with 16" spacing (a) component \#1 (b) component \#2 (c) component \#3 (d) component \#4 (e) component \#5 (f) component \#6 (g) component \#7 (h) component \#8 (i) component \#9 (j) component \#10 (k) component \#11

The last case presented is by far the most complex due to the multitude of scattering elements and multiple bounce return paths. The images below each contain a well-defined circular image which represents the direct bounce return of the sphere. This indicates that the direct bounce return from the sphere is an integral element of the extracted components for this target set. Also in this case, a creeping wave associated with the broadside of the cylinders can 
be seen to be emphasized in Figure 5-5(a), Figure 5-5(b) and Figure 5-5(f). The characteristic periodic echo structure typically associated with a creeping wave along a small diameter object can be seen. This phenomenon occurs as a result of the creeping wave shedding less energy along the short circumferential distance and therefore the energy associated with multiple traverses of the periphery of the object can be detected. The other three components represented by Figure 5-5(c), Figure 5-5(d) and Figure 5-5(e), show emphasis being placed on the multipath region related to the intersection of the cylindrical rods. It can be seen that the most emphasized portion of the image also contains the periodic signature of varying angles of incidence on the dihedral geometry. 
ICA Processed Data: Component \# 1 of $6 /$ Total Eigenvalues: 6

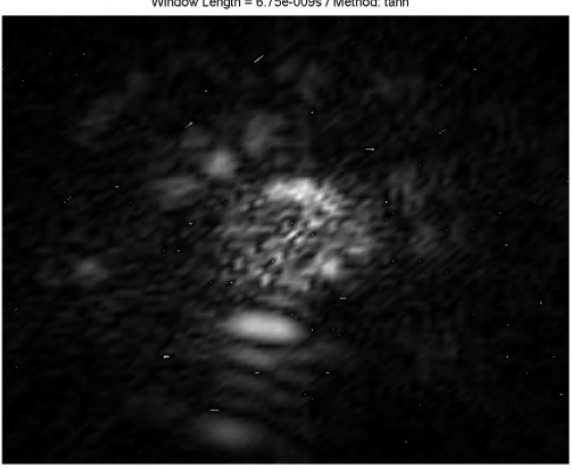

(a)

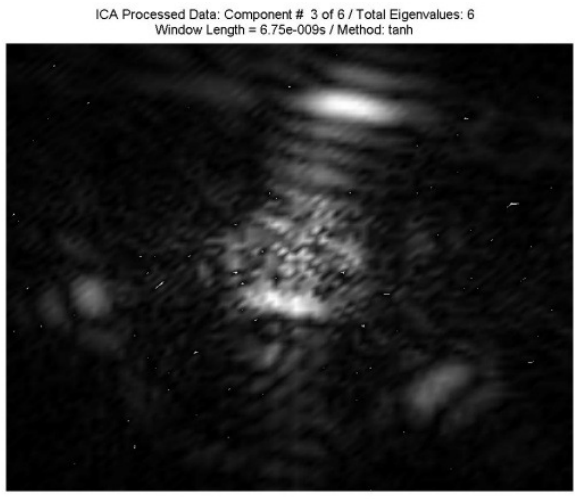

(c)

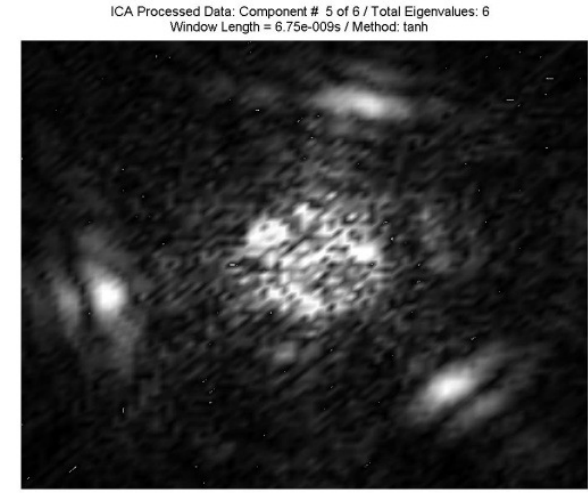

(e)

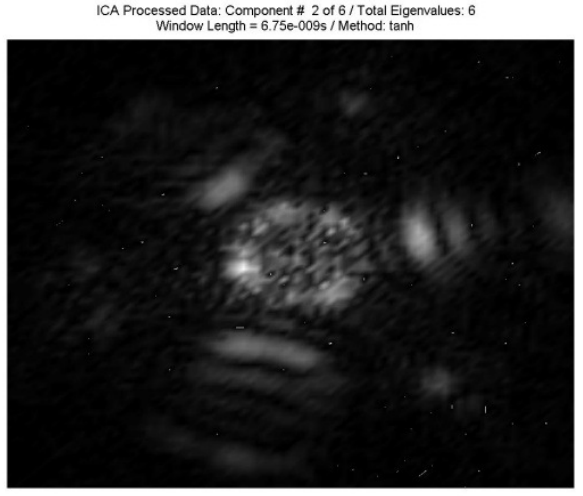

(b)

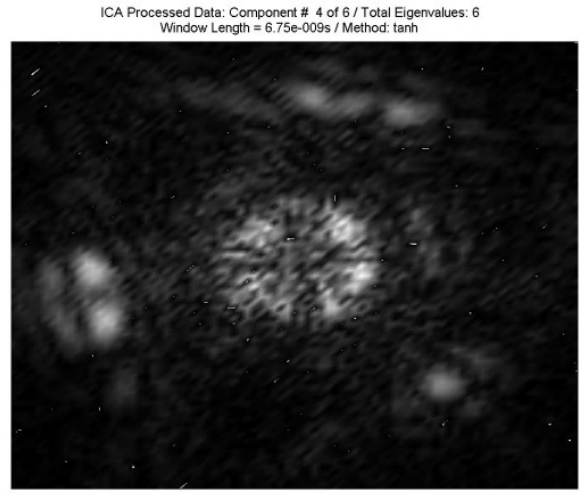

(d)

ICA Processed Data: Component \# 6 of $6 /$ Total Eigenvalues: 6
Window Length $=6.75$ - 009 s $/$ Method: tanh

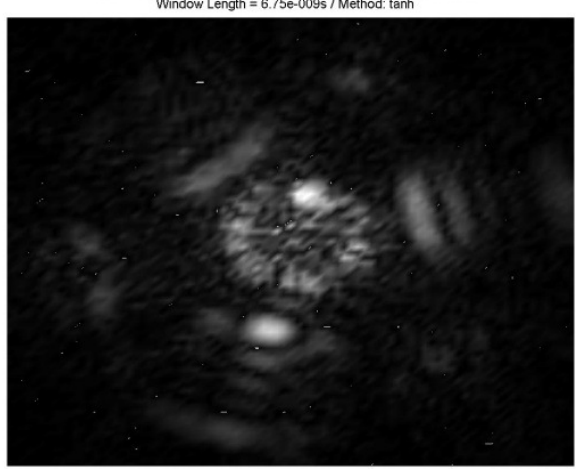

(f)

Figure 5-5 Independent components for 4" spheres and triangle (a) tanh: component \#1 (b) tanh: component \#2 (c) tanh: component \#3 (d) tanh: component \#4 (e) tanh: component \#5 (f) tanh: component \#6 


\section{CHAPTER 6}

\section{CONCLUSIONS}

This thesis has presented an overview of inverse synthetic aperture radar (ISAR) processing, an overview of independent component analysis and the methodology for the marriage of the two.

Physical optics based ISAR processing is subject to the assumptions made in the physical optic approximations. The key assumptions are that the fields on the non-illuminated portion of the target are zero, the target is being observed in the far-field, and that the curvature of the target is much greater than a wavelength. Another key assumption made in the use of Bojarski's identity is that target is strictly convex. These assumptions do not accurately reflect reality when considering the scattering mechanisms such as traveling waves interacting with edges and seams, creeping waves and multi-bounce interaction.

As an enhancement to the physical optics based ISAR techniques the use of independent component analysis (ICA) has been explored. ICA is a well known method for uncovering hidden elements in data mixtures. This thesis has shown that application of independent component analysis to ISAR data is capable of emphasizing or picking out embedded scattering elements from the data mixture.

Considering radar measurements made in the ISAR configuration as mixtures of the various scattering mechanisms on a target, it is possible to extract independent components which are related to the scatterers. 
Analysis of the results presented above show that there is correlation between scatterers and extracted independent components. The proof of this lies in the fact that the extracted independent components shown above emphasize different portions of the ISAR image.

This thesis is presenting the framework for application of ICA to ISAR and has given some results showing the promise of this application. 


\section{REFERENCES}

[1] W. G. Carrara, R. S. Goodman, and R. M. Majewski, Spotlight Synthetic Aperture Radar: Signal Processing Algorithms. Norwood, MA, USA: Artech House, 1995.

[2] M. I. Skolnik, Ed., Radar Handbook, 3rd ed. Chicago: McGraw-Hill, 2008.

[3] M. Cheney and B. Borden, "Microlocal analysis of GTD-based SAR models," in Algorithms for Synthetic Aperture Radar Imagery XII, vol. 5808, 2005, p. 15-23.

[4] M. Ferrara, M. Cheney, and G. Arnold, "Identification of Multiple-Scattering Events from 3D GTD-Based Parametric Scattering Models," in Radar Sensor Technology XI, vol. 6547, Orlando, FL, USA, 2007, p. 65470M.

[5] D. A. Garren, J. S. Goldstein, D. R. Obuchon, R. R. Greene, and J. A. North, "SAR image formation algorithm with multipath reflectivity estimation," in Radar Conference, Philadelphia, PA, 2004, pp. 323-328.

[6] Y. Shen, "Dynamic Microwave Imaging," PhD Dissertation, University of Pennslyvania, 1987.

[7] J. Bredow, K. Xie, R. Porco, and M. Shah, "An Experimental Study on the Use of Multistatic Imaging For Investigating Electromagnetic Wave-object Interaction," Journal of Electronmagnetic Waves and Applications, vol. 7, no. 6, pp. 811-831, 1993.

[8] N. N. Bojarski, "A survey of physical optics inverse scattering identity," IEEE Transactions on Antennas \& Propagation, vol. AP-30, no. 5, pp. 980-989, Sep. 1982.

[9] H. J. Li, N. H. Farhat, Y. Shen, and C. L. Werner, "Image Understanding and Interpretation in Microwave Diversity Imaging," IEEE Transactions on Antennas and Propagation, vol. 37, no. 8, pp. 1048-1057, 1989.

[10] M. Cheney and R. J. Bonneau, "Imaging that exploits multipath scattering from point scatterers," Inverse Problems, vol. 20, pp. 1691-1711, 2004.

[11] J. V. Stone, Independent Component Analysis: A Tutorial Introduction. Cambridge, MA, United States of America: MIT Press,, 2004.

[12] P. Comon, "Independent component analysis, a new concept?," Signal Processing, vol. 36, no. 3, pp. 287-314, 1994.

[13] A. Hyvärinen, "Fast and robust fixed-point algorithms for independent component analysis," IEEE Transactions on Neural Networks, vol. 10, pp. 626-634, Mar. 1999.

[14] A. Hyvärinen, J. Karhunen, and E. Oja, Independent Component Analysis. New York, United States of America: John Wiley \& Sons, Inc., 2001.

[15] M. Potter and W. Kinsner, "Signal separation by independent component analysis and fuzzy estimators," in IEEE Annual Meeting of the Fuzzy Information Processing Society, BANFF, ABERTA, CANADA, 2004, pp. 838-843. 
[16] F. T. Ulaby and M. C. Dobson, Handbook of Radar Scattering Statistics for Terrain. Norwood, MA, United States of America: Artech House, 1989.

[17] ICA Central. [Online]. http://www.tsi.enst.fr/icacentral/index.html

[18] B. D. Jersak, "Time domain analysis of measured frequency domain radar cross-section data," MS Thesis, University of Texas at Arlington, Arlington, 1988.

[19] E. F. Knott, J. F. Shaeffer, and M. T. Tuley, Radar Cross Section, 2nd ed. Raleigh, United States of America: SciTech Publishing, 2004.

[20] M. Cheney and B. Borden, "Microlocal structure of inverse synthetic aperture radar data," Inverse Problems, vol. 19, pp. 173-193, 2009. 


\section{BIOGRAPHICAL INFORMATION}

Jeffrey Hall graduated from the University of Texas at San Antonio in 2002 with a Bachelor of Science in Electrical Engineering. He is currently pursuing a Master of Science in Electrical Engineering at the University of Texas at Arlington, expected graduation August, 2009. He has worked for Lockheed Martin since 2002. He is currently with the Missiles and Fire Control unit of the Electronic Systems division in Grand Prairie, Texas. Current research interests include radar imaging, radar cross section measurements, antennas and signal processing and radar system design. 